\title{
Liberalismo, democrazia, socialismo
}

\section{L'itinerario di Carlo Rosselli}

\section{Hewentwis,

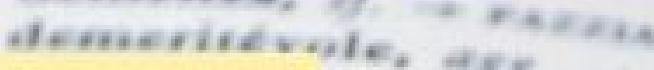

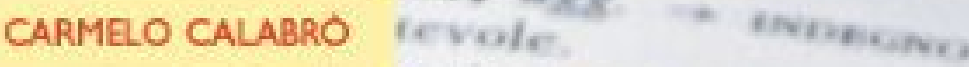

eorner. mitrito.

deminiso, sm, artefíc democratico, agg

comer. aristocratico || depolare || estenseatore, $\rightarrow$ democrazia, sf. governomgogico. momencl. (vari tipi) del lavovranit avanzata, radicale, libelavoro, popolpopolare, moderata, cristiana, orite, socialistare, progr. werth. democratizzare comer. autovrazia. rir

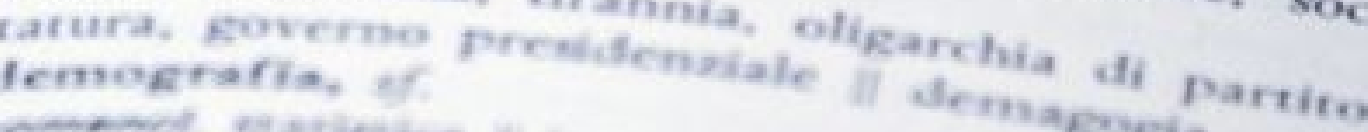

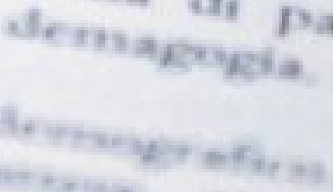


STRUMENTI

PER LA DIDATTICA E LA RICERCA

$-87-$ 


\section{Carmelo Calabrò}

\section{Liberalismo, democrazia, socialismo}

L'itinerario di Carlo Rosselli 


\section{METHEXIS}

\section{Comitato Scientifico}

Brunella Casalini (Direttore, Università di Firenze) Maria Chiara Pievatolo (Direttore, Università di Pisa) Nico De Federicis (Università di Pisa) Roberto Gatti (Università di Perugia) Roberto Giannetti (Università di Pisa) Michele Nicoletti (Università di Trento) Claudio Palazzolo (Univeristà di Pisa) Gianluigi Palombella (Università di Parma) Salvatore Veca (Università di Pavia) Danilo Zolo (Università di Firenze)

Volumi pubblicati

Calabrò C., Liberalismo, democrazia, socialismo Costantini D. (a cura di), Multiculturalismo alla francese? 
Liberalismo, democrazia, socialismo : l'itinerario di Carlo Rosselli / Carmelo Calabrò. - Firenze : Firenze University Press, 2009.

(Strumenti per la didattica e la ricerca ; 87)

ISBN 978-88-6453-084-0 (print)

ISBN 978-88-6453-086-4 (online)

Questo volume è pubblicato con il contributo dell'Università degli Studi di Pisa

Progetto grafico di Alberto Pizarro Fernández

Edizione digitale:

2009 Creative Commons

(C) 2009 Firenze University Press

Università degli Studi di Firenze

Firenze University Press

Borgo Albizi, 28, 50122 Firenze, Italy

http://www.fupress.com/

Printed in Italy 
Ad Antonio e Giulia 

CAPITOLO PRIMO

LA FORMAZIONE GIOVANILE 5

1. L'ambiente familiare 5

2. L'esperienza della guerra 10

3. Studi sul sindacalismo 19

CAPITOLO SECONDO

I CONTI CON IL LIBERALISMO $\quad 27$

1. Liberalismo e liberismo 27

2. Il confronto con Einaudi 33

3. Liberalismo socialista 37

CAPITOLO TERZO

UNA PROPOSTA SOCIALISTA $\quad 49$

1. Socialismo e marxismo 49

2. Il rapporto con il socialismo inglese 58

3. «Il Quarto Stato»: l'unità nel segno di un socialismo
democratico

CAPITOLO QUARTO

SOCIALISMO LIBERALE $\quad 81$

1. Marxismo e revisionismo 81

2. Dal revisionismo al socialismo liberale 86

3. Socialismo come realizzazione del liberalismo 92

4. La democrazia tra libertà e giustizia possibile 100

$\begin{array}{ll}\text { CONCLUSIONI } & 105\end{array}$

$\begin{array}{ll}\text { INDICE DEI NOMI } & 107\end{array}$ 



\section{INTRODUZIONE}

Rosselli liberale? Rosselli democratico? Rosselli socialista? L'ordine delle domande rispecchia quello dei termini che compongono il titolo di questo libro. Ma forse è più proficuo chiedersi: quanto e in che modo liberale, democratico, socialista? Alla luce degli orientamenti interpretativi (vecchi e nuovi), l'interrogativo non sembra del tutto ozioso. La letteratura critica ha moltiplicato le risposte, con formule combinatorie differenti e non sempre compatibili. Rosselli socialista liberale (classica e coerente con l'autodefinizione); Rosselli socialista democratico (non socialdemocratico $)^{1}$; Rosselli integralmente democratico ${ }^{2}$; Rosselli sicuramente socialista, tendenzialmente democratico, discutibilmente liberale ${ }^{3}$.

Non è mia ambizione individuare definizioni nuove e aggiuntive rispetto a un lungo, articolato e contrastato dibattito di storia delle idee tuttora in corso e particolarmente vivace negli ultimi anni. Né la scelta di partire dal liberalismo per arrivare al socialismo, via democrazia, allude alla convinzione di un percorso evolutivo lineare e omogeneo che conduce Rosselli da posizioni tout court liberali a posizioni tout court socialiste. L'intenzione sarebbe qui piuttosto quella di condurre l'analisi degli scritti del futuro leader di GL all'interno di un paradigma contestualista, per tentare di conferire maggiore chiarezza ai concetti tramite l'ancoraggio a una dimensione storicamente definita. E questo non per negare la potenziale attualità delle idee di Rosselli, ma per evitare la distorsione interpretativa che può derivare da operazioni forzatamente attualizzanti.

Per affrontare in modo corretto e "concreto" il pensiero di Rosselli, risulta pertanto necessario tenere conto delle declinazioni che i termini liberalismo, democrazia e socialismo assumono nell'ambito del discorso teorico-politico durante gli anni della crisi europea, tra la prima guerra 2002.

${ }^{1}$ Cfr. P. Bagnoli, Carlo Rosselli. Il socialismo della libertà, Polistampa, Firenze

${ }^{2}$ Di «conflittualismo democratico» ha parlato F. Sbarberi, L'utopia della libertà eguale, Bollati Boringhieri, Torino1999, p. 69.

${ }^{3}$ Cfr. G. Bedeschi, La fabbrica delle ideologie. Il pensiero politico nell'Italia del Novecento, Laterza, Roma-Bari 2002, pp. 295-305.

Carmelo Calabrò, Liberalismo, democrazia, socialismo. L'itinerario di Carlo Rosselli, ISBN 978-88-6453-084-0 (print), ISBN 978-88-6453-086-4 (online)

(c) 2009 Firenze University Press 
mondiale e l'avvento del fascismo; e soprattutto verificare a quali combinazioni i tre lemmi pervengano all'interno delle riflessioni rosselliane.

Occorrerà inoltre muoversi sulla linea di congiunzione tra teoria e prassi, tra speculazione e proposta politica. Non potrebbe essere diversamente, dato che questa ricerca ruota intorno alle idee di un uomo d'azione dotato di attitudine al contempo analitica e programmatica. In effetti, raramente come nel caso di Rosselli la storia del pensiero deve tenere conto della storia dei contesti. Rosselli rientra a pieno titolo nella categoria dell'intellettuale militante contemporaneo, che emerge nel clima di rottura di inizio novecento e all'accademia preferisce, o è costretto a preferire, la battaglia delle idee fusa (e a volte confusa) all'impegno diretto nell'agone politico.

Il confronto di Rosselli con le categorie del politico non è un confronto teorico in senso astraente. Rosselli non si muove mai su un piano intellettuale metapolitico. La maggior parte dei suoi scritti coincide con l'intersezione tra motivi teorici e motivi polemici.

Le considerazioni sul liberalismo non prescindono dal richiamo implicito alle teorie dei classici, ma sono gli atteggiamenti e le responsabilità dei liberali italiani a identificare il centro dell'attenzione.

La democrazia è sì pensata a partire dai princìpi fondamentali delle dottrine liberaldemocratiche, ma lo sguardo è rivolto essenzialmente al problema della crisi dello stato liberale italiano, che è crisi di impotenza rappresentativa nel quadro delle circostanze che hanno condotto all'affermazione del regime fascista.

Infine, le valutazioni sul socialismo attengono certo alla disputa su sistemi teorici e modelli sociali, ma riguardano in primo luogo il giudizio sui limiti della cultura politica espressa dal PSI e l'individuazione di possibili vie di rigenerazione ideale e rilancio politico.

Pur non prestandosi a ricomposizioni sistematiche, il pensiero di Rosselli può essere sintetizzato attraverso la raffigurazione di un duplice impegno critico. Fin dagli scritti più acerbi sull'interventismo, il suo percorso intellettuale matura e si consolida tramite una "partita" giocata su due fronti.

Il primo fronte coincide con la presa di distanza dal liberalismo illuminato assorbito nell'ambiente familiare e il progressivo avvicinamento alle ragioni del socialismo. Non si tratta di una frattura violenta e irrimediabile. Piuttosto, della crescente consapevolezza di un deficit di sensibilità e apertura democratica dell'intellighenzia liberale italiana, anche nelle sue espressioni più alte e apprezzabili. La querelle con Luigi Einaudi acquista sotto questo profilo valore emblematico. La distinzione tra liberalismo come metodo e liberalismo come sistema è il criterio-perno su cui ruota la denuncia dell'arroccamento ideologico di chi, non volendo discernere lo spirito etico-politico del liberalismo dal modello economico capitalistico, finisce col negare le richieste di una libertà più equa e favorire la conservazione sociale.

Il secondo fronte vede Rosselli armato contro la bastiglia dottrinaria del marxismo, nella versione tardo-positivista largamente diffusa negli ambienti socialisti. È una sfida ingaggiata per ragioni che poco hanno a 
che fare con lo zelo filologico delle controversie esegetiche. L'antimarxismo di Rosselli discende da insofferenza filosofica, volontà di innovazione culturale, impellenza politica: spinte tanto forti da indurre alla liquidazione di una Weltanschauung alla quale pure si riconosce radicata valenza "mitologica", se non epistemica. Dal convincimento che la sconfitta del movimento socialista sia derivata da un corto circuito di fatalismo e rigidità dogmatica, deriva l'insistenza sulla necessità di ripartire dalla combinazione di intransigenza morale e apertura ideologica.

Dall'incrocio dei due momenti critici prende corpo un coagulo di idee che imprime al socialismo una curvatura in senso radical-democratico.

È la democrazia l'idea-forza che dovrebbe fungere da catalizzatore centripeto per coniugare difesa delle libertà civili e giustizia sociale; riconoscimento dei diritti dell'individuo e accesso diffuso e dal basso a nuove forme di partecipazione politica, sociale ed economica; rispetto delle differenze e promozione di maggior eguaglianza.

Una concezione democratica prescrittiva, quella di Rosselli; il quale, attingendo ad ascendenze eterodosse, in particolare di matrice anglosassone, inietta nel socialismo anticorpi libertari che si traducono in diffidenza verso le formule del collettivismo statalista e preferenza per soluzioni che esaltino l'autonomia, la cooperazione, il pluralismo.

Rosselli fu innanzi tutto un antifascista, e lo fu a tutto tondo. L'opposizione al regime è la bussola che orienta le scelte di vita del giovane borghese maturato nell'immediato primo dopo-guerra, così come del leader di GL fuggito da Lipari. L'antifascismo guida in Rosselli l'azione del presente e ispira la visione del futuro. Impossibile dunque trascurare il fattore F. ${ }^{4}$.

Negli scritti di Rosselli ritroviamo la resistenza al restringimento degli spazi di partecipazione libera e attiva sacrificati all'organizzazione del consenso plebiscitario; il valore del pluralismo politico, sociale e culturale minacciato dal conformismo autoritario; la rivendicazione di una più efficace e radicata democrazia economica contro il corporativismo illiberale. Idee che compongono il discorso di quella che è stata per diversi anni, durante il fascismo, un'esigua minoranza.

Al conflitto tra fascismo e antifascismo è applicata la logica dell'opposizione binaria amico-nemico. Il fascismo è il nemico totale, col quale non è concepibile scendere a compromessi e che va abbattuto con una rivoluzione democratica che, negli auspici di Rosselli, instauri una repubblica socialista. Perché questo è l'obiettivo politico, e si farebbe torto a Rosselli se si edulcorassero le sue convinzioni per renderne più "seduttivo" il ricorso come ispiratore di una sinistra riformista post-muro di Berlino. E tuttavia, traspare continuamente nelle pagine rosselliane una fede democratica non negoziabile.

${ }^{4}$ Cfr. D. Cofrancesco, GL e il fattore F. Come non leggere Carlo Rosselli, in M. Degl'Innocenti (a cura di), Carlo Rosselli e il socialismo liberale, Lacaita, ManduriaRoma-Bari 1999, pp. 177-185. 
Rosselli non si spinge mai a prefigurare il rigetto delle regole procedurali della democrazia formale in nome delle ragioni superiori della democrazia sostanziale. Il principio maggioritario affermato attraverso libere elezioni rimane sacro, così come sacri sono i diritti delle minoranze. L'affermazione di un sistema sociale ed economico progressivo è un fine politico che non può raggiungersi sacrificando l'ordinamento giuridico e istituzionale della democrazia. Il socialismo liberale non coincide con una teoria statuale incentrata sul modello del doppio stadio. La rivoluzione antifascista, giustificata dalla presenza della dittatura, non mira a impiantare la libertà politica strumentalmente. La democrazia rappresentativa non è insomma il cavallo di Troia della dittatura del proletariato. Su questo punto le idee di Rosselli non appaiono equivoche. Rispetto alla conformazione dello statuto albertino, la forma di stato deve essere modificata in senso repubblicano, federalistico, sociale (criteri sui quali i soggetti politici dell'antifascismo dovrebbero convergere come si converge su contenuti minimi condivisi che cementano l'alleanza resistenziale). Tuttavia, nessun modello alternativo al binomio libertà civili-libertà politiche è considerabile tappa successiva e superiore della civiltà democratica. Lo stesso interesse per l'autogoverno di fabbrica e il cooperativismo attiene al campo di un'integrazione e non di una sostituzione dell'ordine democratico. Rosselli non avrebbe mai sottoscritto la seguente affermazione di Lenin: «ogni tentativo, diretto o indiretto, di considerare la questione dell'Assemblea costituente dal lato formale, giuridico, nel quadro della comune democrazia borghese senza tenere conto della lotta di classe e della guerra civile, significa tradire la causa del proletariato, passare alla causa della borghesia $»^{5}$; non avrebbe approvato il freddo realismo che individua negli istituti democratici «un semplice strumento del quale le diverse classi possono e devono servirsi (e sapersi servire) per i loro fini di classe ${ }^{6}$. Difficilmente, in sostanza, avrebbe assecondato l'idea che il verdetto di elezioni regolari sia suscettibile di aggressione eversiva nel caso in cui risulti sfavorevole alla propria parte politica.

Il socialismo liberale è per Rosselli intimamente democratico, con tutte le tensioni problematiche che possono derivare dal tentativo di condurre a sintesi tre termini potenzialmente non componibili.

${ }^{5}$ Lenin, Tesi sull'assemblea costituente, in Id., Opere scelte, IV, Editori Riuniti, Roma 1975, p. 527.

${ }^{6}$ Lenin, Le elezioni all'assemblea costituente e la dittatura del proletariato, Ivi, V, p. 536. 


\section{CAPITOLO PRIMO}

\section{LA FORMAZIONE GIOVANILE}

\section{L'ambiente familiare}

Le origini della sensibilità culturale di Carlo Rosselli sono immerse in un milieu di provenienza non trascurabile. L'intreccio tra la vicenda umana, intellettuale e politica di Carlo e l'universo di Casa Rosselli è stringente e ricco di sfaccettature ${ }^{1}$. Una famiglia di famiglie, si potrebbe definire quell'insieme di nuclei parentali legati da ragioni affettive e affine retroterra, che fungeva anche da rete di solidarietà per Amelia Rosselli e i suoi figli².

Le Memorie di Amelia e l'Epistolario familiare sono le fonti che restituiscono i valori, le idee, i pensieri, i dolori e le speranze, assimilati da Carlo Rosselli dall'infanzia agli anni della guerra.

Le pagine che raccolgono i ricordi della madre e il dialogo con i figli offrono il ritratto di quella borghesia illuminata di origine ebraica, colta e liberale, che Leo Valiani ha definito il «meglio della borghesia, amante delle lettere, di musica, teatro, arti figurative, conoscitrice della vera cultura, che non è nazionale soltanto, ma cosmopolita $»^{3}$. Ma questi scritti suggeriscono anche l'immagine di un ponte tra un'epoca e un'altra: un ponte scosso dalle tensioni sotterranee di inizio novecento e bombardato dall'irruzione della guerra.

${ }^{1}$ Casa Rosselli è il titolo del volume di Giuseppe Fiori che ricostruisce le vicende di Carlo, Nello, Amelia, Marion e Maria (Einaudi, Torino 1999). Le due biografie classiche su Carlo Rosselli sono quella di A. Garosci, Vita di Carlo Rosselli, 2 voll., Vallecchi, Firenze 1973 (ed. orig. 1945) e quella di N. Tranfaglia, Carlo Rosselli dall'interventismo a "Giustizia e Libertà», Laterza, Bari 1968.

${ }^{2}$ Si trattava di una «serie di famiglie ebree che rappresentavano il cuore delle relazioni familiari e sociali dei Rosselli: i Nathan, i Levi, i Sereni, gli Zabban, gli Olivetti, gli Orvieto ecc.» (Prologo a Z. Ciuffoletti (a cura di) I Rosselli-Epistolario familiare 1914-1937, Mondadori, Milano 1997, p. XXXVII).

${ }^{3}$ Introduzione all'edizione SugarCo (1979) dell'Epistolario Familiare, pag. 14. Anche Stanislao G. Pugliese ha sottolineato l'appartenenza delle famiglie Rosselli e Pincherle (Pincherle Moravia è il cognome di Amelia da nubile) a un'«aristocrazia culturale urbana, cosmopolita e poliglotta, con una solida posizione sociale e sicura delle proprie possibilità» (S.G. Pugliese, Carlo Rosselli. Socialista eretico ed esule antifascista 1899-1937, Bollati Boringhieri, Torino 2001, p. 13). 
Il memoriale di Amelia racchiude una testimonianza che offre più livelli di lettura. È possibile scorgervi il passaggio dall'infanzia felice nella luminosa Belle Époque della Venezia di fine ottocento ${ }^{4}$, alle ansie crescenti per le traversie familiari ${ }^{5}$, le sofferenze e i lutti della guerra, prima, e del fascismo, poi.

E una retrospettiva esistenziale, biografica e storica quella consegnata alle pagine di Memorie. Sullo sfondo di cambiamenti epocali si annodano i fili della perdita ${ }^{6}$, così come il richiamo ai punti cardinali di una integrità morale e civile trasmessa attraverso l'educazione e declinata con rispetto e autonomia dai figli.

Amelia si sofferma sulla relazione tra le origini ebraiche e il profondo sentimento patriottico: l'assimilazione come conquista ${ }^{7}$; l'italianità come segno nobiliare recentemente acquistato partecipando attivamente alla lotta contro la dominazione austriaca ${ }^{8}$; la religione come saldo e intransigente ancoraggio etico nella vita privata e pubblica ${ }^{9}$. Esemplare questo passo:

[...] anch'io perciò, cresciuta in quell'ambiente profondamente italiano e liberale, non serbavo, della mia religione, che la pura essenza di essa dentro il cuore. Elementi religiosi puramente di carattere morale: e fu questo l'unico insegnamento religioso - se così si può chiamare, e che piuttosto che insegnamento era ispirazione - da me dato ai miei figlioli. Ricordo che il primo anno in cui mi trasferii a Firenze coi bimbi ebbi subito occasione di fare affermazione di questa italianità che non ammetteva due patrie ${ }^{10}$.

${ }^{4}$ Lo stesso titolo del capitolo dedicato agli anni della fanciullezza e dell'adolescenza è evocativo del clima: Balconi sul Canal Grande, in A. Rosselli, Memorie, a cura di M. Calloni, Il Mulino, Bologna 2001, pp. 35-106.

${ }^{5}$ Dopo dieci anni di matrimonio trascorsi tra Vienna e Roma, Amelia si separa dal marito Joe. Si trasferisce nel 1903 a Firenze, in condizioni economiche precarie a causa dell'insipienza con la quale il marito aveva amministrato i beni di famiglia. Il motivo della separazione è però di «ordine morale»: un tradimento che «doveva lasciare una traccia indelebile di dolore» nella vita di Amelia (ivi, pp. 112-113).

${ }^{6} \mathrm{Il}$ primogenito Aldo muore nel marzo del 1916 sul fronte dell'Isonzo. Carlo e Nello verranno trucidati dai sicari della Cagoule il 9 giugno del 1937 (cfr. M. Franzinelli, 9 giugno 1937. Il delitto Rosselli. Anatomia di un omicidio politico, Mondadori, Milano 2008).

${ }^{7}$ C. Vivanti (a cura di), Storia d'Italia, Annali 11: Gli ebrei in Italia, 2 voll., Einaudi, Torino 1997 e, con riferimento specifico alle famiglie veneziane dei Pincherle, dei Capon e dei Levi da cui proveniva Amelia, D. Calabi, Gli ebrei veneziani dopo l'apertura delle porte del ghetto: le dinamiche insediative, in Id. (a cura di), Oltre la serenissima, Istituto Veneto di Scienze Lettere ed Arti, Venezia 2001.

${ }^{8}$ A. Rosselli, Memorie, cit., pp. 127-28.

${ }^{9}$ Cfr. M. Viroli, Come se Dio ci fosse. Religione e libertà nella storia d'Italia, Einaudi, Torino 2009, pp. 309 e ss.

${ }^{10}$ A. Rosselli, Memorie, cit., p. 128. 
La tradizione familiare del marito confluiva e rafforzava la propria: «i parenti di mio marito, Nathan e Rosselli, erano stati, fin dagli anni della loro dimora a Londra, legati da intima amicizia con Mazzini ${ }^{11}$. E naturalmente Amelia rievoca il crepuscolo pisano di Mazzini in casa di Pellegrino e Giannetta Rosselli, zii del marito.

Sintetizzando: coscienza orgogliosa dello status di élite liberale colta; rigore morale alimentato dalla combinazione di profetismo ebraico secolarizzato ${ }^{12}$ e religione mazziniana del dovere; spiccato patriottismo risorgimentale; vocazione cosmopolita. La crescita dei fratelli Rosselli avviene sotto influsso di questi impulsi, trasmessi con dedizione e severità ${ }^{13}$.

Come reagirono in Carlo gli elementi dell'educazione materna el'ascendente delle figure d'entourage? Dal memoriale di Amelia sappiamo del «temperamento esuberante e generoso» e «dell'inadattabilità sua a rassegnarsi a qualsiasi fatto non gli andasse a genio» ${ }^{14}$; della decisione materna di fargli interrompere il Ginnasio per passare alla Scuola Tecnica, considerata la più spiccata attitudine per le scienze esatte; di uno sviluppo intellettuale piuttosto lento e confuso, compensato da un notevole recupero negli anni dell'adolescenza, in cui le conversazioni con lo «zio Giù» accendevano la passione per i temi socio-economici ${ }^{15}$; della richiesta di soddisfare a dodici anni un'impellenza religiosa, che non ebbe però seguito ${ }^{16}$. Attraverso le lettere di Carlo contenute nell'epistolario, e quindi per mezzo di una lente in larga misura retrospettiva, siamo a conoscenza della stima e dell'affetto per le figure più care: Gabriele Pincherle, fratello maggiore della madre, gli Zabban, i Ferrero ${ }^{17}$. Alle quali si aggiungono, in un ruolo di influen-

${ }^{11}$ Ivi, p. 108.

${ }^{12}$ Cfr. A. Cavaglion, L'ebraismo in Carlo e Nello Rosselli, "QCR», n. 11, 1998, pp. 69-75.

${ }^{13}$ Interessanti a questo proposito un paio di passaggi di argomento pedagogico. Nel primo Amelia rievoca le discussioni con l'amica Laura Orvieto «sul modo migliore di educare i nostri ragazzi. Essa mi reputava un po' troppo severa ed esigente. Esigente lo ero infatti, e lo riconoscevo. Ma ritenevo, e ho sempre ritenuta necessaria in fatto di educazione, una disciplina» (A. Rosselli, Memorie, cit., p. 119). Nel secondo, interrogandosi sulla bontà di un metodo educativo «intransigente», conclude: «Forse fu un bene. In questo senso: che la vasta apertura mentale di Carlo e Nello... che si schiuse in loro nella prima giovinezza, aveva come trampolino di partenza una base unilaterale. Mi è sempre sembrato...che offrire alle menti immature dei bimbi tutti gli aspetti di una verità e quindi tutte le possibili soluzioni, crei in essi una pericolosa confusione» (ivi, p. 129).

${ }^{14}$ A. Rosselli, Memorie, cit., pp. 123-124.

${ }^{15}$ Ivi, p. 118. Lo zio Giù e la zia Gì, Giulio e Giorgina Zabban, amici molto cari di Amelia e figure di riferimento per Carlo e Nello.

${ }^{16}$ Carlo andò un paio di volte «al tempio; poi, non ci volle più andare, né mai più, dopo allora, mostrò il più piccolo segno di una qualsiasi tendenza o preoccupazione religiosa...fatto uomo, Carlo non fu però ateo. Considerò, credo, le varie religioni quali manifestazioni di fenomeni storici» (ivi, p. 131).

${ }^{17}$ Cfr. M. Calloni, L. Cedroni (a cura di), Politica e affetti familiari. Lettere dei Rosselli ai Ferrero, Feltrinelli, Milano 1997. 
za e confronto sul piano dell'orientamento politico, quelle di Alessandro Levi $^{18}$ (cugino di Amelia) e di Gaetano Salvemini ${ }^{19}$.

Difficile stabilire l'esito alchemico degli influssi formativi sulla personalità di Carlo Rosselli. Un esito sicuramente dialettico, nel senso di assimilazione nel superamento. Se ne ha testimonianza in una lettera del 25 agosto 1928 dal confino di Lipari. Dopo la guerra, gli studi economici e l'avvio di una possibile carriera accademica, la decisione di dedicarsi esclusivamente alla battaglia politica antifascista, l'esperienza della rivista «Il Quarto Stato» con Pietro Nenni, l'impresa dell'espatrio clandestino di Filippo Turati e il conseguente arresto, Carlo si lascia andare a un bilancio senza rimpianti:

[...] e io confesso che questa vita, nonostante tutto, così come mi si viene ormai dipanando sotto gli occhi della mente, l'amo. Non saprei sceglierne una diversa. E sento anche che alla fabbricazione di questo filo hai tanto cooperato tu, mammà, che non vorrai, non saprai disconoscere mai il frutto delle tue fatiche. Così, in sostanza, ci hai voluti, anche se poi non in tutto e dappertutto senti di seguirci ${ }^{20}$.

I Rosselli erano giunti a Firenze nel 1903. Nel ricordo di Amelia, perfettamente integrata nell'élite culturale dell'Atene d'Italia, gli anni fino al 1914 furono «un periodo veramente meraviglioso, con una rinascita che pareva riallacciarla ai tempi remoti del suo glorioso passato, tanto si era diffuso l'amore per la cultura e per l'arte»; la madre dei Rosselli sostiene che, sebbene «altre influenze - di un diverso carattere - sorsero intorno a loro» in seguito, «l'impronta di quegli anni lontani» sia stata «incancellabile» ${ }^{21}$. La persistenza dell'impronta etico-estetica, propria di un ceto borghese colto, aperto, europeo, non sbiadita dunque dall'influsso di altre esperienze, in scenari più duri e nuovi, sotto la pressione delle scelte politiche.

La Firenze rammentata da Amelia Rosselli è la città dei fermenti culturali di inizio secolo, in cui si annidano volontà di cambiamento, insofferenza verso il passato, inquietudine e ansia di rottura. Un centro di ricezione e incubazione di tensioni attiviste, intuizioniste, antipositiviste e pragmatiste che rappresentano un propellente "filosofico" potenzialmente

${ }^{18}$ Cfr. A. Levi, Ricordi dei fratelli Rosselli, Centro editoriale toscano, Firenze 2000 (con introduzione di Simon Levi Sullam, un ricordo di Piero Calamandrei e postfazione di Lea Campos).

${ }^{19}$ Cfr. G. Salvemini, Carlo e Nello Rosselli, «Il Ponte», Anno VII, N. 5, maggio 1951 e G. Salvemini, prefazione a N. Rosselli, Saggi sul Risorgimento e altri scritti, Einaudi, Torino 1946. Sull'influenza di Salvemini e Alessandro Levi nei confronti di Nello, cfr. G. Belardelli, Nello Rosselli, Rubbettino, Soveria Mannelli 2007, pp. 69-70.

${ }^{20}$ Epistolario familiare, cit., p. 412.

${ }^{21}$ A. Rosselli, Memorie, cit., p. 122. 
disponibile a irradiarsi in diverse direzioni politiche ${ }^{22}$. Personaggi come Enrico Corradini, Giovanni Papini, Giuseppe Prezzolini e riviste come «Il Regno» o «La Voce» esprimono, da posizioni differenti, la stessa esigenza di nuovo non estranea anche a "L'Unità» di Salvemini ${ }^{23}$. Amelia Rosselli, senza reticenze, rievoca amicizie e simpatie: «ero a quel tempo legata da viva amicizia con Scipio Sighele, l'illustre irredento; con Enrico Corradini - da non dimenticarsi che parlo del 1905 o giù di lì - il quale aveva fondato, e dirigeva, il suo primo settimanale di carattere nazionalista $»^{24}$. Tra le simpatie, rientrano il «battagliero settimanale "La Voce», edito da un gruppo di giovani scrittori d'avanguardia, fra i quali primeggiava il Papini e la Società Leonardo da Vinci» ${ }^{25}$. Colpisce che, nei ricordi di una donna scevra da qualunque "eccesso", una nota encomiastica sia riservata al teppista Papini. In realtà, tanto nelle tentazioni nazionalistiche, quanto nel favore critico concesso alle "avanguardie", sono rintracciabili l'insofferenza per «un'epoca un po' bolsa del liberalismo italiano», la preoccupazione per un paese dall'aria "politicamente un po' stagnante», e il desiderio di «rivalutazione dei valori nazionali ${ }^{26}$.

È qui possibile intravedere il risvolto di due idiosincrasie: giolittismo e neutralismo. Due termini da considerare nell'accezione ampia e polemica assunta negli attacchi che puntavano ad aggredire la "vecchia" Italia imbracciando armi che da prevalentemente culturali divennero, con la guerra e nel dopoguerra, sempre più politiche ${ }^{27}$. Il primo racchiude i mali del parlamentarismo, del sistema clientelare, del compromesso astuto, delle pratiche di corruzione politica e morale. Il secondo, il tradimento degli ideali del Risorgimento, la traslazione a livello internazionale dei vizi di una democrazia imbelle ${ }^{28}$.

Certamente, la critica serrata nei confronti dell'Italia giolittiana, così come la convinzione delle sacrosante ragioni interventiste, rientreranno nel bagaglio ideologico (e anche psicologico) di Carlo Rosselli. La stessa maturazione del socialismo liberale conterrà le ragioni della distanza tanto dal riformismo quanto dal massimalismo, considerati a diverso ti-

${ }^{22}$ Cfr. E. Garin, Cronache di filosofia italiana 1900-1960, Laterza, Bari 1997, e in particolare il cap. V, dal titolo Positivisti e no, pp. 113-170 (ed. orig. 1955). 102.

${ }^{23}$ Cfr. E. Garin, La cultura italiana tra 800 e 900, Laterza, Bari 1962, pp. 77-

${ }^{24}$ A. Rosselli, Memorie, cit., p. 121.

${ }^{25}$ Ivi, p. 122.

${ }^{26}$ Ivi, pp. 111 e 121.

${ }^{27}$ Cfr. E. Gentile, Il mito dello stato nuovo dall'antigiolittismo al fascismo, Laterza, Roma-Bari 1982.

${ }^{28}$ Il risentimento per Giolitti e i neutralisti non verrà mai abbandonato da Amelia Rosselli. In una lettera a Carlo del 14 ottobre 1919, commentando il discorso programma di Giolitti tenuto a Dronero il 12 ottobre alla vigilia delle elezioni politiche, parlerà di «quell'uomo nefasto», capofila degli «infamissimi neutralisti» (Epistolario familiare, cit., p. 85). 
tolo esperienze interne a un'epoca da superare. Ma da superare come? Il percorso che comincia con l'entusiasmo irredentista e approda alla lotta antifascista è tutt'altro che scontato. L'esperienza della guerra attacca differenze e distinzioni consolidate, mescola campi e appartenenze, sollecita contaminazioni ideologiche che tendono a far saltare i riferimenti dell'età liberale. Rosselli affronta da giovanissimo il crinale stretto tra Apocalisse della modernità e Seduzione totalitaria ${ }^{29}$. Il fatto che le corde ancora acerbe della sua sensibilità culturale e politica lo spingano lontano da esiti nazionalistici e anti-democratici, testimonia dell'azione di anticorpi non necessariamente destinati a prevalere.

\section{L'esperienza della guerra}

In casa Rosselli la guerra inizia nel segno di un desiderio: che l'Italia rompa la neutralità e persegua il coronamento dell'ideale risorgimenta$1 \mathrm{l}^{30}$. Il fervore patriottico della madre contagia i figli: «la speranza di una guerra di liberazione di Trento e Trieste esercitava il suo terribile fascino su grandi e piccoli $\aleph^{31}$.

La sera del 25 maggio 1915 i tre fratelli spalancano la finestra e issano la bandiera ${ }^{32}$. Poi però la guerra mostra il suo volto feroce. Il primogenito Aldo muore nel marzo del 1916 sul Pal Piccolo. Carlo e Nello sono richiamati al fronte rispettivamente il 13 giugno del 1917 e il 20 aprile del $1918^{33}$. Tra il lutto e l'arruolamento i due Rosselli non vengono assorbiti nell'esasperazione dell'etica della guerra, bensì maturano il senso di un'etica nella guerra. Il conflitto non è vissuto con impetuosa disposizione da Sturm und Drang (che connotava l'interventismo più veemente, e spesso più retorico ${ }^{34}$ ); assume piuttosto il significato di un appuntamento col sa-

${ }^{29} \mathrm{Nel}$ volume L'Apocalisse della modernità. La grande guerra per l'uomo nuovo, Mondadori, Milano 2008, Emilio Gentile indaga le radici profonde del duplice sentimento che, negli anni della guerra, attraversò la cultura europea, divisa tra ansia palingenetica e angoscia della catastrofe. In La seduzione totalitaria, Donzelli, Roma 2003, Angelo Ventrone ha rintracciato nella grande guerra le origini di una radicalizzazione della politica dalle potenzialità deflagranti, che avrebbe sottoposto il modello liberal-democratico a tensioni di carattere sovversivo, contenenti i germi di una deriva totalitaria.

${ }^{30}$ In una lettera indirizzata a Carlo del 7 agosto 1914, 4 giorni dopo la dichiarazione di neutralità da parte del governo italiano, Amelia scrive: «mi piacerebbe parlare con te di questa guerra, che occupa il pensiero di tutti...spero anch'io che succeda quello che speri tu! Per noi è l'unica speranza (ma in cartolina non voglio spiegarmi di più)» (Epistolario familiare, cit., pp. 5 e 6).

${ }^{31}$ A. Rosselli, Memorie, cit., p. 139.

${ }^{32}$ Ivi, p. 140.

${ }^{33}$ Cfr. G. Fiori, Casa Rosselli, cit., p. 25.

${ }^{34} \mathrm{Cfr}$. A. D’Orsi, I chierici alla guerra. La seduzione bellica sugli intellettuali da Adua a Baghdad, Bollati Boringhieri, Torino 2005. 
crificio per una giusta causa, che poco ha a che vedere con l'intonazione bellicista della marinettiana «sfida alle stelle»"

All'inizio del 1917 risalgono i primi tentativi giornalistici. In gennaio, Carlo scrive con Nello l'editoriale d'esordio del giornale studentesco «Noi giovani». Lo stile è acerbo; il tono indulge alla retorica. Tuttavia, nelle poche righe di questo testo sono rinvenibili due capisaldi già solidi, che col tempo sosterranno il maturare della consapevolezza e dei contenuti politici: l'etica del dovere e l'universalismo democratico. Il titolo dell'editoriale è Il nostro programma e vi si legge:

Oggi si è capito che nemmeno noi giovani abbiamo il diritto di divertirci e di perdere il tempo mentre tutti lavorano...Come potremo essere allegri indifferenti quando tante energie, tante giovani vite si sacrificano ai più alti ideali?... Noi giovani sentiamo la responsabilità che ci pesa sopra: sentiamo la missione che ci attende. E per questo dobbiamo prepararci subito con tutte le nostre forze. È necessario che noi abbiamo una volontà forte e sicura, e una vera concezione del Bene e del Male. Basterà proporsi un Ideale, alto e nobile, puro e generoso: e a quello dirigere i nostri sforzi. Sia un ideale di giustizia e di felicità per tutti gli uomini ${ }^{36}$.

Parole da contrapporre a ogni catastrofismo decadente, e lontane dalla passione per la presunta valenza rigeneratrice della violenza ${ }^{37}$. Il linguaggio adottato tradisce semmai la propensione ingenua alla semplificazione manichea nell'esprimere il rifiuto di valori gerarchici e l'aspettativa che il male necessario del conflitto si rovesci nel bene della convivenza tra popoli di eguale dignità. Con idealismo mazziniano e desiderio di rinnovamento irenico, i Rosselli esprimono idee riconducibili al lessico etico-politico dell'interventismo democratico ${ }^{38}$.

L'interventismo di Carlo Rosselli è l'interventismo di Salvemini, che dalle colonne de "L'Unità» sovrapponeva alle ragioni del conflitto politico le ragioni del conflitto morale. L'irredentismo e gli ideali del Risorgimento si dilatavano nelle pagine di Salvemini fino a coincidere

${ }^{35}$ E. Gentile, "La nostra sfida alle stelle". Futuristi in politica, Laterza, RomaBari 2009.

${ }^{36}$ C. Rosselli, N. Rosselli, Il nostro programma, «Noi giovani», 1, gennaio 1917.

${ }^{37}$ Lo spirito di Rosselli non è assimilabile alla disposizione a tuffarsi «nella barbarie per rinvigorirsi»: così scriveva Prezzolini nell'articolo Facciamo la guerra, «La Voce», 28 agosto 1914.

${ }^{38}$ Un'interpretazione simpatizzante dell'interventismo democratico in C. Casucci, Introduzione a Interpretazioni del fascismo, Il Mulino, Bologna 1982, che riprende quanto sostenuto dallo stesso Casucci nell'articolo Interventismo democratico e prima guerra mondiale, "Il Mulino. Rivista bimestrale di cultura e di politica», 11, novembre 1967, pp. 952-962. Rilievi critici sono invece rinvenibili in G. Procacci, Gli interventisti di sinistra, la rivoluzione di febbraio e la politica interna italiana nel 1917, «Italia contemporanea», 138, marzo 1980. 
con la difesa della civiltà democratica in contrapposizione alla cultura autoritaria impersonata dagli Imperi Centrali ${ }^{39}$. Un tòpos, quello della dicotomia tra democrazie occidentali e regimi imperiali, che rinviava alla polemica su Civilisation e Kultur, stigmatizzata come impropria da Benedetto Croce ${ }^{40}$.

La rappresentazione del teatro di guerra come luogo della contesa tra libertà e autoritarismo, civiltà dei diritti e cultura militaresca, sogno di eguaglianza tra le nazioni e imperialismo, attraversava il fronte minoritario e trasversale dell'interventismo democratico. La differenza sostanziale rispet to all'interventismo nazionalista ruotava intorno a questa idea ${ }^{41}$, la cui nobiltà poteva finire per abbagliare l'intelligenza delle contraddizioni latenti nel cercare la pace giusta invocando lo stesso strumento brandito dal nazionalismo più aggressivo ${ }^{42}$. Argomenti simili, inoltre, identificavano una sponda difficilmente delimitabile da criteri rigidi di geografia delle appartenenze politiche, accomunando il radical-democratico Salvemini all'ancora repubblicano $\mathrm{Nenni}^{43}$, ai socialisti Ugo Guido e Rodolfo Mondolfo ${ }^{44}$. Con questi ultimi Rosselli entrerà in contatto attraverso Alessandro Levi; al 1926 risale l'avventura della rivista «Il Quarto Stato» con Nenni.

${ }^{39}$ Fin dall'inizio del conflitto, Salvemini scriveva: «la vittoria della Germania sulla Francia sarebbe considerata come la prova della incapacità della democrazia a vivere libera accanto ai regimi politici autoritari, e scatenerebbe su tutta l'Europa i danni e le vergogne di una lunga reazione antidemocratica» (G. Salvemini, Fra la grande Serbia e una più grande Austria, «L'Unità», 1914, III, 32, 561-62, ora in F. Golzio, A. Guerra (a cura di), La cultura italiana del '900 attraverso le riviste, V, Einaudi, Torino 1962, p. 425).

${ }^{40}$ Nella sua difesa della patria culturale di Winckelmann, Croce indicava l'insensatezza di applicare al giudizio sulla guerra le «vacuità teoriche sugli ideali democratici e sul regno della pace e della giustizia», in B. Croce, Pagine sparse, Laterza, Bari 1928, p. 61 (ed. orig. 1919).

${ }^{41} \mathrm{Cfr}$. R. Vivarelli, Storia delle origini del fascismo. L'Italia dalla grande guerra alla marcia su Roma, I, Il Mulino, Bologna 1991, pp. 124 e ss.

${ }^{42} \mathrm{Nel} 1932$ Rosselli rivedrà in modo autocritico l'esperienza dell'interventismo: «la nuova generazione intellettuale, la nostra generazione, volle l'intervento dell'Italia in guerra o vi aderì fiduciosa; lo volle... nella convinzione profonda che si servisse in tal modo la causa della libertà e della pace. La generazione di Turati si oppose...si deve ben riconoscere che non noi eravamo nel giusto, non noi interpretavamo la volontà delle masse, ma piuttosto Turati» (C. Rosselli, Filippo Turati e il socialismo italiano, "Quaderni di Giustizia e Libertà», n. 3. Giugno 1932, ora in Id., Scritti politici, a cura di Z. Ciuffoletti e P. Bagnoli, Guida, Napoli 1988, p. 261).

${ }^{43}$ Cfr. P. Nenni, Vogliamo la guerra perché odiamo la guerra, «Lucifero», 6 settembre 1914.

${ }^{44}$ Entrambi i fratelli Mondolfo presero parte al dibattito relativo alla guerra che si svolse su «L'Unità» dell'amico Salvemini. Su questo punto mi permetto di rinviare a C. Calabrò, Il socialismo mite. Rodolfo Mondolfo tra marxismo e democrazia, Polistampa, Firenze 2007, pp. 133 e ss. 
Come si è visto, "tutta la buona volontà di fare» ${ }^{45}$ che sorregge Rosselli durante il conflitto trova origine nell'habitus al contempo liberale e mazziniano appreso in famiglia. Con lo svolgersi degli eventi, la generazione della guerra ${ }^{46}$ è trascinata nella tensione che acuisce tanto il senso del sacrificio quanto la rivendicazione di un diritto a cambiare la storia e la società. Da questo punto di vista, la vicenda bellica è un acceleratore di prove e di aspirazioni estreme ed estremistiche. Rosselli assorbe con ricettività lo spirito del tempo e comincia a seguire una traiettoria compresa tra due poli. L'ispirazione patriottica spinge verso il romanticismo dei valori nazionali; alla coscienza del dovere nei confronti della patria si aggiunge una riflessione più profonda sulla questione sociale. Se ne può avere una prova dall'incrocio di due articoli dedicati agli avvenimenti che determinano una svolta nell'andamento della guerra, con implicazioni non solo militari ma anche ideologiche: l'inizio della rivoluzione in Russia e l'intervento americano al fianco dell'Intesa.

Gli articoli vengono pubblicati su «Noi giovani» e si intitolano emblematicamente Libera Russia e Wilson.

Sulle notizie che arrivano dalla Russia, i toni sono entusiastici. Rosselli vede nella rivoluzione di febbraio l'avvio di un grande rivolgimento politico in grado di generare il riscatto di un intero popolo. Lattenzione è catturata soprattutto dalla suggestione, che assume potenza iconografica, della dimensione collettiva: «era tutta una massa che saliva lentamente, inesorabilmente. La marcia si poteva ritardare, ma non impedire ${ }^{47}$. L'eccitazione è controbilanciata dalla cautela sui possibili esiti del sommovimento, che rischia di travolgere gli equilibri di un paese chiave nello scacchiere mondiale. Con prudenza liberal-democratica, Rosselli scrive: «queste riforme sono belle, alte; nobili, meravigliose. Ma datele piano, a poco a poco» ${ }^{48}$. Convivono dunque una speranza e una preoccupazione. La prima richiama la scoperta della massa, proiettata dal fronte italiano (sebbene Carlo non arrivi in prima linea) alle terre d'oriente. La guerra svela agli occhi del giovanissimo ufficiale di buona famiglia l'esistenza concreta delle disuguaglianze sociali. Qualunque sia l'ordine che succederà al disordine, la subalternità e l'esclusione delle classi popolari dovrà essere messa in discussione. La seconda è legata all'auspicio che il cammino dell'eguaglianza sia il prodotto della prudenza.

Wilson è una sorta di apologia dell'eroe democratico in grado di dare il battesimo a una nuova fase storica. L'autodeterminazione e la pace dei popoli sono i criteri che dovrebbero trasformare la politica mondiale, fino ad allora fondatasi sul principio di potenza. La guerra "giusta" è il sacrip. 66.

${ }^{45}$ Lettera di Carlo alla madre del 6 gennaio 1918, in Epistolario familiare, cit.,

${ }^{46}$ Cfr. R. Wohl, La generazione del 1914, Jaca book, Milano 1984.

${ }^{47}$ C. Rosselli, Libera Russia, «Noi giovani», n. 4, aprile 1917.

${ }^{48}$ Ibid. 
ficio necessario per affrancare l'Europa dalle tare delle monarchie dinastiche e liberarla, sul fronte delle relazioni internazionali come su quello dei rapporti sociali interni, dallo spirito oppressivo di conservazione che ne ha generato la crisi. Agli occhi di Rosselli,

Wilson è più che uomo. È il lato vero, il lato grande, immenso della guerra. Egli ha chiesto ai popoli di riunirsi tutti per un ideale comune. E per questo ideale irraggiungibile coi mezzi pacifici, egli ha dato il suo popolo, ha giuocato la sua popolarità ${ }^{49}$.

I due articoli sono comprensibilmente immaturi, più impregnati di idealismo sentimentale che di analisi politica. D'altro canto, l'orientamento ideologico è ancora vago e generico. Ciononostante, essi hanno rilevanza non trascurabile sotto il profilo della storia delle idee: sono da annoverare tra le testimonianze di una fase in cui le ragioni dell'interventismo democratico brillano di un breve «splendore $\aleph^{50}$.

Il 1917 non fu solo L'anno della disperazione ${ }^{51}$, in cui emerse con evidenza l'«inutile strage» condannata da Benedetto XV; fu anche un anno di illusioni e di speranze alimentate da prospettive convulse. La caduta del regime zarista in Russia e il tenore del messaggio wilsoniano influirono anche in Italia sul dibattito interno, scombinando schemi interpretativi e animando ipotesi di svolta inattese. In particolare, a sinistra transitò fugacemente l'idea che la guerra potesse risolversi non con il collasso dell'età liberale, bensì con la modernizzazione democratica della società. Turati manifestò ammirazione per quanto accadeva in Russia ${ }^{52} \mathrm{e}$ Antonio Gramsci - in una lettura sottesa da coerente intransigenza rivoluzionaria - riconoscerà il fascino insidioso esercitato dal wilsonismo tra le fila so-

${ }^{49}$ C. Rosselli, Wilson, «Noi giovani», n. 5, maggio 1917. L'euforia wilsoniana era sicuramente anche il frutto del confronto con Alessandro Levi, uno degli esponenti del socialismo riformista più vicini, insieme ai Mondolfo, alle posizioni dell'interventismo democratico. Rievocando gli snodi cruciali della guerra, Levi sosterrà che «innumerevoli migliaia di combattenti riconoscevano che nessuno aveva saputo indicare meglio del Presidente i fini ideali per i quali essi di momento in momento arrischiavano la vita» (A. Levi, Il pensiero politico di Woodrow Wilson, «Nuova Antologia», 16 marzo- $1^{\circ}$ aprile 1924, ora in Id., Scritti minori storici e politici, CEDAM, Padova 1957, p. 102).

${ }^{50}$ Cfr. V. Foa, Questo novecento, Einaudi, Torino 1996, p. 47.

${ }^{51}$ Cfr. A. Rati, 1917. L'anno della disperazione. Caporetto, Sometti, Mantova 2007.

${ }^{52}$ Turati si dichiarava convinto che «il grandioso avvenimento della Rivoluzione russa, col suo programma antiannessionista, in antitesi diretta e dichiarata ad ogni imperialismo» rappresentasse un punto di svolta incoraggiante del conflitto (F. Turati, Per la pace e pel dopo-guerra. Le rivendicazioni immediate del Partito Socialista, «Avanti!», 15 maggio 1917, e «Critica Sociale», 1-15 settembre 1919, ora in Id., Socialismo e riformismo nella storia d'Italia - Scritti politici 1878-1932, Feltrinelli, Milano 1979, p. 322). 
cialiste: «moltissimi socialisti» - scriverà nell'ottobre del 1918 - «anche fra i compagni italiani, non sono riusciti a sottrarsi all'incanto» e non sono risultati «immuni dalle contaminazioni che il loro spirito socialista può avere con le concezioni borghesi di equità e di giustizia ${ }^{53}$.

Nel periodo tra la primavera del 1917 e la fine della guerra, rivoluzione e democrazia avrebbero potuto insomma assumere il senso di parole accostabili, e non entrare in conflitto antinomico. Se rivoluzione democratica è una formula sintetica efficace per definire il pensiero di Carlo Rosselli ${ }^{54}$, forse è verosimile rintracciarne il bozzolo proprio in quei primi interventi capaci di captare, sebbene allo stato emotivo, le chances di riforma profonda dell'Italia e dell'Europa a partire dai messaggi che giungevano dalla Russia e dagli Stati Uniti. Nelle aspirazioni di Rosselli, emancipazione delle classi umili ${ }^{55}$ e giustizia internazionale assumevano valenza simbolica paradigmatica anche se indefinita. Avrebbero dovuto fungere da alfa e omega per il dopo guerra.

Ma il dopo guerra fu fonte di amarezza. Il wilsonismo mostrò presto la sua fragilità; lo scenario europeo non si acquietò nel disegno di una pace giusta, che sancisse il principio di autodeterminazione di popoli liberi e uguali. Versailles divenne il simbolo del fallimento, ipoteca su ogni progetto di rifondazione democratica e solidale del continente. Nonostante l'esperienza della guerra, l'Europa non giunse alla consapevolezza di essere permeata, come scriverà Rodolfo Mondolfo, da «dipendenza reciproca» $\mathrm{e}$ «solidarietà di fatto» ${ }^{56}$. Il nazionalismo esasperato non scompariva: si rimanifestava piuttosto fin dai termini di una pace punitiva che dava vita «ai rancori e agli odi di una folla di nazioni risentite o esasperate ${ }^{57}$.

Il quadro internazionale si riverberava su quello interno. Depressione economica ed esasperazione morale concorsero in Italia a esacerbare il clima ideologico. Sembrava compromessa la credibilità della vecchia classe dirigente liberale, incapace di interpretare fenomeni di massa resi

${ }^{53}$ A. Gramsci, Wilson e i socialisti, «Il Grido del Popolo», 12 ottobre 1918, ora in Id., Scritti Giovanili (1914-1918), Einaudi, Torino 1958, p. 318.

${ }^{54}$ Cfr. P. Bagnoli, Rosselli, Gobetti e la rivoluzione democratica: uomini e idee tra liberalismo e socialismo, La Nuova Italia, Firenze 1996 e F. Vander, Che cos'è Socialismo liberale? Rosselli, Gramsci e la rivoluzione in occidente, Lacaita, Manduria-Bari-Roma 2002.

${ }_{55} \mathrm{Nel} 1924$, rievocando l'esperienza della guerra, Rosselli sottolineerà l'effetto “epifania" generato dall'incontro con i soldati di estrazione non borghese: «a contatto col popolo, molti conobbero e apprezzarono la massa. Ne compresero i dolori, le lacune, le mirabili virtù. Io stesso ricordo con commozione la scoperta che ne feci e il grande amore che mi prese per essa» (C. Rosselli, Inchiesta sui giovani (Guerra e fascismo), «Libertà!», 15 maggio 1924, ora in N. Tranfaglia, Carlo Rosselli dall'interventismo a "Giustizia e Libertà», cit., p. 23).

${ }^{56}$ R. Mondolfo, La crisi contemporanea, in Sulle orme di Marx, vol. I, Cappelli, Bologna 1923, p. 33.

${ }^{57}$ Ivi, p. 32. 
ingovernabili dal malcontento e dalla disgregazione sociale. Come notò Turati, a irrompere era «uno stato di insurrezione psichica che non sarà domato se non da conquiste reali, radicali e profonde» ${ }^{58}$.

Già prima di congedarsi, Rosselli si rammarica nel percepire che l'Italia è «completamente a terra e si marcia fra le rovine $»^{59}$. Deluso e segnato, crede però ancora nelle risorse del combattentismo. È fermamente convinto che chi ha sofferto in trincea abbia il diritto-dovere di ricostruire il paese e di determinare una nuova fase politica e sociale. Risale al maggio del '19 un articolo che compare sulla rivista «Vita», pubblicata a Firenze sotto l'influsso de «L'Unità» di Salvemini. Lo scritto, che si intitola Compito nuovo ${ }^{60}$, condensa l'approssimativa posizione politica di Rosselli. La guerra conserva dei connotati positivi, ma sembra essere svincolata dal riferimento al mito risorgimentale. Diviene piuttosto un'esperienza collettiva, che dovrebbe fungere da improbabile collante nazionale. Resta perciò la fiducia nel movimento dei reduci, soprattutto nei tanti giovani che sono tornati dal fronte. Per Rosselli essi sono l'energia nuova del paese, in grado di determinare un'opera di svecchiamento e di rigenerazione nazionale. Con eccesso euforico scrive: «Oggi l'esser giovani è una forza, una spinta, è ragione d'orgoglio, si sente il bisogno dei vent'anni scapigliati che rivoltino questo mondo vecchio tumefatto. Luce, luce, aria nuova, gioventù, gioventù» ${ }^{61}$. Sono le nuove generazioni, che hanno condiviso il sacrificio della guerra pur provenendo da ceti diversi, a doversi riunire in «un fascio formidabile, pur non rinunciando ai loro diversi ideali sociali. Riunirsi in un fascio non significa rinnegare il proprio partito [...] $\mathrm{ma}$ al di sopra di tutto e di tutti: l'Italia» ${ }^{62}$.

E un linguaggio per certi aspetti non dissimile da quello che avrebbe potuto adottare uno dei tanti giovani reduci che aderirono ai fasci di combattimento. In effetti, dal 1919 al 1922 la polarizzazione estrema del quadro politico indusse a opzioni radicali. La retorica fascista faceva leva sulla difesa dei valori nazionali contro il pericolo rosso; il movimento socialista estremizzava le parole d'ordine rivoluzionarie accese dal mito bolscevico. In questa tenaglia, l'ideologia che aveva animato l'interventismo democratico risultava schiacciata ${ }^{63}$. Per tutti coloro che, appartenenti alla generazione della guerra, non potevano e non volevano riconoscersi nei riferimenti

${ }^{58}$ F. Turati, Rifare l'Italia. Un programma di azione socialista, in Socialismo e riformismo nella storia d'Italia, cit., p. 364.

${ }^{59}$ Questa la percezione di Rosselli in una lettera alla madre da Asiago del 27 agosto 1919, in Epistolario familiare, cit., p. 99.

${ }^{60}$ C. Rosselli, Compito nuovo, «Vita», 20 maggio 1919, ora in Id., Opere, vol.1, Socialismo liberale, a cura di John Rosselli, Einaudi, Torino 1973, pp. 5-9.

${ }^{61}$ Ivi, p. 5.

${ }^{62}$ Ivi, p. 7.

${ }^{63}$ Cfr. R. Vivarelli, Storia delle origini del fascismo. L'Italia dalla grande guerra alla marcia su Roma, I, cit., p. 152. 
politici dell'Italia giolittiana perché agitati da ansia di futuro, la scelta di campo avvenne in un clima esacerbato e caotico. Spesso con equivoci e ripensamenti. Basti pensare al caso di Ernesto Rossi, collaboratore del «Popolo d'Italia» fino alla marcia su Roma, strappato al fascismo da Salvemini e che con i Rosselli formò a Firenze la "piccola confraternita di salveminiani» ${ }^{64}$.

Rosselli diffida del bolscevismo, ma non lo teme ${ }^{65}$. Sebbene con un certo scetticismo, vagheggia la costituzione di un fronte interclassista e transpartitico in grado di disinnescare le asperità del nazionalismo senza rinnegare gli ideali patriottici e di promuovere riforme sociali incisive senza scatenare la lotta di classe ${ }^{66}$.

In una lettera del 27 novembre 1919, informa gli amici Zabban sul suo voto alle elezioni politiche: «Io ho pure votato (per la prima volta) e ho appoggiato la lista democratico-repubblicana combattente» ${ }^{67}$.

Sono gli anni in cui né Francesco Saverio Nitti né Giovanni Giolitti riescono a ricucire la lacerazione della società italiana con le istituzioni liberali. Gli anni in cui i due partiti di massa - socialisti e popolari - che nelle elezioni del novembre 1919 avevano ottenuto un largo successo di voti, non trovano alcuna forma efficace di mediazione all'insegna di una necessaria quanto improbabile "solidarietà nazionale". Gli anni delle indecisioni di Turati, che non spinge i riformisti parlamentari ad entrare al governo (ammesso che la rottura con il partito indispensabile per compiere il passo sarebbe valsa davvero a fermare il fascismo) ${ }^{68}$.

Rosselli riprende gli studi. Il 4 luglio 1921 la laurea al «Cesare Alfieri» di Firenze; il 9 luglio 1923 quella in giurisprudenza a Siena. Tema comune

${ }^{64}$ Cfr. G. Fiori, Casa Rosselli, cit., p. 35 e, dello stesso Fiori, Una storia italiana. Vita di Ernesto Rossi, Einaudi, Torino 1997. Cfr. inoltre gli Atti del Convegno tenutosi a Roma il 28 aprile 1988, dal titolo Socialismo liberale: Carlo e Nello Rosselli, Ernesto Rossi, Gaetano Salvemini, «Il Ponte», XLV, N. 5, settembre-ottobre 1989, pp. 156-206.

${ }^{65}$ In Compito nuovo scrive: «vogliamo conoscere ed essere conosciuti. Poter dire a questi giovani del popolo: - Tu sei socialista; benissimo; rispetto la tua opinione come la mia - . E se quest'uno ti dice: - io sono contro la borghesia - . Ecco, allora discutiamo. E tutto questo non per lottare con il socialismo, non per una paura cretina del bolscevismo che non verrà. $E$ se verrà non ci spaventa; siamo abbastanza giovani e abbastanza forti per lavorare materialmente» (cit., p. 9).

${ }^{66}$ In virtù di questa aspirazione, incerta e transitoria, Mario Isnenghi ha ritenuto di poter inserire Rosselli nella schiera di quei giovani intellettuali che, tra le fila degli ufficiali, maturarono una tendenza mentale frutto del duplice processo di "scoperta del popolo e rinnovata egemonia sul popolo attraverso la guerra» (Il mito della grande guerra, Il Mulino, Bologna 2002, p. 318).

${ }^{67}$ E aggiungeva: «anche Sandro Levi è scontento della stragrande vittoria socialista. Alla vittoria pericolosa si aggiunge, come non bastasse, l'impresa militarista dannunziana. Potrebbe essere che i due mali, le due forze avverse, neutralizzandosi, finissero per dare un vantaggio all'Italia» (lettera citata da Tranfaglia in Carlo Rosselli dall'interventismo a "Giustizia e libertà», cit., p. 31).

${ }^{68}$ Cfr. M. Salvadori, La sinistra nella storia d'Italia, Laterza, Roma-Bari 2000. 
alle due ricerche: i sindacati operai. Si avvicina al socialismo riformista, senza maturare una decisione di appartenenza partitica. La parentela con Alessandro Levi certamente incide ${ }^{69}$, ma più come tramite di incontri che sotto il profilo del condizionamento politico. Salvemini dirà che «allora egli era sotto l'influenza di Alessandro Levi, Rodolfo Mondolfo, la "Critica sociale" e Turati. Ma le radici liberal-democratiche rimanevano intatte nel suo spirito $»^{70}$. Scontata la gelosia retrospettiva del maestro paterno, il giudizio di Salvemini non può essere trascurato e merita una riflessione.

I biografi concordano sul fatto che dal 1919 al 1922 Rosselli si dedica prevalentemente alle sue ricerche ${ }^{71}$, anche se Tranfaglia ha parlato di «svolta» socialista avvenuta nel corso del 1921 e incoraggiata da due fattori convergenti: il diffondersi della violenza fascista, particolarmente in Toscana; e il prevalere, nell'universo interventista, della componente fanaticamente nazionalista e antipopolare su quella democratica ${ }^{72}$. Una svolta a sinistra attraverso lo studio e l'indignazione. Se però l'antifascismo, in senso negativo, deriva da un riflesso psicologico immediato, le coordinate ideologiche costruttive sono un'acquisizione progressiva e l'accostamento al socialismo è mediato dall'esigenza di individuare non tanto un soggetto partitico quanto un orientamento politico-culturale, che contemperi valori laico-progressisti della tradizione liberal-democratica nazionale e vocazione sociale.

Nel contesto dato, il riformismo socialista di Turati, Levi, Mondolfo, è ciò che meno si allontana dalle idee che Rosselli sta maturando. Ma con una riserva, che ritarderà l'adesione formale al 1924 - dopo l'assassinio Matteotti e quando già da due anni i turatiani hanno costituito un nuovo partito, il PSU - e che peraltro non si estinguerà, alimentando la polemica "eretica" nei confronti della vecchia guardia. Riserva concernente l'inadeguatezza del bagaglio ideologico riformista, in cui il marxismo positivistico e l'ancoraggio ai postulati della II Internazionale erano degenerati, secondo Rosselli, in prudenza corporativa e remissività politica, dopo la stagione eroica della crisi di fine secolo $^{73}$. Il periodo dedicato all'analisi

${ }^{69}$ In una lettera del 18 gennaio 1921 si fa riferimento alla partecipazione, insieme a Levi, al XVII Congresso nazionale del PSI. Nella lettera alla madre Rosselli afferma: «Sembra stamani che la scissione avvenga veramente a sinistra. Stamani, forse...vedrò Turati!» (Epistolario familiare, cit., p. 113).

${ }^{70}$ Lettera di Salvemini a Garosci, riportata in A. Garosci, Vita di Carlo Rosselli, cit., p. 28.

${ }^{71}$ Cfr. A. Garosci, Vita di Carlo Rosselli, I, cit., p. 23; S. Pugliese, Carlo Rosselli, cit., p. 24; G. Fiori, Casa Rosselli, cit., p. 30.

${ }^{72}$ Cfr. N. Tranfaglia, Carlo Roselli dall'interventismo a "Giustizia e Libertà», cit., pp. 45 e 50.

${ }^{73}$ A conferma di un rapporto tutt'altro che univocamente critico con il socialismo riformista, basterà citare un brano dell'articolo dedicato alla figura di Turati nel 1932, quando Rosselli ha per diversi aspetti radicalizzato in senso rivoluzionario la sua posizione politica. Le considerazioni si riferiscono al passato, ma è evi- 
economica dei sindacati serve in sostanza a Rosselli per «capire» ${ }^{74}$. Successivamente, affronterà in modo diretto e con maggiore coscienza l'attrito tra adesione istintiva, "generazionale", alla filosofia volontarista del «non-conformismo antigiolittiano» ${ }^{75}$ e socialdemocrazia.

\section{Studi sul sindacalismo}

Dal 1921 al 1923 Rosselli scrive le sue due tesi di laurea. Il primo lavoro, svolto sotto la guida di Riccardo Della Volta ${ }^{76}$, ha un'impronta prevalentemente storico-politica. Il secondo, più tecnico-economica. La lettura di questi studi giovanili suggerisce una spiccata inclinazione verso l'approccio delle scienze sociali. L'indagine su natura, organizzazione e compiti dei sindacati operai è la via analitica che implica giudizi di valore sull'essenza e sui destini del socialismo. Inoltre, le tesi riflettono il dibattito coevo, acceso in tutta Europa, sulla crisi della rappresentanza liberale ${ }^{77}$. Rosselli entra nel merito del confronto sui possibili assetti economici e sociali derivanti dalla spinta al riconoscimento delle nuove forze produttive emerse dall'inizio del '900; i temi sono quelli che ruotano intorno alla centralità del lavoro, declinabile in direzioni diverse e spesso antagonistiche: rappresentanza professionale, corporativismo, democrazia industriale, consiliarismo.

In particolare la prima tesi, dal titolo Il Sindacalismo, risente del clima ancora non del tutto sopito delle lotte sindacali culminate con le occupa-

dente che sono animate da valutazioni implicite sul presente: «prima di tutti, intuì il problema che si poneva alla sua generazione e agli inizi fu quasi il solo a capirlo. Vide cioè come l'immaturità proletaria non fosse che il riflesso della immaturità del paese, ancor prigioniero di forme di vita politica arretrate...e in conseguenza si batté non già per la creazione assurda...di una Italia socialista per la quale mancavano tutti i presupposti; ma per la conquista di una Italia moderna, liberale, che liquidasse gli avanzi feudali...; trasformò insomma un angusto problema di classe in un problema nazionale, e con ciò riuscì a assicurare al movimento socialista lunghissime simpatie in tutti i ceti. E fu propria del Turati questa concezione del movimento socialista come fattore di unificazione spirituale e di educazione nazionale; patriottismo assolutamente originale, patriottismo proletario, solo capace di risolvere i problemi di fronte ai quali era mancata la borghesia; patriottismo che senza sforzo e senza soluzione di continuità traboccava così nell'internazionalismo» (C. Rosselli, Filippo Turati e il socialismo italiano, cit., p. 227).

${ }^{74}$ Cfr. P. Calamandrei, Uomini e città della resistenza, Laterza, Bari 1955, p. 56.

${ }^{75}$ Cfr. D. Cofrancesco, Sul gramsciazionismo e dintorni, Marco, Cosenza 2001, p. 74 .

${ }^{76}$ Sull'importanza dell'incontro con Della Volta, vedi le preziose analisi di Giovanna Angelini in L'altro socialismo: l'eredità democratico-risorgimentale da Bignami a Rosselli, FrancoAngeli, Milano 1999, in particolare pp. 103-104 e 123-124.

77 Cfr. C. Carini (a cura di), La Rappresentanza politica in Europa tra Ottocento e Novecento, Centro Editoriale Toscano, Firenze 1993. 
zioni dell'estate 1920 e attinge alla letteratura che aveva animato i dissidi tra correnti rivoluzionarie e riformiste. Proprio dalle considerazioni sulle differenti matrici dottrinarie del movimento operaio, è possibile desumere una conferma dell'ambivalenza che connota un pensiero che è in cerca, diviso tra bisogno di fede e senso della concretezza.

Apparentemente, Rosselli sembra schierarsi senza tentennamenti per il metodo riformista. I giudizi sul sindacalismo rivoluzionario sono severi. La dottrina soreliana è contraddetta su più punti fondamentali ${ }^{78}$. Accesa da «febbre distruttiva» ${ }^{79}$, essa ha della società borghese e della lotta di classe un "concetto semplicistico» ${ }^{80}$, volto a radicalizzare i termini del conflitto facendo leva sulla mistica della purezza proletaria contrapposta alla decadenza borghese ${ }^{81}$. Riprendendo spunti contenuti in un volume di Max Ascoli, Rosselli individua nel sorelismo i germi di un irrazionalismo antropologicamente $"$ pessimista ${ }^{82}$, che trova riscontro nel mito della violenza come antidoto permanente alle degenerazioni causate dall'edonismo materialistico della civiltà liberale. Su questo punto, è possibile ravvisare «uno dei non pochi punti di contatto della dottrina sindacalista rivoluzionaria col nazionalismo per cui molti sindacalisti rivoluzionari accettarono la recente guerra con entusiasmo ${ }^{83}$. Entusiasmo, un termine che - può sorprendere - non piace a Rosselli, probabilmente perché allude al lessico del vitalismo fascista e contraddice l'etica della volontà disciplinata dal senso del limite e del dovere ${ }^{84}$. Sul suo giudizio, certamente influisce il riflesso condizionato della cultura liberaldemocratica, che induce

${ }^{78}$ Il confronto critico con il sindacalismo rivoluzionario è ingaggiato prendendo in considerazione specificamente Sorel. Non mancano riferimenti ad Arturo Labriola, di cui si cita Socialismo e riformismo, Nerbini, Firenze 1905 e a Enrico Leone, di cui è richiamato Il Sindacalismo, Sandron, Palermo 1907. Tuttavia, si tratta di riferimenti non approfonditi. Cfr. pp. 62 e 63 del dattiloscritto della tesi di laurea Il Sindacalismo del 1921, custodito presso l'Istituto storico della resistenza in Toscana, Firenze, Archivio di «Giustizia e Libertà» - Appendice, Piccoli fondi privati, Carte Maria Rosselli, Busta 2, Fascicolo 2.

${ }^{79}$ Ivi, p. 62.

${ }^{80}$ Ivi, p. 65.

${ }^{81}$ Con una lettura à la Eduard Bernstein, Rosselli sottolinea l'esistenza e la funzione di una parte della borghesia intellettuale, nella convinzione che «sempre più va formandosi nei tecnici, chimici, ingegneri, professori, medici, una coscienza di classe distinta diretta a valorizzare la loro posizione nei confronti della lotta tra capitale e lavoro che troppo spesso fa astrazione dalla loro esistenza» (ibid.)

${ }^{82}$ Ivi, p. 72. Lo studio di Ascoli cui Rosselli fa riferimento si intitola Georges Sorel, Delessalle, Paris 1921. Su questo punto mi è stato molto utile lo studio di Alessandra Taiuti, Un antifascista dimenticato. Max Ascoli tra socialismo e liberalismo, Regione Toscana, Consiglio regionale, Firenze 2006.

${ }^{83}$ C. Rosselli, Il Sindacalismo, cit., p. 72.

${ }^{84}$ Significativamente scrive: «Sorel esalta poi la moralità della violenza, tanto più che egli crede che la morale non sia un insieme di precetti, ma uno stato di entusiasmo» (ivi, pp. 69-70). 
a una reazione contro il viscerale odio antiborghese, in quegli anni trait d'union fra opposti estremismi ${ }^{85}$.

In effetti, sembrerebbe che nell'ottica rosselliana non ci sia spazio per distinzioni all'interno dell'ideologia rivoluzionaria, complessivamente gravida di potenzialità autoritarie:

[...] questo è l'errore profondo della dottrina e della pratica sindacalista rivoluzionaria, come pure della dottrina socialista rivoluzionaria; si astrae cioè dalla massa, da quella stessa massa che si vuole riscattare e redimere, e si finisce in pratica fatalmente con la dittatura della minoranza ${ }^{86}$.

È solo un accenno a una questione cruciale: il paradosso che rovescia il soggetto storico collettivo miticamente investito della missione rivoluzionaria, in reggimento di manovra guidato dall'alto. Per Rosselli la massa non coincide schematicamente con la classe in senso marxiano, semmai col popolo, un popolo legato in prima istanza dalla semplice solidarietà dei bisogni comuni:

[...] occorre tenere presente che la grande massa dei lavoratori, vive, soffre, lavora, soprattutto per l'oggi, ben pochi sono gli idealisti che riescono a posporre praticamente nella dura realtà quotidiana i loro interessi immediati, ad un alto interesse generale ed ad un ideale futuro della conformazione della società umana ${ }^{87}$.

Attraverso le indagini contenute nella seconda tesi di laurea, Rosselli riconosce al lavoro graduale, lento e "prosaico" delle leghe il merito oggettivo di aver innalzato concretamente la condizione della classe lavoratrice: i fatti

[...] hanno dimostrato chiaramente che sotto la spinta delle Unioni i salari, non solo di particolari categorie, ma della intera classe lavoratrice, si sono elevati grandemente. Per l'Italia a convincersene basterà seguire le pubblicazioni dell'Ufficio del Lavoro dopo il 1900, cioè dopo che venne riconosciuta la libertà di sciopero e la neutralità governativa nelle lotte tra capitale e lavoro. Gli stessi socialisti marxisti più ortodossi ebbero a riconoscerlo clamorosamente rinnegando la ferrea legge dei salari ${ }^{88}$.

${ }^{85}$ Cfr. D. Settembrini, Storia dell'idea antiborghese in Italia 1860-1989: società del benessere, liberalismo, totalitarismo, Laterza, Roma-Bari 1991.

${ }^{86}$ C. Rosselli, Il Sindacalismo, cit., p. 274.

${ }^{87}$ Ivi, p. 273-274.

${ }^{88}$ C. Rosselli, Prime linee di una teoria economica dei sindacati operai, Betti, Siena 2005, p. 95. 
Quasi un'ammissione dei benefici effetti del compromesso giolittiano. Se ci si limitasse a queste annotazioni, il quadro non differirebbe molto da quello tratteggiato in opere "classiche" del pensiero sindacal-riformista. Antonio Graziadei e Ivanoe Bonomi avevano offerto giudizi analoghi in studi noti a Rosselli ${ }^{89}$. Ma c'è un'altra faccia del discorso.

I progressi innegabili a vantaggio del lavoro, attuati con la gradualità del metodo riformista, nascondono e contengono un deficit d'anima. L'inquietudine della volontà che agita Rosselli si scontra con i limiti dell'economicismo evolutivo imputati alla prassi delle riforme, intesa come guida burocratica di un processo ineluttabile. Il riformismo, politico e sindacale, adagiandosi sulla fiducia positivista nel corso delle cose, rischia di risolversi in un quietismo arido e rinunciatario (e di soccombere di fronte agli assalti delle ideologie emergenti). Antideterminismo, concezione morale del socialismo, idea della lotta come principio motore della storia, ostilità contro il collettivismo accentratore ${ }^{90}$ e centralità dell'uomo considerato nella veste del produttore, sono motivi disseminati in entrambe le tesi di laurea e sembrano far rientrare dalla finestra il Sorel espulso dalla porta ${ }^{91}$. Laddove per il marxismo «lo svolgimento del processo sociale sarebbe in rapporto con la maggiore o minore concentrazione capitalistica ${ }^{92}$, Rosselli sottolinea piuttosto l'urgenza di introdurre un «elemento volontaristico nel compimento del rivolgimento sociale» ${ }^{93}$, nella convinzione che «la parte debole del socialismo sia quella morale» ${ }^{94}$. Fino ad affermare con particolare enfasi che

${ }^{89}$ Di Antonio Graziadei Rosselli cita Socialismo e sindacalismo: conferenza tenuta nel ridotto del teatro d'Imola il 15 settembre 1908, prima edizione, Mongini, Roma 1909, seconda edizione, Avanti!, Milano 1921 e aggiunge: «scritto prima della guerra e prima della sua conversione» al comunismo (C. Rosselli, Il Sindacalismo, cit., p. 65). Di Ivanoe Bonomi, il libro di riferimento è Le vie nuove del socialismo, Sandron, Milano 1907. Cfr. P. Favilli, Riformismo e sindacalismo. Una teoria economica del movimento operaio: tra Turati e Graziadei, FrancoAngeli, Milano 1983.

${ }^{90}$ C. Rosselli, Il Sindacalismo, cit., p. 128-130.

${ }^{91}$ Per la complessità del rapporto con Sorel, cfr. P. Bagnoli, Carlo Rosselli tra pensiero politico e azione, Passigli, Firenze 1985. Bagnoli sostiene che da Sorel «Rosselli mutua la concezione del socialismo come questione morale non legata alla legge economica assolutizzata da Carlo Marx» (pp. 110-111). Dello stesso Bagnoli si veda anche Suggestioni soreliane in Piero Gobetti e Carlo Rosselli, in AA.VV., Georges Sorel nella crisi del liberalismo europeo, edizioni Affinità elettive, Ancona 2001 (presentazione di Paolo Pastori, introduzione di Giovanna Cavallari), dove è sottolineata l'influenza pratico-politica più che teorica di Sorel su Rosselli (p. 243). Cfr. inoltre G. B. Furiozzi, Carlo Rosselli e Sorel, "Il Pensiero Politico», Anno XXXII, n. 2, pp. 262-270 e Id., Il socialismo liberale. Dalle origini a Carlo Rosselli, Lacaita, Manduria-Bari-Roma 2003, pp. 86-88.

${ }^{92}$ C. Rosselli, Il Sindacalismo, cit., p. 106

${ }^{93}$ Ivi, p. 107.

${ }^{94}$ Ibid. 
[...] è inutile affannarsi a negare il carattere religioso del socialismo; esso è; la massa convinta lo rende tale. Inutile affermare che il carattere essenziale del socialismo è l'economico; quando esso penetra nel popolo, sia pure facilitato nella penetrazione da questo suo innegabile carattere economico, si trasforma, si allarga. Il vero socialista è un religioso; il socialismo è fede ${ }^{95}$.

Una fede indispensabile a confrontarsi con una battaglia, materiale ma anche esistenziale, che ha i caratteri della fatalità ineludibile ${ }^{96}$ e in cui le armi ideologico-simboliche contano quanto e forse più di quelle economiche.

Tuttavia, per quanto sia innegabile l'urgenza volontarista che impregna il socialismo di Rosselli, conferendogli una coloritura morale non lontana da alcuni tratti sorieliani, lo sbocco dottrinario è distante da Sorel. Lo è tanto nello spirito liberale, quanto nella valorizzazione del principio democratico come criterio formale e sostanziale di lotta regolata e di emancipazione. Assolutamente contrario all'idea che «il grande germe di decadenza della nostra epoca si chiama democrazia $»^{97}$, Rosselli respinge l'estremismo antidemocratico che punta il dito contro il parlamentarismo, il trasformismo, la corruzione politica, il prevalere della chiacchera leguleia contro gli interessi popolari. Se queste critiche così diffuse tanto nella sinistra quanto nella destra eversiva ${ }^{98}$ hanno un fondamento, la risposta non consiste nell'ammettere che la democrazia è il male, ma nel sostenere che «la democrazia non è stata applicata, o è stata male applicata ${ }^{99}$. Riassumendo, dal monitoraggio critico sul sindacalismo traspare l'esigenza di un riformismo iniettato di nuova energia ideale, che sappia coniugare difesa dei diritti e aspirazione utopica; privo di connotazioni angustamente classiste, ma in grado di dare dignità e protagonismo effettivo al lavoro; capace di riconoscere l'improponibilità del ritorno alle strategie di gradualismo "meccanico" del periodo giolittiano, e che però non ceda alle lusinghe dell' iconoclastica antidemocratica. In sostanza, riferimenti da "terza via" lib-lab, in un contesto che alle terze vie di tal genere offrirà sempre meno spazio. Ma vediamo meglio gli accenni propositivi che pure non mancano in questi primi scritti.

Rosselli non si limita infatti a un'analisi critica delle forme sindacali messe a confronto. Tenta di delineare in positivo il ruolo che l'istituzione sindacato può avere nel contesto di un nuovo sistema sociale ed economi-

${ }^{95}$ Ivi, p. 180.

${ }^{96}$ La lotta di classe è una «fatalità storica» (ivi, p. 65) e, d'altro canto, «vi saranno sempre coloro che per una ragione o per l'altra predomineranno» (ivi, p. 117). Il socialismo acquisisce eticità anche nella misura in cui riesce a far prevalere le ragioni del merito, del valore e dell'intelligenza, mentre il "predominio è attualmente nelle mani e del ricco e dell'intelligente, ma più del primo che del secondo» (ivi, p. 118)

${ }^{97}$ Ivi, p. 72.

${ }^{98}$ Cfr. Z. Sternhell, Nascita dell'ideologia fascista, Baldini \& Castoldi, Milano 2008.

${ }^{99}$ C. Rosselli, Il Sindacalismo, cit., p. 72. 
co. Sullo sfondo si staglia il ventaglio di prospettive che ha alimentato il dibattito sui destini dello stato post-liberale: si va dall'idea dell'inclusione dei corpi intermedi produttivi nella sfera di un ordinamento statale capace di sintetizzare e trascendere gli opposti interessi (che troverà applicazione autoritaria con il consolidamento del fascismo), all'idea dell'autonomia del lavoro attraverso forme più o meno pluralistiche di democrazia industriale (condannata a non incontrare concreta realizzazione). La posizione di Rosselli è ancora incerta. Vi sono però spunti interessanti, che ritroveremo sviluppati negli studi economici pubblicati in seguito. Coerentemente con la sua posizione riformista, Rosselli non rinnega il ruolo in primo luogo "difensivo" delle organizzazioni operaie, che coincide con la rivendicazione di diritti, tutele e garanzie in grado di migliorare le condizioni lavorative all'interno del sistema economico vigente. Ma a un tempo sostiene che i sindacati e i Consigli di fabbrica devono impegnarsi nella realizzazione di una economia socialista, nella consapevolezza peraltro che ciò non può aver luogo attraverso una rivoluzione. Per cui, se il fine ideale può essere quello di consentire ai lavoratori di dirigere la produzione, non solo viene escluso il ricorso alla violenza, ma il processo di transizione a eventuali forme di democrazia diretta del lavoro è oggetto di cautela ${ }^{100}$.

Rosselli non prende in esame l'esperienza dell'«Ordine Nuovo», che peraltro non conosce direttamente. Da una posizione che è e resterà lontana dall'operaismo, guarda piuttosto all'esperienza inglese. Apprezza la politicizzazione dei sindacati attraverso la costituzione del Labour Party. E condivide l'atteggiamento riformista dei Consigli di fabbrica, che, in Inghilterra, «hanno per oggetto esclusivamente questioni che non toccano la produzione $»^{101}$. Ma ciò non esclude il riferimento, ancorché problematico, alla tradizione sempre britannica del gildismo, che legava la rievocazione delle gilde medievali al paradigma della democrazia economica esercitata dal basso. Se rispetto al mezzo è esplicitamente favorevole alla formula riformista, rispetto al fine della socializzazione economica Rosselli esprime ancora una posizione vaga. C'è sicuramente la percezione dei rischi derivanti dalla soluzione collettivistico-accentratrice del socialismo di Stato. È una soluzione che può determinare come conseguenza «un aumento vertiginoso della burocrazia, di quella burocrazia di cui oggi vediamo i funesti effetti, e un accentramento ancora più grande dell'attuale che ci porterebbe fatalmente alla dittatura di pochi uomini sul resto del proletariato ${ }^{102}$. Proprio da ciò deriva l'attenzione verso l'ipotesi di au-

${ }^{100}$ Ciononostante, nella tesi del 1923, Rosselli non esita a indicare come obiettivo ideale la «subordinazione integrale dell'elemento oggettivo (capitale) all'elemento soggettivo (lavoro)» e scrive che il cuore del problema è «la sostituzione delle forze organizzate del lavoro nella gestione e nella direzione delle imprese» (C. Rosselli, Prime linee di una teoria economica dei sindacati operai, cit., p. 68).

${ }^{101}$ C. Rosselli, Il Sindacalismo, cit., p. 164.

${ }^{102}$ Ivi, p. 184. La tendenza antistatalista è confermata anche in una breve recensione pubblicata nel dicembre 1921 su «Critica sociale», il primo intervento di 
togoverno industriale del gildismo. Il fatto è che Rosselli probabilmente non conosce ancora G. D. H. Cole, il più noto e influente teorico del selfgovernment gildista, e non è in grado di misurare la distanza precisa che separa la visione di Cole da quella dei coniugi Sidney e Beatrice Webb di Industrial Democracy ${ }^{103}$, testo che invece ha avuto modo di consultare. La teoria gildista è ripresa indirettamente, attraverso l'analisi dell'interpretazione datane dall'austro-marxista Rudolf Hilferding ${ }^{104}$, a conferma di una certa approssimazione nel distinguere fra le diverse anime del socialismo inglese. Sarà solo dopo un contatto diretto con la cultura laburista, attraverso due viaggi estivi nel '23 e nel '24, che Rosselli arriverà a esprimere un'ipotesi più convincente, propendendo per la soluzione di Cole piuttosto che per quella sostanzialmente statalista dei Fabiani. È importante però, sin d'ora, la tendenza verso un modello di organizzazione che configura un assetto democraticamente federale della struttura economica e industriale, nella convinzione che «il nuovo ordinamento della produzione dovrà inevitabilmente basarsi sui nuclei locali dei produttori» ${ }^{105}$. È questo il senso della citazione di Hilferding:

[...] perché la socializzazione non diventi un'organizzazione fiscale e burocratica della produzione, aggiunge lo Hilferding, "i rami di produzione socializzati dovranno essere gestiti da organi di "autogoverno" dell'industria", da "parlamenti" nei quali abbiano posto e voto coloro che hanno parte diretta nell'industria (personale direttivo, impiegati, operai), i consumatori della relativa industria, e, insieme con essi, i rappresentanti degli interessi generali, da nominarsi dagli organi centrali dell'economia ${ }^{106}$.

Rosselli sulla rivista storica del riformismo. Qui si può leggere che ricondurre il sindacato «nell'orbita dello Stato, significherebbe soffocarne lo sviluppo» (Recensione a E. Artom, Lineamenti della crisi sociale, "Critica Sociale», 1-15 dicembre 1921, ora in C. Rosselli, Opere (vol. 1), cit., p. 22).

${ }^{103}$ Nella bibliografia, posta in appendice alla prima tesi di laurea, non vi è alcun riferimento ad opere di Cole. Dei Webb vengono invece richiamate, oltre a Industrial democracy, Storia delle unioni operaie, Esame della dottrina sindacalista e The british labour mouvement under war pressure (Il Sindacalismo, cit., p. 302).

${ }^{104}$ Rosselli trae le considerazioni di Hilferding da un intervento al primo Congresso dei consigli di fabbrica tedeschi, tenuto a Berlino nell'ottobre del 1919, e di cui «Critica sociale» riportava un resoconto nella rubrica Sguardi in giro del n. 24, 16-31 dicembre 1929, pp. 382-383.

${ }^{105}$ C. Rosselli, Il Sindacalismo, cit., p. 185.

${ }^{106}$ Segue la considerazione che questi «singoli "parlamenti industriali" dovranno alla loro volta essere collegati tra di loro in un "parlamento supremo dell'economia" cui spetterà di fissare le direttive generali. Però, anche in questa industria socializzata, non deve introdursi una uguaglianza meccanica, né deve cessare la concorrenza. Il socialismo non significa assoluto livellamento, ma solamente "uguaglianza delle posizioni iniziali"» (ivi, pp. 185-86). 
Si tratta di una formula macchinosa e "sincretica", ma che corrisponde alle esigenze di conciliare le diverse sfumature della sensibilità rosselliana. Socializzazione sì, ma "partecipata", federale, democratica. Che tenga conto di interessi molteplici, cercando di conciliarli. Che coinvolga e responsabilizzi nella gestione e nella direzione, evitando il livellamento egualitaristico. Perché il movimento sindacale e cooperativo è visto come una forza «liberatrice, positiva, creatrice» ${ }^{107}$, ma il passaggio al governo diretto da parte dei lavoratori può anche coincidere con un miraggio insidioso.

${ }^{107}$ Cfr. M. Degl'Innocenti, Carlo Rosselli e il movimento sindacale: dalla tesi di laurea a Socialismo liberale, in Giustizia e Libertà nella lotta antifascista e nella storia d'Italia, La Nuova Italia, Firenze 1978, p. 58. 


\title{
CAPITOLO SECONDO
}

\author{
I CONTI CON IL LIBERALISMO
}

\section{Liberalismo e liberismo}

Prima ancora di conseguire la seconda laurea, Rosselli dimostra notevole dinamicità politica e culturale. Con impazienza tenta di trovare uno sbocco sia alle sempre più sicure convinzioni antifasciste, sia al desiderio di proseguire la ricerca economica in ambito accademico. A Firenze partecipa attivamente con il fratello al Circolo di Cultura, inizialmente un'associazione apolitica in cui si discute di questioni sociali, economia, letteratura. Il Circolo riunisce esponenti della borghesia umanistica cittadina, Piero Jahier, Alfredo Niccoli, Enrico Finzi, Gino Frontali, Piero Calamandrei, Ernesto Rossi e, fulcro animatore, Gaetano Salvemini. Diventa presto una piccola cellula dell'antifascismo democratico, duramente avversata dai fascisti fiorentini ${ }^{1}$. Contestualmente, Rosselli si muove tra Torino e Milano. $\grave{E}$ in cerca di contatti: vuol far conoscere i suoi studi e allargare la rete di relazioni. Nel dicembre del '22 si trova a Torino, per incontrare Pasquale Jannaccone, Achille Loria e Luigi Einaudi. A Torino tornerà anche nel febbraio del '23, senza peraltro ritenere particolarmente fruttuosi i risultati di questi incontri ${ }^{2}$. Più stimolante invece l'accostamento all'ambiente di «Rivoluzione liberale», presupposto di una collaborazione che segnerà gli anni '23-'24, attraverso una serie di articoli rilevanti nell'elaborazione della critica antiliberista e nella definizione teorica di un "liberalismo socialista". Risale al dicembre del '22 il primo incontro tra Rosselli e Piero Gobetti $^{3}$, e la frequentazione con il gruppo di giovani di via XX Settembre

${ }^{1}$ Cfr. P. Calamandrei, Il manganello, la cultura e la giustizia, in Non Mollare 1925. Riproduzione fotografica dei numeri usciti con tre saggi storici di Gaetano Salvemini, Ernesto Rossi e Piero Calamandrei, La Nuova Italia, Firenze 1955, p. 71 e C. Francovich, Il Circolo di Cultura: l'ultima espressione di vita democratica a Firenze, «Quaderni del Circolo Rosselli», XI, 1, gennaio 1991, pp. 80-84.

${ }^{2}$ Cfr. le lettere del 10 e del 12 dicembre 1922 e quelle del 28 febbraio, dell' 1 e del 2 marzo 1923, in Epistolario familiare, cit., pp. 132-33, 133-34, 148-49, 149-50, 150-51.

${ }^{3}$ Nella lettera del 17 dicembre del 1922, indirizzata alla madre, Carlo afferma: «dico a Nello che ho conosciuto Gobetti» (Epistolario familiare, cit., p. 137). Cfr. Z. Ciuffoletti, I fratelli Rosselli e Piero Gobetti, in S. Visciola, G. Limone (a cura di), I Rosselli: eresia creativa, eredità originale, Guida, Napoli 2005, pp. 73-84. 
sarà intensa, contrassegnata da affinità per comune propensione di spirito critico e volontà di cambiamento, ma anche da idee e proposte differenti ${ }^{4}$. Rosselli comunque non rimane a Torino. Dopo il conseguimento della laurea, ottiene attraverso Attilio Cabiati, cui era stato presentato da Salvemini, un incarico alla Bocconi come assistente di Einaudi per l'anno accademico '23-'245. Attraverso queste frequentazioni si attiverà anche, dall'inizio del 1924, una collaborazione con la «Riforma sociale». A Milano inoltre, accanto all'attività universitaria, in virtù dei rapporti con Claudio Treves e Turati, avvia sulla «Critica sociale» e sulla «Libertà» la sua riflessione "provocatoria" sull'urgenza di autocritica cui sottoporre il socialismo italiano ${ }^{6}$.

Negli anni che vedono prima Mussolini andare al governo e poi il fascismo consolidarsi e - nonostante la crisi Matteotti - tradursi in regime, Rosselli comincia ad acquisire visibilità. È uno dei giovani intellettuali politicamente attivi nella generazione che avversa il fascismo dalla prima ora. Elabora maggiori strumenti di comprensione e inserisce la sua voce in canali di dibattito riconducibili a uno spettro che va dal liberalismo laico al riformismo socialista. Critico dell'immobilità delle opposizioni, reagisce alla sindrome dell'impotenza con la ricerca "anticipata" delle ragioni della sconfitta. Un atteggiamento che è l'altra faccia di quella sorta di paralizzante rassegnazione che troverà nell'Aventino lo sbocco più emblematico. Rosselli cerca di individuare i limiti insiti nelle correnti politico-culturali che, sebbene minoritarie, avrebbero potuto contrastare più efficacemente l'involuzione autoritaria.

Gli interventi pubblicati in questa fase sono riconducibili a motivazioni contingenti eterogenee. Tuttavia, riordinati in prospettiva, assumono l'aspetto di un disegno teorico composito. Non una trama sistematica, che non corrisponde né alle intenzioni né alle attitudini speculative di Ros-

\footnotetext{
${ }^{4}$ Interessante in questo senso quanto sostiene Garosci: «Mentre le opposizioni classiche erano più o meno impotenti, un po' dappertutto si formavano gruppi di giovani che discutevano, cercavano contatti, reagivano in modo ancora elementare e per tentativi alla tirannide. Verso quest'epoca (e il dicembre 1922-marzo 1923 vede Rosselli a Torino) «Rivoluzione liberale» cessa a poco a poco di essere una rivista di "interpretazione" politica per diventare centro di collegamento antifascista", per concludere: «forze, al di fuori dei partiti, si ritrovavano su basi diverse da quelle delle organizzazioni classiche, abbozzavano movimenti nuovi. Essi rompevano con la tradizione di associarsi a movimenti culturali od estetici, politici soltanto nei loro riflessi, e mettevano al centro il problema etico - politico» (A. Garosci, Vita di Carlo Rosselli, cit., pp. 26-27).

${ }^{5}$ Cfr. lettera del 21 luglio 1923 da Marina di Pisa, in Epistolario familiare, cit., pp. 155-56.

${ }^{6}$ Nella lettera alla madre da Milano del 17 dicembre 1922 riferisce di un incontro fruttuoso con Treves; sempre nella corrispondenza con la madre è presente il riferimento a un incontro con Turati (lettera dell'8 marzo 1923), Epistolario familiare, cit., pp. 136 e 154. Su Rosselli a Milano cfr. N. Del Corno, Carlo Rosselli a Milano, «Nuova Antologia», fasc. 2244, ottobre-dicembre 2008, pp. 279-288.
} 
selli. Piuttosto un discorso in progress, al contempo critico e propositivo. Un itinerario in cui i rilievi polemici rivolti al dogmatismo della "scuola" liberista, così come le considerazioni sui cortocircuiti ideologici del socialismo riformista, convergono nella prospettiva di una sintesi teorica che troverà compiutezza in Socialismo liberale.

Dalla risoluzione della "crisi di fine secolo" in avanti, liberismo e giolittismo avevano assunto il senso di due visioni contrapposte del rapporto tra stato ed economia, potere politico e interessi sociali, amministrazione e società civile. Se a Giolitti e a Turati non poteva non essere riconosciuto il merito "liberale" di aver salvato l'Italia dalla svolta autoritaria del $1898^{7}$, il sistema di governo degli anni '10 esponeva il fianco ai criteri di giudizio variamente ispirati alla dottrina della libertà economica. Le accuse contro il protezionismo "consociativo" favorevole all' intesa tra grandi gruppi industriali e apparati sindacali, contro l'invadenza parassitaria dello stato nella regolamentazione della vita economica, contro la compressione delle energie sociali - in particolare al sud - che avrebbero potuto contribuire a modernizzare la società, provenivano da un fronte eterogeneo. $\mathrm{Da}$ Einaudi a Salvemini, da Arturo Labriola a Gobetti e Camillo Berneri, il lessico liberista trova spazio in Italia nella cultura liberale come in quella socialista, sindacalista rivoluzionaria, anarchica ${ }^{8}$.

Nei primi anni del governo fascista, «La Rivoluzione Liberale» è una tribuna che illustra efficacemente la molteplicità delle declinazioni liberiste. Il modello di governo conciliativo dei rapporti economici, espressione del compromesso giolittiano, è rigettato tanto da posizioni ostili alla lotta di classe, quanto da posizioni teoricamente favorevoli ad una maggiore radicalità e autonomia delle forze lavoratrici. Là dove Riccardo Bauer accusa il movimento sindacale di aver impropriamente subordinato la vocazione rivendicativa alle ragioni dell'ideologia socialista ${ }^{9}$, Berneri punta l'indice contro lo spirito statalista del socialismo italiano che ha irretito la combattività della classe operaia ${ }^{10}$. A un Einaudi che si oppone fermamente a ipotesi corporative di rappresentanza sociale istituzionalizzata ${ }^{11}$,

${ }^{7}$ Perfino Gobetti, uno dei critici più duri del "connubio" riformista, ammetteva «un primo periodo eroico» della «Critica sociale», in cui la rivista di Turati e Treves rappresentò «le esigenze di una garanzia liberale ('98)», «La Rivoluzione Liberale», Anno II, N. 6, 8 marzo 1923, p. 17 (da qui in avanti tutte le citazioni dalla rivista gobettiana sono tratte dalla edizione anastatica, Einaudi, Torino 2001).

${ }^{8}$ Cfr. M.E.L. Guidi, L. Michelini (a cura di), Marginalismo e socialismo nell'Italia liberale 1870-1925, Feltrinelli, Milano 2001.

${ }^{9}$ R. Bauer, Unità sindacale e cooperativa, «La Rivoluzione Liberale», Anno II, N. 6, 8 marzo 1923, p. 20.

${ }^{10}$ C. Berneri, Il liberismo nell'Internazionale, «La Rivoluzione Liberale», Anno II, N. 11, 24 aprile 1923, p. 46. In questo intervento Berneri sostiene che gli anarchici sono «i liberali del socialismo».

${ }^{11}$ L. Einaudi, Parlamento e rappresentanza di interessi, «La Rivoluzione Liberale», Anno II, N. 28, 25 settembre 1923, p. 114. Einaudi sostiene di essere contrario 
corrisponde un Arturo Labriola che giunge ad approvare le misure liberiste del ministro De Stefani, che proprio nel 1923 dispiegavano l'impegno del governo a riequilibrare in favore degli interessi capitalistici i rapporti di forza parzialmente modificati con la legislazione del dopoguerra e già aggressivamente sconvolti dagli attacchi del '20-'21 a tutta la rete organizzativa del lavoro (leghe, sindacati, cooperative) ${ }^{12}$.

$\grave{E}$ in questo contesto che trovano collocazione nella rivista di Gobetti gli interventi di Rosselli, che prendono spunto da un approfondimento dell'analisi economica dell'esperienza sindacale e sfociano nel tentativo di confutare la necessità teorica del connubio tra liberalismo etico-politico e liberismo economico. La polemica antiliberista è il primo tassello nel procedimento di distinzione del liberalismo in "metodo" e "sistema".

Nel marzo del '23 appare su «La Rivoluzione liberale» l'articolo Per la storia della logica, Economia liberale e movimento operaio ${ }^{13}$. Rosselli si impegna a dimostrare l'inconsistenza del dogma della libera concorrenza sotto il profilo delle funzioni e delle finalità del sindacato, sottolineando l'utilità economica, prima che politica, della tendenza "monopolistica" e "unitaria" delle leghe operaie.

La ragion d'essere dell'istituzione sindacale è, infatti, la limitazione della concorrenza nella vendita della merce lavoro, scopo che viene perseguito primariamente attraverso l'unione dei lavoratori in leghe operaie, successivamente attraverso il tentativo di contenimento della frammentazione in più leghe confliggenti. Se gli epigoni dell'economia liberale classica sono giunti, normalmente, a riconoscere «l'utilità, soggettiva e sovente oggettiva, alcuni financo la necessità, delle organizzazioni operaie $»^{14}$, ne condannano però aspramente la pretesa a costituirsi in un monopolio di rappresentanza, limitativo della stessa libertà di organizzazione. Di qui la denuncia di una contraddizione che, in termini di teoria liberista, si riassumerebbe nella domanda: «e perché, di grazia, la concorrenza tra le organizzazioni e non tra gli individui? E quale grande differenza può correre tra le due

«a queste sedicenti modernità legislative», che sarebbero un «regresso spaventoso verso forme medievali di rappresentanza politica».

${ }^{12}$ Secondo Labriola, «il lato socialmente utile, anzi progressivo, del fascismo è in questo suo rappresentare il capitale produttivo (agricolo ed industriale) contro il capitale e il lavoro improduttivi sperperati nella burocrazia statale procreata dalle statizzazioni e dalle municipalizzazioni, nelle false cooperative» (A. Labriola, Riformismo e cooperative, "La Rivoluzione Liberale», Anno II, N. 19, 19 giugno 1923, p. 79). Gli interventi del governo fascista dal 1923 al 1925 incisero in campo fiscale, con l'abbandono dei tentativi di perequazione: fu ad esempio accantonato il progetto di nominatività dei titoli azionari e ridotta l'imposta di successione. Cfr. G. Candeloro, Storia dell'Italia moderna. Il fascismo e le sue guerre, Feltrinelli, Milano 1993, pp. 48-49.

${ }^{13}$ C. Rosselli, Per la storia della logica, Economia liberale e movimento operaio, «La Rivoluzione Liberale», 15 marzo 1923, ora in Id., Opere (vol.1), cit., pp. 30-37.

${ }^{14}$ Ivi, p. 30. 
forme di concorrenza? $\aleph^{15}$. In realtà, per Rosselli, l'incoerenza rimanda a motivazioni di ordine ideologico piuttosto che economico:

[...] la libera concorrenza è la colonna centrale del sistema liberale; dogma fondamentale della scuola è il "libero giuoco delle forze economiche". Donde la condanna preventiva di quei movimenti e di quelle forze, in qualunque senso dirette, che attentino al sopradetto principio. Se però quei movimenti e quelle forze non accennano a scomparire, se anzi tendono ad affermarsi nella vita sociale, allora o vengono recisamente condannate ( $1^{\circ}$ tempo) o si tenta di costringerle, sia pure solo in teoria, nei limiti del sistema ( $2^{\circ}$ tempo). Così è accaduto pel movimento sindacale ${ }^{16}$.

Dove è facile intravedere l'ironico riferimento implicito a una costrizione che non si è verificata "solo in teoria".

Sarebbe quindi la costituzione teorica del modello liberale classico a scontrarsi col dato empirico, obbligando per reazione a irretire la forza espansiva del movimento sindacale, in quanto contrastante con le regole del mercato liberale. Rosselli ritiene che la tendenza monopolistica sia iscritta nel "codice genetico" del sindacato:

[...] in conclusione io affermo che dal lato economico, una volta ammesso che le leghe operaie sorsero e sorgono coll'intento precipuo di eliminare la concorrenza, si deve logicamente affermare che è naturale, giusto, necessario ch'esse tendano al monopolio. Nulla da obbiettare allorché si lotta contro questa o quella politica sindacale; ma da respingersi senz'altro l'astratta e dogmatica illazione antimonopolistica ${ }^{17}$.

Ma se il movimento sindacale rivela una esplicita vocazione antiliberista, come può rappresentare uno strumento efficace di reale azione liberale? La risposta di Rosselli rinvia a una contraddizione che non è del sindacato, ma del modello sociale della scuola liberista, la quale, pur richiamandosi a una visione antagonistica della società e condannando per questo gli "accordi parassitari" tra capitale e lavoro, vede nel conflitto tra queste due variabili una minaccia incombente per l'interesse generale. Così gli economisti liberali, che accanitamente denunciano «i colposi fenomeni di parassitismo di ceti capitalistici ed operai sfruttatori della collettività attraverso i dazi doganali, i premi di produzione, concessioni graziose, ecc. ecc.», non si chiedono se

[...] cotesti accordi colposi [...] non si moltiplicheranno grandemente allorquando, in un giorno ch'essi hanno sempre auspicato, ad una teo-

\footnotetext{
${ }^{15}$ Ivi, pp. 31-32.

${ }^{16}$ Ivi, p. 30.

${ }^{17}$ Ivi, p. 32.
} 
ria e soprattutto a una pratica del movimento operaio orientata in base al postulato della lotta di classe, si vada sostituendo una teoria ed una pratica collaborazionista (vedi organizzazioni bianche e tricolori) ${ }^{18}$.

In sostanza, l'auspicata frantumazione del fronte sindacale in nome della "libertà di associazione" non è destinata a determinare un regime di concorrenza funzionale al progresso economico e sociale, bensì l'addomesticamento del lavoro.

Dal punto di vista di Rosselli, la lotta di classe esiste quale dato empirico, in coerenza con una definizione del concetto di classe non marxista, ovvero secondo una visione dinamica della realtà sociale che esclude l'idea dell'orizzonte rivoluzionario palingenetico, ma non teme la modificazione progressiva degli equilibri sociali. È questa una ragione, almeno preliminare, d'intesa con Gobetti, il quale tuttavia associa il concetto di lotta di classe alla teoria dell'azione liberal-liberista e antiriformistica delle "aristocrazie operaie"19. In effetti, Gobetti, in contrasto con Croce, che aveva criticato la "assurdità logica" del concetto di lotta di classe, afferma che tale critica sarà valida

[...] contro la filosofia della storia di Marx e contro l'illusione messiani$\mathrm{ca}$, di natura mistica e hegeliana, di un'abolizione finale delle classi. In realtà la praxis ci addita ogni giorno in seno all'unità sociale il formarsi di classi distinte che, per legge naturale, si ipostatizzano, si associano, combattono per interessi presenti e per idealità future ${ }^{20}$.

Il punto importante della polemica antiliberista è che la classe sindacalizzata, nell'accezione dinamica e flessibile rosselliana, può avere una funzione essenziale non solo nell'ottica della trasformazione socialista, ma anche nell'ambito del sistema capitalistico. In Per la storia della logi$c a$, Rosselli abbozza un esame del movimento sindacale anche in chiave

${ }^{18}$ Ivi, pp. 32-33. La netta contrarietà a ipotesi "collaborazioniste" sembra non offrire conferma all'interpretazione di chi ha intravisto nella terza via rosselliana affinità col corporativismo fascista. Cfr. R. Chiarini, Il corporativismo paradigma del totalitarismo, "Nuova Storia Contemporanea», Anno IV, N. 1, gennaio-febbraio 2000, pp. 33-42.

${ }^{19}$ Cfr. ad esempio P. Gobetti, Liberismo e operai, «La Rivoluzione Liberale», Anno II, N. 8, 3 aprile 1923, p. 34, in cui si afferma che «un movimento operaio intransigente contro tutti i riformismi potrebbe segnare l'inizio della revisione e offrire i quadri per la lotta inesorabile del liberismo» e Id., Socialismo di Stato, «La Rivoluzione Liberale», Anno II, n. 15, 22 maggio 1923, p. 63. Cfr. P. Bagnoli, L'elitismo democratico in Italia: Gobetti, Dorso, Burzio, Rosselli, in E.A. Albertoni, P. Bagnoli, Studi sull'elitismo, Giuffrè, Milano 2001 ed E. Ripepe, Gli elitisti italiani, 2, Gobetti-Burzio-Dorso, Pacini, Pisa 1974.

${ }^{20}$ P. Gobetti, I torti della teoria liberale, in Id., La Rivoluzione liberale, Saggio sulla lotta politica in Italia, con un saggio introduttivo di Paolo Flores d'Arcais, Einaudi, Torino 1995, p. 47 (ed. orig. 1924). 
propositiva, associando alla suaccennata tendenza monopolistica la formazione di una organizzazione dei produttori che, attraverso una sorta di identificazione con la "comunità dei consumatori", garantirebbe la reale difesa dell'interesse generale. Questa tesi, che verrà approfondita e messa al servizio di una teoria di tipo cooperativistico-gildista, serve per adesso da sostegno al tentativo di confutazione delle posizioni liberiste. Rosselli giunge, infatti, ad affermare:

[...] sembra invece che la "lotta di classe", la lotta fra detentori di capitali e detentori di forza-lavoro eserciti una funzione utile e necessaria nella economia capitalistica. Se effettivamente non esistesse la classe bisognerebbe costruirla artificialmente. Giacché di fronte alla innegabile impotenza dei consumatori nel fenomeno dello scambio, di fronte al progressivo rafforzarsi della posizione strategica dei venditori attraverso monopoli e sindacati, l'ultima trincea che resta è quella del corpo organizzato dei produttori. Quanto più vasta ed unitaria sarà l'organizzazione dei produttori, tanto più il suo interesse si identificherà con quello della massa consumatrice ${ }^{21}$.

Da questa argomentazione emergono e sono da sottolineare due elementi. In primo luogo, il tentativo di sorreggere le motivazioni sociali della difesa del lavoro con un impegno demistificatorio in termini di analisi dei rapporti economici: i monopoli sono un dato della prassi dal lato della impresa, tale da smentire ogni modello teorico di libera concorrenza. In secondo luogo, la convinzione secondo cuil'interesse di una intera comunità non si persegue esclusivamente né con il libero mercato, né con un semplice processo rivendicativo-redistributivo sul fronte del consumo, ma con l'azione diretta e coalizzata dei lavoratori su quello della produzione.

\section{Il confronto con Einaudi}

Per la storia della logica segna lo spunto iniziale di una polemica, leale ma dura, tra Rosselli ed Einaudi. Sarà Einaudi, infatti, a rispondere severamente, sempre dalle colonne della «Rivoluzione liberale», all'articolo di Rosselli, denunciando la mancanza di qualsiasi riferimento bibliografico a supporto delle accuse contro la scuola economica liberale ${ }^{22}$. Rosselli re-

${ }^{21}$ E aggiunge: «per un gioco che appare dialettico si può affermare che la migliore difesa dei consumatori può essere raggiunta solo attraverso la loro coalizione in veste di produttori, vale a dire nel lato positivo dell'attività umana. I fenomeni di parassitismo e di accordi colposi col padronato sono tanto più difficili quanto più misera, debole, divisa, è la organizzazione generale dei produttori» (C. Rosselli, Per la storia della logica, cit., p. 34).

${ }^{22}$ In effetti Einaudi si limita a una reazione irritata di carattere "professorale". Scrive: «non entro nel merito dei problemi trattati...ma è evidente che in certi casi la 
plica con un nuovo articolo, apparso anch'esso sulla rivista di Gobetti, in cui fa riferimento alle opere di Giuseppe Prato e a interventi dello stesso Einaudi, per sottolineare quelle che gli sembrano Contraddizioni liberi$s t e^{23}$. Rosselli ribadisce la naturale vocazione monopolistica dei sindacati, monopolio che viene definito come obiettivo tendenziale, concretamente irrealizzabile, ma non per questo tale da pregiudicare, come invece pensava Prato, la spinta unitaria del moto operaio ${ }^{24}$. Con riguardo poi specifico ad Einaudi, egli riprende il tema della lotta di classe e, citando due articoli dello studioso torinese, individua una contraddizione nella pretesa di negarla quale "parto della "dottrinella marxista" , in nome di un astratto interesse nazionale, e nel contempo di deprecare i casi di collaborazionismo parassitario e di scendere in campo «contro il collaborazionismo fascista pei fatti di Livorno (concordato tra fascisti e cantiere Orlando)» ${ }^{25}$.

La critica a Einaudi continua e ha il suo momento culminante nella pubblicazione dell'articolo Luigi Einaudi e il movimento operaio ${ }^{26}$. Rosselli prende spunto dalla raccolta di scritti einaudiana Le lotte del lavoro ${ }^{27}$, edita da Gobetti, per sostenere un'interpretazione del liberalismo basato sulla distinzione tra metodo (liberale) e sistema (capitalistico-liberista), e per individuare nel movimento operaio la vera forza liberale del contesto storico contemporaneo. Nell'articolo viene criticata la posizione ideologica dell'economista piemontese, il quale negherebbe all'azione della classe operaia ogni valore di libertà, in quanto orientata in una prospettiva marxista e quindi, ipso facto, antiliberale. Rosselli attribuisce a Einaudi una visione dogmatica dell'integrazione "sistemica" tra liberalismo e capitalismo, dell'inscindibilità tra politica e struttura economica. Assumendo come premessa essenziale «la bontà - non assoluta ma relativa - la superiorità del regime capitalistico su ogni altro regime o sistema economico», egli rivelerebbe «il suo grande scetticismo nelle virtù costruttive del moto operaio, in quanto tende appunto a modificare e a rovesciare le basi economiche e morali della società attuale» ${ }^{28}$.

In realtà, Rosselli riconosce, e allo stesso tempo rimpiange, l'epoca in cui Einaudi guardava con favore alle lotte operaie. Ed è, in effetti, lo stesso Einaudi a rievocare «gli sforzi che nel 1897 e nel 1900 compievano alcuni

forma ed il metodo decidono il merito della controversia» (L. Einaudi, Esegesi delle fonti, «La Rivoluzione Liberale», Anno II, N. 9, 10 aprile 1923, p. 39).

${ }^{23}$ C. Rosselli, Contraddizioni liberiste, «La Rivoluzione Liberale», 24 aprile 1923, ora in Id., Opere (vol. 1), cit., pp. 38-43.

${ }^{24}$ Cfr. Ivi, pp.38-39.

${ }^{25}$ Ivi, p. 40. Gli articoli di Einaudi cui si fa riferimento furono pubblicati sul «Corriere della sera» l'11 e il 18 settembre 1922.

${ }^{26}$ C. Rosselli, Luigi Einaudi e il movimento operaio, «Critica Sociale», 15-31 maggio 1924, ora in Id., Opere (vol. 1), cit., pp. 44-51.

${ }^{27}$ L. Einaudi, Le lotte del lavoro, con una introduzione di Paolo Spriano, Einaudi, Torino 1972 (ed. orig. 1924)

${ }^{28}$ C. Rosselli, Luigi Einaudi e il movimento operaio, cit., p. 45. 
gruppi di operai italiani», definendo quel periodo «gli anni eroici del movimento operaio italiano ${ }^{29}$. Ma, come ha notato Paolo Spriano, «la fine secolo, il “dopo Crispi", il '98, l'inizio timido della svolta dopo la reazione, sono un momento che vede semplificati i termini di una scelta: di qua i progressisti, di là i reazionari $»^{30}$. La solidarietà progressista del liberale era allora solidarietà umana, rivolta quindi allo sforzo per l'emancipazione dal bisogno, per il miglioramento delle condizioni materiali, per la rivendicazione dei diritti civili e politici. Questa simpatia si tramuta però in drastica avversione di fronte al progetto rivoluzionario socialista, che stravolgerebbe il sistema economico e il regime delle libertà (intimamente collegati). Nel quadro dei rapporti d'impresa, il limite posto all'azione operaia è definito in modo inequivocabile. La dialettica capitalisti-lavoratori segue per Einaudi la logica della distinzione di funzioni:

Solo discutendo faccia a faccia, queste due razze di uomini possono giungere a riconoscere le proprie sovranità rispettive: l'uno sulla direzione, sulla organizzazione e sull'invenzione dell'impresa, l'altro sulla propria forza di lavoro [...] E' bene che ognuno custodisca gelosamente l'esclusivo dominio sul proprio compito che è, per l'imprenditore, di organizzare l'impresa e per l'operaio di prestare la propria opera manuale od intellettuale ${ }^{31}$.

Sul piano della libertà, poi, la condanna del socialismo è senza appello. Per Einaudi non c'è movimento socialista che non sia marxista, e in quanto tale inevitabilmente collettivista, classista, quindi antiliberale, anzi liberticida. Tale la repulsione da essere espressa in un passo dai toni esasperati:

Chi vide, raccapricciando, nel 1919 e nel 1920, le folle briache di saccheggio e di sangue per le vie delle grandi città italiane, non riconobbe i figli di quegli uomini, che dal 1890 al 1900 nascevano alla vita collettiva, comprendevano la propria dignità di uomini ed erano convinti dell'alta meta umana a cui aspiravano. Lo spirito satanico della dominazione inoculato da politicanti tratti dalla feccia borghese li travolse e li trasse a rovina ${ }^{32}$.

${ }^{29}$ L. Einaudi, Le lotte de lavoro, cit., p.11. Cfr. G. Pagano, Luigi Einaudi e il socialismo, Bibliopolis, Napoli 1993.

${ }^{30}$ E continua: «E nessuno è più liberale in quel momento, nel paese e nel parlamento, della pattuglia dell'estrema sinistra, del giovanissimo partito socialista che regge benissimo alla bufera dello scioglimento e delle repressioni» (Introduzione a L. Einaudi, Le lotte del lavoro, cit., p. XII).

${ }^{31}$ Ivi, p.10.

${ }^{32}$ Ivi, p. 11, dove tra l'altro Einaudi argomenta: «quel che erano allora gli operai che, attraverso a persecuzioni ed a carceri, capitanavano il movimento della loro classe, furono dal 1919 al 1921 i giovani ardenti che chiamarono gli italiani alla riscossa contro il bolscevismo». 
Non solo, ma viene altresì sottolineato come, là dove il socialismo ha trovato una pratica realizzazione, i risultati siano stati tragici sul fronte economico e anche rispetto ai diritti fondamentali ${ }^{33}$.

Due sono essenzialmente gli elementi di critica che Rosselli sviluppa in contrasto con le posizioni einaudiane, uno di natura teorica e un altro di ordine valutativo. La visione antagonistica e dinamica, propria del liberalismo, verrebbe pregiudicata dal vincolo ideologico. Il principio di un processo storico fondato su un continuo superamento dell'esistente, attraverso l'azione individuale e collettiva, trova un ostacolo insormontabile nella resistenza al mutamento di un determinato assetto economico. Emerge al riguardo quello che è stato definito come «il rifiuto del dottrinarismo aprioristico ${ }^{34}$, nel senso che la polemica con Einaudi esprimerebbe in modo significativo la ritrosia di Rosselli verso posizioni che muovono «da una premessa statica, conservatrice, nel racchiudere tutte le infinite possibilità di un domani anche lontano in una sorta di muraglia della Cina teoretica ${ }^{35}$. Di qui la critica più generale al liberalismo italiano di «generare la creatura e mozzarle le ali, dar vita a tutte le correnti progressive e rinnovatrici per poi negare ad esse, preventivamente, la facoltà, il diritto, financo la possibilità di superare la realtà in cui e da cui sorgono: in concreto la realtà capitalistica, borghese ${ }^{36}$. La pretesa che la teoria liberale non possa prescindere dalla tutela del sistema capitalistico pregiudica «la possibilità non solo di un governo diretto delle industrie da parte delle masse, ma anche della partecipazione degli operai e degli impiegati al governo dell'impresa ${ }^{37}$. In realtà, secondo Rosselli, è proprio in questa esigenza di emancipazione economica e morale che dovrebbe trovare espressione lo spirito liberale, altrimenti condannato alla negazione di se stesso ovvero al sacrificio del metodo in nome della salvaguardia del sistema. Questo è infatti il punto su cui Rosselli più insiste: la netta distinzione teorica tra metodo e sistema liberale, e contemporaneamente l'attribuzione al movimento operaio di una missione in sé liberale, in quanto emancipatrice. Non altro è il fondamento del secondo elemento della obiezione che Rosselli oppone al giudizio di Einaudi sulla lotta di classe come fonte di un socialismo illiberale sia nel suo

${ }^{33}$ Cfr. Ivi, pp. 109-128.

${ }^{34}$ G. Galasso, Politica e analisi economica nel pensiero di Carlo Rosselli, in Giustizia e libertà nella lotta antifascista e nella storia d'Italia, cit., p. 154.

${ }^{35}$ C. Rosselli, Luigi Einaudi e il movimento operaio, cit., p. 46-47.

${ }^{36}$ E Rosselli rileva una incongruenza nel «vedere nella storia un continuo divenire, una serie di equilibri successivi, una perpetua negazione dell'ieri e del domani, per poi isterilirsi in una dogmatica affermazione della perpetuità della realtà attuale» (ivi, p. 47). Riccardo Faucci ha colto negli appunti di Rosselli «parole efficaci nell'individuare uno dei punti deboli di una concezione del liberalismo come semplice complesso di regole del gioco immutabili, senza un principio interno di sviluppo, e soprattutto senza riconoscere la possibilità che il vincitore modifichi stabilmente le regole a suo favore» (R. Faucci, Einaudi, UTET, Torino 1986, p. 222).

${ }^{37}$ C. Rosselli, Luigi Einaudi e il movimento operaio, cit., p. 46. 
progetto sistemico (statalizzazione, collettivizzazione) sia negli strumenti atti a conseguirlo (violenza di classe). Nel passaggio dall'indagine dell'interrelazione liberalismo-capitalismo alla rivendicazione di un socialismo antistatalista e anticollettivista ${ }^{38}$, Rosselli associa la lotta di classe all'impegno per la realizzazione dell'autogoverno, quale espressione di una spinta progressista e di democratizzazione del mondo del lavoro:

[...] oggi le masse lavoratrici sono del tutto estranee alla direzione della produzione in cui domina un potere autocratico in violenta antitesi coi princìi democratici che, almeno formalmente, hanno trionfato nel campo politico [...] Ecco di nuovo il gigantesco problema, l'autogoverno dell' industria ${ }^{39}$.

È qui evidente la consapevolezza che l'intransigenza antisocialista dei liberali può essere depotenziata a patto di invalidare il nesso teorico e storico tra socialismo e collettivismo. A ben vedere, un'argomentazione simile era già stata formulata da Gobetti, proprio in polemica con Einaudi, per dimostrare che, avendo

[...] identificato il movimento operaio con le sue statiche formule collettivistiche, l'Einaudi lo ha discusso come una forma di socialismo di Stato. Ciò gli poteva essere consentito dall'esame di alcuni risultati empirici d'azione socialista, gli era contestato dallo spirito autonomistico e antiburocratico che presiede al risveglio operaio ${ }^{40}$.

Resta da vedere attraverso quali esperienze, in quali forme, e all'interno di quale progetto politico (e sicuramente qui le differenze con Gobetti non sono trascurabili) Rosselli svilupperà la trama di un socialismo che si fa interprete del metodo liberale, cercando di dimostrare come modificazioni del sistema economico rappresentino un passo avanti nella realizzazione delle istanze stesse del liberalismo.

\section{Liberalismo socialista}

La collaborazione con la rivista di Gobetti ha il suo momento di sintesi nella pubblicazione dell'articolo Liberalismo socialista, il 24 giugno del 1924; scritto che riprende, amplia e approfondisce un intervento dallo stesso tito-

${ }^{38}$ Cfr. Ivi, p. 48.

${ }^{39}$ Ivi, p. 51.

${ }^{40}$ P. Gobetti, Il liberalismo di Luigi Einaudi, «La Rivoluzione liberale», Anno I, N. 10, 23 aprile 1922, p. 38. Sul rapporto liberalismo-socialismo nel pensiero einaudiano Norberto Bobbio ha affermato che esso "corrispondeva, nella sua concezione di liberal-liberista, alla antitesi individualismo-statalismo, o addirittura, a quella ancora più netta, libertà-schiavitù» (N. Bobbio, Profilo ideologico del '900, Garzanti, Milano 1995, p. 109 (ed. orig. 1969)). 
lo sulla «Critica Sociale» del luglio $1923^{41}$. L'identità del titolo corrisponde alla continuità di un discorso in itinere. Con esplicito riferimento al corso delle vicende politiche, Rosselli procede nella messa a punto di una strategia teorica modulata su due fronti. Alla severità di giudizio verso l'indulgenza conservatrice del liberalismo italiano, fa da contrafforte il corpo a corpo con le contraddizioni attribuite alla classe dirigente socialista. Si viene così a delineare più compiutamente un'idea: la credibilità e l'efficacia della critica da sinistra alla spirale autoritaria dovrebbe derivare dalla definizione di una ipotesi riformista tanto radicale nei contenuti, quanto aderente ai cardini della liberaldemocrazia. Per Rosselli, l'impianto dottrinale cui paradossalmente il socialismo continua a tributare fedeltà, piuttosto che coincidere con un pilastro identitario irrinunciabile, è stato e continua a essere causa di aporie teoriche e divisioni politiche (dopo la scissione del gennaio 1921, nell'ottobre del 1922 un'ulteriore scissione nel fronte socialista aveva condotto alla costituzione del Partito Unitario di Turati e Treves).

In realtà, non si tratta di affrontare le pastoie di un'ulteriore revisione ideologica. Guardando al segmento minoritario che più ha incarnato nella storia del PSI l'incoerente convivenza di prassi gradualista e accettazione formale della dottrina rivoluzionaria, Rosselli disegna la traiettoria a suo avviso necessaria per uscire dal tunnel della sterilità teorica e politica, e rendere la teoria più liberale, ma non per questo la politica più moderata. Sul banco degli imputati c'è il marxismo, o meglio la versione positivistica del marxismo, che ha accompagnato il cammino del socialismo storico dalla fine dell'ottocento: una zavorra dell'azione politica. Effettivamente, Rosselli ritiene che un allontanamento dai dettami del marxismo si sia già realizzato nella prassi; le resistenze da abbattere riguardano il piano della "emancipazione" teorica. Questo discorso vale soprattutto per il socialismo riformista, l'unico, nelle sue diverse componenti, partitiche e sindacali, all'altezza "potenziale" del fine politico da realizzare.

Giudizio oscillante, quello relativo al riformismo, idealmente ancora "recuperabile", nonostante le riserve in sede di valutazione storica che spesso indurranno Rosselli a sferzate aspre. È innegabile però che per il momento il futuro leader di GL si senta parte, ancorché critica, di questa anima del socialismo, che dovrebbe

[...] non tanto rettificare la [...] posizione (già) profondamente mutata, quanto chiarirla, affermarla ancora più esplicitamente, senza timori di eresie, rompendo gli ultimi lacci, artificiosi che legano tuttora tanti fedelissimi militi dell'idea socialista alla lettera del pensiero marxista e a tutta la vecchia romantica fraseologia, ormai in contrasto stridente colla mutata realtà ${ }^{42}$.

${ }^{41}$ C. Rosselli, Liberalismo socialista, «Critica Sociale», 1-15 luglio 1923, ora in Id., Scritti politici, cit., pp. 55-60.

${ }^{42}$ Ivi, p. 56. 
Il fatto è che proprio l'impianto dogmatico da scardinare aveva costituito, attraverso l'opera di volgarizzazione, un motivo non secondario di radicamento. Tanto è vero che, dei tre partiti risultati dalla frammentazione del '21-'22, sarà proprio il meno connotato ideologicamente a ritrovarsi minoritario.

All'urgenza ultra-revisionistica contribuiscono diversi elementi. Lo spirito degli anni ' 10 aveva dato i suoi frutti: qualunque filosofia che odorasse di determinismo era in disgrazia agli occhi della nuova leva intellettuale. Al contempo, la predisposizione "costitutiva" ai valori liberaldemocratici impedisce a Rosselli di accostarsi al progetto gramsciano di recepire il volontarismo idealistico coniugandolo col Marx di Lenin.

La convinzione che l'ancoraggio al modello marxista avesse impedito lo sviluppo di un'idonea politica socialista, rafforzando altresì le ragioni della reazione contro il socialismo, trova conferma soprattutto nel tenore delle critiche mosse al massimalismo. La componente quasi sempre maggioritaria, che proclamava l'imminenza della rivoluzione senza farla, viene vista come mistura perversa di fatalismo ed estremismo. Amalgama che ha contagiato tutto il partito, sì che «in Italia non è mai esistito, dal 1900 in poi un Partito Socialista, che potesse dirsi veramente liberale e democratico» ${ }^{43}$. Il giudizio è tranchant e trascura ingenerosamente le conquiste di democrazia che tutto il movimento socialista - al di là della rappresentazione schematicamente dicotomica tra rivoluzionari e riformisti - era riuscito a ottenere nell'età giolittiana ${ }^{44}$. È evidente che in questa fase la recriminazione per l'impotenza socialista è indirizzata contro le conseguenze autolesionistiche dell'esasperazione ideologica.

Rosselli allora guarda al Partito Unitario. La formazione politica di Matteotti ha potenzialità effimere ${ }^{45}$. È un'esperienza emblematica nella storia di spaccature e diaspore del socialismo italiano. Nasce in un momento di naufragio e non può ambire a svolgere un ruolo né coesivo né egemonico ${ }^{46}$. Più che soffermarsi su questioni di strategia politica, la riflessione di Rosselli guarda a un orizzonte teorico che trascenda la contingenza. In un quadro diviso tra la convinzione di doversi preparare a una resistenza lunga e l'illusione che il fascismo si risolva in una parentesi, prevale la "sospensione" nell'(auto)chiarimento ideologico.

${ }^{43}$ Ibid.

${ }^{44}$ Ciò non toglie che l'azione politica socialista fosse indebolita non poco dal logorante dissidio tra correnti (cfr. A. Aquarone, Tre capitoli sull'Italia giolittiana, Il Mulino, Bologna 1987, p. 202).

${ }^{45} \mathrm{Cfr}$. Z. Ciuffoletti, Il PSU. Un partito ritardato o un partito mancato?, in F. Grassi Orsini e G. Quagliariello (a cura di), Il partito politico dalla Grande guerra al fascismo, Il Mulino, Bologna 1996, pp. 883-907.

${ }^{46} \mathrm{Nel}$ PSU non mancarono peraltro, anche se decisamente minoritarie, posizioni disponibili a un confronto collaborativo con il governo Mussolini tra il 1923 e il 1924, fino alla crisi Matteotti. Cfr. R. De Felice, Mussolini il fascista. La conquista del potere (1921-1925), Einaudi, Torino 1995, p. 610 e ss. 
Per Rosselli, la nuova formazione dovrebbe rappresentare un efficace centro di aggregazione dei riformisti, seguendo una "pratica socialista con metodo liberale» ${ }^{47}$. Il metodo liberale va insomma sempre distinto dal sistema liberale. Quest'ultimo «comporta regole e norme determinate [...] si richiama ad una specifica costituzione economico-sociale [...] e si riassume nell'ordinamento della società borghese ${ }^{48}$; il metodo significa invece «il rispetto per alcune fondamentali regole di giuoco, che stanno a base della civiltà moderna e che si riassumono nel sistema rappresentativo, nel riconoscimento di un diritto all'opposizione e nella ripulsa dei mezzi violenti ed illegali ${ }^{49}$.

In effetti, questa non è tanto una definizione teorica del metodo liberale, quanto un richiamo all'imprescindibilità di alcuni istituti del liberalismo politico. Rosselli fa risaltare la contraddizione in cui cade la maggior parte dei liberali, là dove rifiuta la possibilità di modificare l'ordine economico-sociale, pur nel rispetto del sistema politico. Contestualmente, auspica che venga ricomposta la scissione tra obiettivi concreti del socialismo e grammatica della liberaldemocrazia. Il sistema liberaldemocratico, se non può essere subordinato, o comunque correlato, ad alcun tipo di ordine economico, neppure può essere interpretato come un semplice strumento funzionale al raggiungimento della meta socialista. Viene così ribadito il rifiuto del miraggio palingenetico e sottolineato il valore in sé, non relativo, delle "regole del gioco".

Il primo principio che un partito socialista a metodo liberale deve acquisire è il rispetto verso «l'esistenza e lo sviluppo di altre ideologie e movimenti ad esso contrari» ${ }^{50}$. Non è in gioco la semplice rivendicazione del diritto all'opposizione, bensì il superamento teorico dell'orizzonte millenaristico, secondo l'affermazione che «un regime nel quale i contrasti di classe abbiano a scomparire e ad annegare completamente nell'armonia universale $\aleph^{51}$ non è auspicabile perché illiberale.

Ma il liberalismo per Rosselli non si limita a identificare un insieme di "buone" regole di funzionamento della società; rinvia a una ispirazione progressista, non «legata definitivamente a questo o quel gruppo, a questo o quel Partito $»^{52}$, ma alle forze che nella trama degli eventi storici si rendono protagoniste di conquiste di libertà ${ }^{53}$. Il riferimento alle vicende politiche italiane si fa esplicito. Nel momento in cui da più parti si abdica

${ }^{47}$ C. Rosselli, Liberalismo socialista (1923), cit., p. 57.

${ }^{48}$ Ibid.

${ }^{49}$ Ivi, p. 58.

${ }^{50}$ Ivi, p. 59.

${ }^{51}$ Ibid.

${ }^{52}$ Ibid.

${ }^{53}$ Cfr. P. Bagnoli, Liberalismo, socialismo, marxismo nel pensiero politico di Carlo Rosselli, in A. Bechelloni (a cura di), Carlo e Nello Rosselli e l'antifascismo europeo, FrancoAngeli, Milano 2001, pp. 29-36. 
alla funzione liberale e ci «si prosterna ai dittatori», «l'ultima trincea che rimane è la socialista $»^{54}$. Affiora qui chiaramente una doppia accezione del liberalismo. Il liberalismo coincide con un bagaglio di princìi e valori in teoria tendenzialmente formalizzati, che nel procedere della storia possono prevalere, essere minacciati o soccombere. Il liberalismo è però anche "imparentato" con una filosofia della storia progressiva; e di volta in volta vive negli individui o nei movimenti che si oppongono alla conservazione o alla reazione.

Esattamente un anno dopo, «La Rivoluzione liberale» pubblica la nuova versione dell'articolo di Rosselli dallo stesso titolo. Gobetti lo introduce con un commento di presentazione, che sottolinea l'interesse suscitato dalla riflessione critica di Rosselli all'interno della famiglia socialista e la comune attenzione per temi quali l'«autonomia economica e l'aspirazione delle masse ad affermarsi nella storia»o l'esame del «bilancio marxista $»^{55}$.

Tra il primo e il secondo Liberalismo socialista, le speranze che un'alleanza di centro-sinistra riuscisse a contrastare il fascismo erano sfumate. La classe dirigente liberale si era "suicidata" ${ }^{56}$. Le elezioni dell'aprile 1924 avevano conferito al Listone di Mussolini un'ampia maggioranza di seggi.

Rosselli riprende il filo del suo discorso e, di nuovo, ribadisce la distinzione del liberalismo in metodo e sistema. Su questa base delinea una sorta di classificazione delle diverse posizioni liberali presenti in Italia. Il registro è teorico, ma risente della delusione per la complicità o la cedevolezza di gran parte del liberalismo politico e intellettuale nei confronti del fascismo.

La denuncia rivolta al ceto dirigente è perentoria. Su di esso ricade la responsabilità di avere avallato il giro di vite autoritario, affidandosi a Mussolini, per allontanare il timore di rivolgimenti "rossi". Chi sostiene un'interpretazione del liberalismo vincolata ad un assetto economico e sociale cristallizzato, dovendo «scegliere tra metodo e sistema», è pronto a "sacrificare il primo al secondo». Sarebbe questo l'atteggiamento di «liberali marca Salandra $»^{57}$, che conduce a una profonda ostilità verso qualunque mutamento dell'esistente, risolvendosi quindi in un conservatorismo che, nei momenti di crisi sociale, si appella al principio di autorità incarnato nello Stato. In quest'ottica sarebbe stato accettato il fascismo, e con il fascismo lo stesso "sacrificio della libertà ${ }^{58}$. In realtà, dietro il richiamo al

${ }^{54}$ C. Rosselli, Liberalismo socialista (1923), cit., p. 60.

${ }^{55}$ C. Rosselli, Liberalismo socialista, «La Rivoluzione Liberale», 15 luglio 1924, con un commento introduttivo di Gobetti, ora in Id., Opere (vol. 1), cit., pp. 107128.

${ }^{56}$ Cfr. G. Sabbatucci, Il "suicidio" della classe dirigente liberale. La legge Acerbo 1923-1924, «Italia Contemporanea», n. 174, marzo 1989, pp. 57-80.

${ }^{57}$ C. Rosselli, Liberalismo socialista (1924), cit., p. 110.

${ }^{58}$ Ivi, p. 111. 
principio dì autorità, si nasconde la volontà di mantenimento dell'ordine costituito, a tutto vantaggio di quelle forze che detengono il potere nella società e nello Stato ${ }^{59}$.

Accanto a una concezione che, in definitiva, più che liberale, appare reazionaria, vi è quella dei sostenitori di un liberalismo classico, da Luigi Albertini a Einaudi. Essi esaltano la visione dinamica e antagonistica della storia, il cui continuo divenire comporta inevitabili rivolgimenti. Ma cercano di conciliare il metodo col sistema, ponendo così dei vincoli invalicabili all'azione riformista; con il risultato di «rispettare il metodo liberale solo nei confronti di chi, muovendosi all'interno del regime capitalistico con mentalità borghese, non mira a sovvertire il sistema» ${ }^{60}$.

Vi è infine un liberalismo relativistico, se non scettico, che assiste alle contraddittorie tendenze della realtà senza assumere posizioni critiche $o$ esprimere giudizi di valore. Ne farebbero parte personaggi come Prezzolini o Mario Missiroli, «spettatori [...] pronti ad inchinarsi a tutte le forze comunque scaturite, dovunque dirette ${ }^{61}$.

Queste sono, in estrema sintesi e con eccesso di semplificazione, le diverse anime del liberalismo italiano secondo Rosselli. Il quale si inserisce in un dibattito "strategico" per «La Rivoluzione Liberale». Cosa voleva dire essere liberali? Quale il compito politico da assolvere? Che atteggiamento tenere di fronte al fascismo? Rosselli non confida, come aveva fatto velleitariamente Augusto Monti, nell'avvento di un partito liberale di massa antifascista, scollato da democratici e socialisti ${ }^{62}$. E prova una insofferenza venata di moralismo per la soluzione degli Apoti proposta da Prezzolini ${ }^{63}$.

${ }^{59}$ Le considerazioni di Rosselli riecheggiano quelle contenute in un articolo di Carlo Levi. Salandra era «sopra tutto e innanzi tutto conservatore; per indole, per istinto, per posizione sociale», che intendeva lo Stato come "pura organizzazione centrale» e problema di "autorità», da opporre al rischio di eversione della classe operaia (C. Levi, Antonio Salandra, «La Rivoluzione Liberale», Anno I, N. 25, 27 agosto 1922, pp. 91-92).

${ }^{60}$ C. Rosselli, Liberalismo socialista (1924), cit., p. 113.

${ }^{61}$ Ivi, p. 115.

${ }^{62}$ Richiamandosi alle posizioni del direttore del «Corriere della Sera» Luigi Albertini, Monti aveva indicato l'opportunità di impegnarsi nella creazione di un partito liberale che, recuperando l'ispirazione del combattentismo à la Salvemini, fosse in grado di contrapporsi al fascismo senza "accordarsi a nessuna democrazia». A margine dell'intervento di Monti, Gobetti postillava con una stoccata, tesa a mettere in luce le contraddizioni di liberali come Albertini: «ma perché si possa discorrere di azione liberale Luigi Albertini deve rispondere al nostro dilemma: o accettare la lotta di classe e chiamare gli operai al mito libertario o accontentarsi di fascismo» (Postilla a A. Monti, Il liberalismo e le masse, "La Rivoluzione Liberale» Anno II, N. 9, 10 aprile 1923, p. 37).

${ }^{63}$ G. Prezzolini, Per una Società degli Apoti, «La Rivoluzione Liberale», Anno I, N. 28, 28 settembre 1922, pp. 103-104. Con un commento di Gobetti, che prendeva le distanze dall'idea di un ritiro dell'intellettuale dalla mischia politica, in cui la 
Liberalismo socialista non si limita alla panoramica polemica; offre anche un impegno costruttivo. L'ideologia liberale si è affermata storicamente attraverso le forze sociali borghesi, che hanno visto così riconosciuti diritti e sanciti poteri. Ma, se il dispiegamento progressivo della libertà rappresenta lo spirito della dottrina liberale, non si possono escludere le forze che se ne rendono nuovi interpreti storici. Rosselli non ha dubbi nell'individuare nelle classi subalterne i protagonisti della funzione liberale contemporanea. Con che cosa, più specificamente, si identifica questa funzione? La lotta per la libertà equivale alla lotta per l'acquisizione di diritti. L'allargamento alle masse dei diritti politici, del diritto di controllo sullo Stato, diviene pertanto elemento imprescindibile di progresso civile, attraverso cui liberalismo e democrazia si fondono, poiché «la seconda non è che il logico sviluppo del primo, e il primo non sussiste sostanzialmente in uno Stato rappresentativo moderno senza la seconda ${ }^{64}$. Non altro è il criterio di identificazione delle forze politiche e sociali su cui Rosselli confida. Perché quale «più grande funzione liberale, liberatrice, di quella che si concreta nel movimento di sindacati, cooperative, partiti, che sinteticamente chiamiamo socialisti? ${ }^{65}$. Sono questi i soggetti che svolgono un ruolo paragonabile a quello della borghesia nel sistema assolutista; sono infatti «le minoranze, le opposizioni, i gruppi ancor deboli, bisognosi per ragioni fisiologiche di un'atmosfera di libertà e di autonomia che assicuri loro la possibilità di sviluppo, le vere forze liberali ${ }^{66}$.

Certo, una democrazia che miri al reale riscatto delle masse non può fondarsi esclusivamente sul principio giuridico delle libertà negative; non ci si può fermare al neminem laedere. Il riscatto è strettamente collegato alla rivendicazione di diritti economici poiché,

[...] mentre per la borghesia la concessione e la conquista delle libertà politiche costituì la sublimazione, il coronamento della sua potenza già affermatasi in sede economica e culturale, per il proletariato rap-

riflessione lucida e disinteressata era impossibile. Ma per la lunga e travagliata polemica tra Gobetti e Prezzolini, cfr. G. Prezzolini (a cura di), Gobetti e "La Voce", Sansoni, Firenze 1971.

${ }^{64}$ C. Rosselli, Liberalismo socialista (1924), cit., p. 117.

${ }^{65}$ Ivi, p. 119.

${ }^{66} \mathrm{Ibid}$. Silvio Suppa ha visto in Rosselli una concezione liberale in cui «il nesso fra liberalismo e progresso ne attiva un altro fra liberalismo e movimento, cosicché la continuità liberale della fase illuministico-rivoluzionaria, al presente, risulta tutta nella coerenza di una coscienza indomita e di un'accezione non conformistica del valore della libertà». Il patrimonio al quale Rosselli sembra rivolgersi «è proprio quello della libertà dei moderni, rivissuto contro ogni continuismo istituzionale» (S. Suppa, Note su Carlo Rosselli: temi per due tradizioni, in M. Bovero, V. Mura, F. Sbarberi, (a cura di), I dilemmi del liberalsocialismo, La Nuova Italia Scientifica, Roma 1994, pp. 194-95). 
presentò se non l'inizio della lotta per l'emancipazione, la premessa, lo strumento per più rapidamente conseguirla ${ }^{67}$.

Lettura, questa, che trova una sponda nelle considerazioni espresse sempre sulla rivista di Gobetti da un liberale come Guido De Ruggiero, il quale, pur valutando i rischi di una deriva "dispotica" della democrazia, affermava che la libertà e l'eguaglianza innanzi alla legge non bastano al piccolo borghese e all'operaio. Essi

[...] hanno sempre trovato abbastanza ironico questo concetto della legalità: che c'importa, essi hanno detto, l'eguaglianza e la libertà innanzi alla legge, se non abbiamo modo di liberare la nostra sorte? Senza contare che è un'uguaglianza molto discutibile, anche innanzi alla legge, quella del ricco e del povero, dell'operaio isolato e del capitalista ${ }^{68}$.

Resta il problema della via (o meglio della condizione) più idonea al conseguimento della cosiddetta "autonomia economica". Non è certo attraverso una semplice opera legislativa di redistribuzione del reddito che si può giungere a un effettivo innalzamento materiale e morale delle classi lavoratrici, "come ritengono i liberali ortodossi, attraverso il parlamento politico ${ }^{69}$. Allo stesso tempo Rosselli prende però radicalmente le distanze dal socialismo statalista e collettivista, che «le recenti esperienze, tutte le esperienze di questi ultimi trent'anni hanno condannato senza speranza ${ }^{70}$. Vengono al riguardo individuati in modo chiaro $\mathrm{i}$ "pericoli enormi della burocrazia, della incompetenza, della invadenza statale, dello schiacciamento della libertà individuale $»^{71}$. E con tale pericolo trova conferma una esplicita vocazione antistatalista, la diffidenza verso uno Stato sempre in sé pericolosamente pervasivo, opprimente, che va contenuto in quanto limita l'autonomia individuale e finisce per divenire strumento di dominio, sia nella versione borghese che in quella collettivistica ${ }^{72}$. Matu-

${ }^{67}$ C. Rosselli, Liberalismo socialista (1924), cit., p. 120.

${ }^{68}$ G. De Ruggiero, Il liberalismo e le masse, «La Rivoluzione liberale», Anno II, N. 12, 1 maggio 1923, p. 49. In questo articolo De Ruggiero, riprendeva una sua lettura "dialettica" della borghesia, classe sociale che era riuscita a farsi interprete delle libertà moderne, ma che si era ritrovata, una volta consolidatosi il regime capitalistico di proprietà, a veder confliggere i propri interessi con i propri valori. Pur non riconoscendosi nella visione materialistica della storia, De Ruggiero considerava realisticamente inevitabile il prodursi di un fenomeno del genere (G. De Ruggiero, I presupposti economici del liberalismo, «La Rivoluzione Liberale», Anno I, N. 2, 19 febbraio 1922, pp. 6-7).

${ }^{69}$ C. Rosselli, Liberalismo socialista (1924), cit., p. 120.

${ }^{70}$ Ivi, p. 124.

${ }^{71}$ Ivi, p. 125.

${ }^{72}$ Cfr. Z. Ciuffoletti, Contro lo statalismo, Lacaita, Manduria 1999, specialmente il primo capitolo, dal titolo "Più o meno Stato". La critica rosselliana al centralismo e allo statalismo, pp. 11-42. 
ra invece in Rosselli l'adesione a un modello sindacalistico-cooperativo ovvero il riferimento al tema del controllo operaio, per cui un processo di effettiva crescita democratica deve muovere

[...] non dall'alto al basso, non dal centro alla periferia, ma all'inverso. Il socialismo in tutti i suoi aspetti non ha da essere frutto di imposizione, ma di conquista, anzi di autoconquista [...] deve sprigionarsi naturalmente dallo stesso moto operaio, dalle esperienze infinite delle leghe, delle cooperative, delle istituzioni culturali ${ }^{73}$.

Questa è la risposta a chi, come Riccardo Bauer, affermava, proprio dalle colonne della «Rivoluzione liberale», che «ogni formazione socialista si riduce sempre alla creazione di un ordine accentrato regolatore dei fatti economici, si riduce sostanzialmente alla applicazione di un principio statolatra $»^{74}$.

Proprio in rapporto ai nuovi obbiettivi da conseguire, è necessario precisare il metodo e i criteri che devono guidare l'azione delle masse proletarie. Secondo Rosselli è fondamentale l'acquisizione di un abito mentale, di una cultura realmente liberale, poiché essa non è «né borghese, né socialista. É un minimo di civiltà che tutti, per interesse prima personale, e poi umano, si impegnano di rispettare $»^{75}$. Ciò non vuol dire che, di fronte a un atteggiamento autoritario o repressivo, il proletariato non abbia diritto a reagire. Se è vero che la legalità è un valore che non ha colore ideologico, è altrettanto vero che la sospensione della legalità, finalizzata a contenere o impedire il mutamento sociale, legittima il diritto di resistenza, il ricorso a una «sacrosanta e liberalissima violenza $»^{76}$. E tuttavia la violenza non può essere prevista o giustificata dottrinalmente come strumento per la conquista del potere, in un contesto di piena legalità liberale; in esso al socialismo si impone una strategia d'azione fatta di riforme e non di rivoluzione. Va da sé che la chiarezza sul metodo getta luce sulla stessa identità di un fine, che è tale per Rosselli in quanto non rappresenta un modello organico né si realizza per l'obbligo della necessità storica; il socialismo è «un ideale [...] che induce a superare di continuo la posizione acquisita conforme all'elemento dinamico progressista dei ceti inferiori che salgono irresistibilmente. Ora lo stato d'animo liberale non sta anche in questa sete indefinita di progresso, di superamento, in questo ideale di marcia eterna? $\rangle^{77}$.

Se conviene prescindere da una prospettiva di palingenesi sociale, non è solo perché essa si fonda su una previsione errata; è soprattutto l'idea

${ }^{73}$ C. Rosselli, Liberalismo socialista (1924), cit., p. 126.

${ }^{74}$ R. Bauer, Domande ai socialisti, «La Rivoluzione Liberale», Anno III, N. 26, 24 giugno 1924, p. 101.

${ }^{75}$ C. Rosselli, Liberalismo socialista (1924), cit., p. 122.

${ }^{76}$ Ivi, pp. 122-123.

${ }^{77}$ Ivi, p. 124. 
stessa di "fine della storia" che risulta a Rosselli affatto ingiustificata, in contrasto con chi ritiene impossibile per i socialisti abbracciare coerentemente il metodo liberale, in quanto costitutivo del socialismo sarebbe l'obiettivo di realizzare un «novus ordo [...] capace di attuare lo stato di perfetta giustizia sociale vagheggiato ${ }^{78}$, quindi un "sistema-altro" statico e definitivo, sostitutivo del sistema capitalistico. L'animo liberale di un socialista si manifesta in un approccio critico, relativista, empirista, che non sminuisce la convinzione in un ideale abbracciato anche per «fede e per sentimento ${ }^{79}$. È questa una linea interpretativa che avvicina Rosselli alle considerazioni espresse da Alessandro Levi in un articolo dal titolo Liberalismo come stato d'animo, là dove è messo in chiaro che il metodo liberale si caratterizza per il riconoscimento dei «limiti giuridici imposti alle attività per necessità sociali», concependo la «lotta civile» come «una libera concorrenza fra le varie fedi ${ }^{80}$. In questo senso lo stato d'animo liberale si oppone "come a sua netta antitesi, ad un altro stato d'animo [...] e cioè al settarismo od intolleranza»" appartiene a una «aristocrazia morale, che richiede rarissime virtù di pensiero e di azione ${ }^{82}$, per Rosselli è possibile e doveroso coinvolgere le masse in un processo di acquisizione culturale di questi valori.

Si è detto dell'interesse di Gobetti per lo sforzo critico dell'analisi rosselliana. Il confronto con un socialista attento al profilo autonomistico della democrazia dal basso e scevro da connotazioni stataliste, trovava immediata disponibilità nel direttore di «La Rivoluzione Liberale». Ciò non esclude la necessità di sottolineare evidenti differenze, sia di giudizio che di proposta politica. Se entrambi tentano di individuare la via che consenta alle masse di emergere, contrastanti sono le valutazioni sull'azione storica del socialismo italiano e sugli strumenti più efficaci con cui avviare il cambiamento sociale e politico. Come si è visto, Rosselli esprime critiche molto severe rispetto all'operato del partito socialista nel primo ventennio del secolo; ostile al massimalismo, non lesina attacchi anche alla componente riformista, di cui però si sente parte, fino al punto da iscriversi al partito unitario, dopo l'uccisione di Matteotti. Decide, nella fase più drammatica e decisiva, di impegnarsi "dal di dentro", cercando di risolvere "l'equivoco" marxista, ed esprimendo una posizione di volontarismo e intransigenza morale.

Del tutto estraneo al socialismo riformista è Gobetti. Duro il suo giudizio sul riformismo turatiano, reo di una politica contigua al giolittismo e spesso sfociante nella ricerca del privilegio parassitario. In sede di valutazione storica, Gobetti ripropone le accuse di Salvemini e di Einaudi:

${ }^{78}$ R. Bauer, Domande ai socialisti, cit., p. 101.

${ }^{79}$ C. Rosselli, Liberalismo socialista (1924), cit., p. 127.

${ }^{80}$ A. Levi, Liberalismo come stato d'animo, «La Rivoluzione Liberale», Anno II, N. 17, 5 giugno 1923, p. 72 .

${ }^{81}$ Ibid.

${ }^{82}$ Ibid. 
[...] le prime aristocrazie operaie, invece di mantenere le loro posizioni di intransigenza, invocano borghesemente la protezione della legislazione sociale, come le timide iniziative industriali chiedono l'appoggio del protezionismo doganale e delle sovvenzioni governative ${ }^{83}$.

Giusto un'apertura risalente alla crisi di fine secolo. Se al tempo di «Crispi e Pelloux, Turati seppe condurre la battaglia con singolare arte diplomatica e con grande generosità», un atteggiamento gradualistico e compromissorio esaurì di lì a poco «tutta l'originalità di pensiero del nostro socialismo» ${ }^{84}$. Con il risultato che, dopo la guerra,

[...] appena il popolo ebbe coscienza di esser rimasto esterno alla formazione nazionale, guidato per venti anni dai riformisti a un'opera anarchica di sfruttamento dello Stato, e volle una sua disciplina sovvertitrice di un ordine impostogli da tradizioni non sue, Turati si trovò a parlare attraverso i fiori della retorica messianica un linguaggio reazionario ${ }^{85}$.

I «fiori della retorica messianica» sono la metafora del disarmo, dell'abbandono del radicalismo combattivo, irreversibilmente espulso dalla cultura riformista. E mai più riconquistato, né riconquistabile. A differenza di Rosselli, difatti, Gobetti giudica la nascita del PSU quasi alla stregua di un puntello «collaborazionista» al governo fascista ${ }^{86}$, ed è lontano dall'intravedere nel riformismo la formula adatta a coagulare un fronte politico antifascista.

Una simile chiave di lettura non lascia a Gobetti spazio di interesse per un'opera revisionistica che conduca a un socialismo liberale, liberato$r e$, da parte di un partito che ritiene legato a una pratica antivolontarista e conciliativa. A questa divergenza di giudizio consegue l'individuazione di strategie politiche differenti. Per Rosselli una pratica riformista e democratica può favorire l'emancipazione del lavoro, con il contributo di un partito socialista a metodo liberale. Sono, appunto, i sindacati, le cooperative, e le forze del socialismo riformista a poter condurre la lotta per il

${ }^{83}$ P. Gobetti, La Rivoluzione Liberale. Saggio sulla politica in Italia, cit., p. 27.

${ }^{84}$ Ivi, p. 87.

${ }^{85}$ Ivi, p. 88.

${ }^{86}$ «Il blocco antifascista costituito intorno al Partito Socialista Unitario sarà un blocco collaborazionista», così Gobetti in Gli unitari a Convegno, «La Rivoluzione Liberale», Anno II, N. 36, 20 novembre 1923, p. 145. E continuava accomunando, sotto il profilo della cultura profonda, fascismo e socialismo, entrambi connotati da spirito opportunistico, cui Gobetti contrapponeva la sua "mistica" dell'«intransigenza»: «I socialisti unitari sono pronti a diventare sempre più evangelici. Il loro sogno lontano è identico con quello di Mussolini, un'Italia pacifica, senza partiti, patriarcale, unanime, nelle mani oneste e gentili dei mandarini sindacali». Sull'antiriformismo di Gobetti, cfr. M. Gervasoni, L'intellettuale come eroe. Piero Gobettie le culture del Novecento, La Nuova Italia, Firenze 2000, pp. 293 e ss. 
progresso democratico, sia in politica (suffragio universale e partecipazione alle scelte della legislazione) che in economia (autogestione, iniziativa dal basso). Gobetti guarda invece con interesse all'esperienza gramsciana dei Consigli di fabbrica ${ }^{87}$, giudicando decisiva l'azione di una minoranza cosciente, capace di creare nuclei autonomi di produttori, non irretiti dal burocratismo partitico o sindacale. Poiché il

[...] sindacato è organo di resistenza, non di iniziativa, tende a dare all'operaio la sua coscienza di salariato, non la dignità del produttore [...] nel Consiglio invece l'operaio sente la sua dignità di elemento indispensabile della vita moderna [...] pone al centro delle sue aspirazioni non il pensiero dell'utile particolare, ma un ideale di progresso e di autonomia [...] e cerca di fondare un'organizzazione pratica attraverso la quale la sua classe conquisti il potere ${ }^{88}$.

Se l'esperimento ordinovista è fallito per «l'impossibilità di estendere il movimento a tutta l'Italia sia per gli ostacoli economici, sia per l'inesistenza fuori di Torino di una classe dirigente operaia matura», Gobetti crede comunque che chi,

[...] fuori di ogni pregiudizio di partito, pensoso degli effetti della crisi postbellica che è crisi di volontà, di coerenza, di libertà, confidi ancora nella ripresa del movimento rivoluzionario interrotto nel Risorgimento [...] dovrebbe scorgere in questi sentimenti e in queste prove la via maestra della lotta politica futura ${ }^{89}$.

Da questo confronto di idee emerge quindi un modo differente, anche se non inconciliabile, di concepire la lotta di libertà e autonomia della classe lavoratrice. Da un lato, il socialismo eretico di Rosselli, visione anti-ideologica, di progressismo democratico; dall'altro, l'eresia liberale di Gobetti, rivoluzionaria, avanguardista, eroica, in definitiva «liberale perché conflittuale, anziché conflittuale perché liberale» ${ }^{90}$.

${ }^{87}$ Gobetti offrì un giudizio favorevole alla scissione dei comunisti a Livorno, proprio a partire dalla valutazione positiva della componente ordinovista: «i veri rivoluzionari italiani non potevano più aver fede nel Partito Socialista, diventato partito di maggioranza, incapace d'azione per l'elefantiasi burocratica del suo ordinamento, per il pregiudizio dell'unità, per le iniziali responsabilità di governo» ( $\mathrm{P}$. Gobetti, Storia dei comunisti torinesi, scritta da un liberale, "La Rivoluzione Liberale», Anno I, n. 7, 2 aprile 1922, p. 26).

${ }^{88}$ P. Gobetti, La Rivoluzione Liberale. Saggio sulla politica in Italia, cit., p. 103.

${ }^{89}$ Ivi, pp. $104-105$.

${ }^{90}$ M. Revelli, Gobetti "liberal-comunista"?, in M. Bovero, V. Mura, F. Sbarberi (a cura di), I dilemmi del liberalsocialismo, cit., p. 83. Sui rapporti tra socialismo e liberalismo in Gobetti si vedano le pp. 78-84. 


\section{CAPITOLO TERZO}

\section{UNA PROPOSTA SOCIALISTA}

\section{Socialismo e marxismo}

Il socialismo riformista aveva subito la spaccatura dell'ottobre 1922. Il congresso che ne sanciva l'espulsione, produceva il collasso dello spirito unitario, al quale soprattutto Turati aveva tentato fino all'ultimo di appellarsi ${ }^{1}$. La scissione era una soluzione obbligata all'incompatibilità con la maggioranza del partito. La nascita del PSU ratificava la frantumazione politica del socialismo italiano. Il massimalismo non era riuscito a catalizzare, su una linea rivoluzionaria convincente e condivisa, né l'ala comunista, né quella turatiana. Il PCd'I avrebbe tentato di perseguire, pur con notevoli fibrillazioni interne, una via italiana al comunismo ${ }^{2}$. Per i riformisti, la solitudine poteva significare uno smacco o un'occasione. La separazione si produsse in concomitanza con l'ascesa al governo di Mussolini. Turati, nonostante i ripetuti tentennamenti, non aveva mai sfidato il gruppo parlamentare a oltrepassare i confini dell'opposizione. E questa ipotesi, probabilmente impraticabile, non si realizzerà mai ${ }^{3}$. Pur aderen-

${ }^{1}$ Nel suo intervento al XIX Congresso, Turati aveva affermato: «Noi ci separiamo da voi: $o$, forse più esattamente (non vi sembri una sottigliezza), voi vi separate da noi. Comunque ci separiamo. Accettiamo l'esito della votazione». Aggiungendo: "Accomiatiamoci al grido augurale di viva il socialismo! Auspicando che questo grido possa un giorno - se sapremo esser saggi - riunirci ancora una volta in un'opera comune di dovere, di sacrificio, di vittoria!» (F. Turati, Echi del Congresso socialista. Il discorso di commiato di Filippo Turati pei socialisti unitari, «Critica Sociale», Anno XXXII, N. 20, 16-31 ottobre 1922, pp. 308-311).

${ }^{2}$ Cfr. F. Vander, Livorno 1921. Come e perché nasce un partito, Lacaita, Manduria-Bari-Roma 2008. La chiave interpretativa di questo volume insiste molto sulla natura endogena del progetto comunista. Pur non sottovalutando l'influenza del mito bolscevico, Vander attribuisce al gruppo di Gramsci, Togliatti e Terracini, che sarebbe riuscito a imporsi nel 1926 a guida del partito, un disegno politico inserito nella storia nazionale.

${ }^{3}$ Cfr. L'epistolario tra Filippo Turati e Anna Kuliscioff dal maggio 1920 all'ottobre 1922, in F. Turati, A. Kuliscioff, Carteggio. V. dopoguerra e fascismo (1919-22), Einaudi, Torino 1953. Sulla posizione di Turati, cfr. M.L. Salvadori, La sinistra nella storia d'Italia, Laterza, Roma-Bari 1999, pp. 57-58. 
do al PSU soltanto dopo l'attentato Matteotti, Rosselli è vicino al nuovo partito ${ }^{4}$. Ma è una vicinanza declinata in termini di contributo critico. La creazione di un soggetto socialista riformista, per quanto minoritario, poteva avere senso solo a patto di una revisione profonda degli equivoci ideologici che per lungo tempo erano corrisposti al prezzo della convivenza unitaria. La vecchia classe dirigente, da Turati a Treves e Modigliani, aveva sempre proceduto sul crinale tra riforme e rivoluzione. Ladesione al pragmatismo evolutivo del programma minimo conviveva con la dichiarazione di fede nell'avvento della società armonica senza classi; la mentalità dei piccoli passi, supportata dalla fiducia positivista nel necessario e graduale sviluppo verso il socialismo, non comportava l'abbandono del lessico messianico.

I nodi da sciogliere riguardavano dunque l'intreccio tra visione politica e codice culturale di riferimento. Più in profondità, secondo Rosselli tutto il socialismo italiano doveva almeno in parte la sua sconfitta a una "tara" filosofica: il marxismo. O meglio, all'identificazione, per quanto sempre più rituale, delle sorti del movimento socialista con la teleologia della dottrina di Marx.

All'interno della cultura riformista, l'interlocutore naturale con cui confrontarsi sul nesso tra socialismo e marxismo era Rodolfo Mondolfo. Il filosofo marchigiano aveva elaborato una approfondita esegesi, confluita nella raccolta di saggi dal titolo Sulle orme di Marx, che conobbe tre edizioni in cinque anni ${ }^{5}$. Questa sintesi costituiva il tentativo più serio e rigoroso - successivo ad Antonio Labriola e precedente ad Antonio Gramsci - di conferire al socialismo italiano una filosofia organica. Mondolfo voleva assolvere il compito di unificare le varie articolazioni politiche del socialismo sotto un unico ombrello ideologico. Il suo Marx "umanistico" ambiva a essere la formula a egemonia riformista in grado di sancire l'identità tra socialismo e marxismo e l'unità culturale del movimento operaio. La prospettiva mondofiana corrispondeva a una proposta "mediatrice". Sul piano squisitamente filosofico risolveva il dilemma tra volontarismo e determinismo attraverso la chiave epistemologica del rovesciamento della praxis, che conciliava dialetticamente libertà e necessità; sul piano della filosofia della storia, individuava nel marxismo la Weltanschauung che sussumeva - superando senza negare - il liberalismo e la democrazia; sul piano più propriamente politico, individuava nella continuità delle riforme la via per realizzare la rivoluzione e nella

${ }^{4}$ In occasione delle elezioni amministrative a Milano del dicembre 1922, vinte dal Blocco composto da fascisti, liberali e popolari, Rosselli è confortato dal risultato dei riformisti: «Io sono tutto riconsolato per l'affermazione buonissima degli unitari; bisogna tenere presente che per il blocco hanno votato anche i sedicimila popolari che si astennero nel 1920» (lettera alla madre del 13 dicembre 1922, in Epistolario familiare, cit., p. 135).

${ }^{5}$ Tutte e tre le edizioni furono pubblicate dall'editore Cappelli di Bologna, nel 1919, 1920 e 1923, quest'ultima notevolmente accresciuta. 
lotta di classe lo strumento non cruento per giungere a una società olisticamente socialista ${ }^{6}$.

Ma mediazione, continuità, compromesso non erano termini capaci di attecchire nel contesto conflittuale e destabilizzato del dopoguerra. La versione socialdemocratica del marxismo era entrata in crisi irreversibile con la guerra e i tentativi di rianimarla, sia pure con gli accorgimenti filosofici di Mondolfo, erano destinati a fallire. La generazione della guerra o non si riconosceva in Marx o lo leggeva con uno spirito di rottura. Da questo punto di vista, La rivoluzione contro il Capitale di Gramsci e L'ora di Marx di Gobetti assumono valenza esemplare quasi iconica.

Come si è accennato, l'obiettivo di Rosselli non è promuovere un'ennesima declinazione dell'opera di Marx, ma piuttosto confutare il rapporto di dipendenza dell'azione socialista dal marxismo. Nel novembre del 1923, «Critica sociale» pubblica l'articolo dal titolo La crisi intellettuale del partito socialista ${ }^{7}$, che determina l'inizio del confronto con Mondolfo sulla funzione del sistema marxista quale base ideologica del socialismo ${ }^{8}$. Il punto di partenza dello scritto è una constatazione critica: la rivista storica del riformismo ha visto l'affievolirsi progressivo del dibattito culturale e della capacità di stimolo intellettuale. In realtà, si tratta di un'accusa rivolta all'intera intellighenzia socialista. Rosselli ritiene che il perdurante rimasticamento del linguaggio liturgico del marxismo non abbia significato la salvaguardia di una teoria coerente, funzionale a una efficace filosofia dell'azione. Ha prodotto invece l'ancoraggio a un armamentario dogmatico contraddittorio e fossilizzante. Contraddittorio, in quanto la prassi si è spesso discostata da dettami teorici sempre più indefiniti e generici; fossilizzante, perché non è stato dato spazio a proposte critiche innovative.

Esaminando il marxismo da un punto di vista della validità teorica, salta agli occhi un'opera spasmodica di «interpretazioni e riduzioni» tale che «quella dottrina [...] a forza di venir corretta, annacquata, adulterata [...] finì per trasformarsi in qualche cosa di così vago ed incerto da poter ad un tempo servire ad ogni frazione ${ }^{9}$. Il marxismo appare così un magma indistinto, il quale pretende adesione per atto di fede, piuttosto che per verifica del fondamento di verità e possibilità di applicazione delle sue diverse tesi. Converrebbe perciò, in via preliminare,

[...] eseguire un vero e proprio bilancio teorico della dottrina marxista che, partendo da basi essenzialmente scientifiche e realistiche, collo scartare cioè tutto ciò che è in contraddizione coi fatti, o in contrad-

${ }^{6}$ Per tutti questi aspetti, rinvio ancora a C. Calabrò, Il socialismo mite, cit.

${ }^{7}$ C. Rosselli, La crisi intellettuale del partito socialista, «Critica sociale», 1-15 novembre 1923, ora in Id., Opere (vol. 1), cit., pp. 83-95.

${ }^{8}$ Cfr. R. Treves, Rodolfo Mondolfo e i fratelli Rosselli, "Critica sociale», supplemento al N. 24, 20 dicembre 1967, pp. 12-14.

${ }^{9}$ C. Rosselli, La crisi intellettuale del partito socialista, cit. p. 84. 
dizione col generico indirizzo del partito e del movimento socialista, ci dicesse ciò che è vivo e ciò che è morto del marxismo ${ }^{10}$.

Rispetto a tale bilancio, Rosselli è perentorio nell'individuare, all'interno del ginepraio teorico marxista, due elementi da "salvare": materialismo storico e lotta di classe. Il punto è che questi due «capisaldi» devono essere considerati come criteri storico-interpretativi, non come principi di sostegno all'azione del movimento operaio. Ne consegue che «non è detto che oggi l'essere marxisti voglia dire essere socialisti. Il fatto che scrittori conservatori come il Pareto [...] abbiano potuto accettare questa parte della dottrina marxista, conferma a chiare note che si può essere marxisti senza essere socialisti» ${ }^{11}$. In realtà, l'obiettivo rosselliano non è una revisione marxista, ma un superamento del marxismo, fardello ideologico ingombrante, che rende angusti gli spazi di analisi critica e opera da freno rispetto all'efficacia della prassi. Risalta così la visione volontarista e insieme pragmatica di Rosselli. Il marxismo viene visto come un macigno sulla strada dell'evoluzione del socialismo riformista in senso democratico e liberale.

Il problema non è solo dottrinario, ma politico. La prospettiva rosselliana si rivolge all'affermazione di un movimento partitico flessibile, aperto e, per così dire, deideologizzato; e incoraggia quindi

[...] l'abbandono di questa tendenza dogmatica del partito, di questa spesso inconscia ma continua subordinazione dell'azione concreta d'un movimento di masse ad una rigida teoria. Un partito ha bisogno di un grado estremo di elasticità, di una grande libertà di atteggiamenti, anche se è necessario che mantenga una chiara e coerente linea di condotta nel tempo. Un partito legato ad un corpo rigido di dottrine finisce per appesantirsi, per muoversi con una lentezza esasperante ${ }^{12}$.

Oggetto di valutazione non è qui l'analisi teorica, la fondatezza o meno degli assunti marxisti. Rosselli afferma piuttosto un'esigenza di «sano empirismo all'inglese ${ }^{13}$. Sulle sue considerazioni pesa il giudizio rispetto ai limiti della politica socialista nel primo ventennio del secolo, per cui nell'atteggiamento «di molti socialisti [...] era troppo chiara l'influenza di quel fatalismo cosiddetto marxista, che deriva da una erronea, per quanto spiegabilissima, interpretazione degli scritti più conosciuti di MarX» ${ }^{14}$. Rosselli sottolinea come la traduzione del marxismo in uno specifico atteggiamento politico ha fatto sì che

\footnotetext{
${ }^{10}$ Ivi, p. 85.

${ }^{11}$ Ivi, p. 87.

${ }^{12}$ Ivi, p. 89.

${ }^{13}$ Ivi, p. 88.

${ }^{14}$ Ivi, p. 90.
} 
[...] problemi vitali che occorreva esaminare e risolvere con spirito realistico, strettamente adeguando l'azione del partito a quelli che sono i concreti bisogni di una particolare collettività in un determinato momento storico, finirono per essere regolarmente trascurati, o semplicisticamente esaminati e risolti alla luce esclusiva dei princìi marxistici ${ }^{15}$.

E il riferimento riguarda non solo l'impegno su temi quali il suffragio universale, l'istruzione, il sindacato, il Mezzogiorno; già adesso è messa in rilievo, in modo esplicito, anche l'inerzia rispetto all'avvento del fascismo. Un atteggiamento complessivo quindi ingessato, che non avrebbe consentito al socialismo di svolgere una funzione di efficace "spinta propulsiva", di rinnovamento e coinvolgimento di forze nuove.

Quest'ultimo è un punto su cui Rosselli insiste particolarmente, riferendosi soprattutto alla sua generazione, vittima del disorientamento postbellico e la cui tensione attivistica non è stata compresa efficacemente dai socialisti:

[...] i giovani ebbero l'impressione che l'ingresso nel partito significasse indossare una terribile cappa di piombo annichilente ogni personalità, una preventiva rinunzia a qualunque libertà spirituale, il divieto di orientarsi verso direzioni nuove. L'imposizione, in una parola, di un ritmo obbligato di pensiero e di azione ${ }^{16}$.

Rosselli indica quindi una strategia di apertura a istanze provenienti da ambienti democratici e salveminiani, per contrastare la tendenza all'isolamento e al sacrificio di importanti energie sociali e politiche. Si tratta anche in questo caso del richiamo al metodo liberale, nella convinzione che l'autonomia del movimento socialista riformista dal quadro ideologico del marxismo possa sfociare in una maggiore libertà ed efficacia dell'azione politica.

Sullo stesso numero della «Critica sociale» compare Le attività del bilancio ${ }^{17}$, un articolo di Rodolfo Mondolfo, cui la redazione aveva chiesto di formulare un commento sulle posizioni espresse da Rosselli. Il giudizio di Mondolfo non è liquidatorio; contiene anzi il riconoscimento di un'apprezzabile intraprendenza intellettuale. Ciò non toglie chel'intervento del filosofo marchigiano corrisponda all'intenzione di sottolineare il limite dell'analisi teorica di Rosselli, il fraintendimento del rapporto tra socialismo e marxismo, la semplicistica eliminazione di quest'ultimo come impedimento a una efficace azione socialista.

${ }^{15}$ Ivi, p. 91-92.

${ }^{16}$ Ivi, p. 92.

${ }^{17}$ R. Mondolfo, Le attività del bilancio, "Critica sociale», 1-15 novembre 1923, ora in Id., Umanismo di Marx. Studi filosofici 1908-1966, con introduzione di Norberto Bobbio, Einaudi, Torino 1975, pp. 228-233 (ed. orig. 1968). 
Secondo Mondolfo, ogni ideale deve essere sorretto da un impianto dottrinale adeguato. Senza validi criteri di interpretazione della realtà, si rischia di cadere in un atteggiamento in tanto utopistico, in quanto privo di una indicazione chiara del percorso da seguire. Il marxismo è la "bussola" che orienta l'azione socialista, "la coscienza storica del movimento proletario: coscienza critica, fatta guida e norma all'attività pratica; che nel materialismo storico ricerca la consapevolezza che la lotta di classe deve avere di se stessa, delle sue possibilità e del cammino progressivo delle sue conquiste $»^{18}$. Se per Rosselli la dottrina marxista irretisce le potenzialità del movimento socialista, in Mondolfo è assunta come indispensabile strumento per individuare modalità e tempi dell'azione storica,

[...] che ha bisogno di essere guidata e sorretta continuamente da una coscienza critica: quella esigenza di non proporsi se non problemi storicamente possibili, e fini raggiungibili, le cui condizioni, cioè, si trovino, se non già raggiunte, almeno nel processo del loro divenire, costituisce la norma fondamentale di ogni prassi storica; e basterebbe da sola a giustificare l'ispirazione marxistica del moderno movimento proletario ${ }^{19}$.

Tale esigenza dovrebbe essere maggiormente avvertita, e considerata anzi imprescindibile, proprio dai socialisti riformisti, poiché

[...] che cos'è il materialismo storico, che cos'è la concezione criticopratica, che cos'è la dipendenza sempre riaffermata dei problemi e dei fini dalle condizioni raggiunte o in processo di divenire, se non la teoria entro la quale il gradualismo trova il suo più organico e coerente inquadramento ${ }^{20}$

Rosselli replica al commento di Mondolfo, puntualizzando alcuni aspetti del suo pensiero in un nuovo intervento sulla «Critica sociale» dal titolo Aggiunte e chiose al "Bilancio marxista» ${ }^{21}$. La divergenza, ormai è chiaro, non riguarda tanto l'interpretazione della teoria marxista, quanto il rapporto di subordinazione della prassi socialista al marxismo. Preso atto della lunga attività revisionistica che ha fatto emergere la contraddittorietà, o almeno la mancanza di univocità, del modello marxista, Rosselli mette l'accento sugli assunti marxisti che non trovano riscontro empirico; né dal punto di vista dell'analisi economica (teoria del valore), né in relazione «al concreto processo del divenire socialista (catastrofismo)»»

${ }^{18}$ Ivi, p. 230.

${ }^{19}$ Ivi, p. 231.

${ }^{20}$ Ivi, p. 233.

${ }^{21}$ C. Rosselli, Aggiunte e chiose al «Bilancio marxista», «Critica sociale», 1-15 dicembre 1923, ora in Id., Opere (vol. 1), cit., pp. 96-106.

${ }^{22}$ Ivi, p. 99. 
Pertanto, non è più possibile sostenere la stretta dipendenza tra marxismo e "coscienza proletaria", fondandosi sulla previsione di una finale vittoria di classe. Materialismo storico e lotta di classe non sono, nell'interpretazione di Rosselli, elementi necessariamente determinanti l'azione socialista, sì che «il marxismo non può più esercitare nel partito - come partito - quella funzione di differenziazione, e soprattutto di bussola che esercitò nel passato $»^{23}$.

In effetti, l'andamento della polemica, e lo stesso giudizio conclusivo sul socialismo-marxismo, rispondono a una ragione essenziale di disaccordo rispetto al concetto di classe; se in Mondolfo il marxismo rappresenta la legittimazione teorica dell'ascesa del proletariato e la «coscienza storica» di una classe filosoficamente imputata di una missione storicouniversale, per Rosselli esso può offrire, e solo in alcune sue parti, un criterio di indagine e di orientamento critico. La sensibilità di economista non dottrinario e l'approccio empirico producono una visione aperta, mutevole, della composizione in classi della società, per cui «il graduale realizzarsi del moto socialista pone in luce la relatività e il valore solo teorico delle nette antitesi d'un tempo. Borghesia e proletariato non sono più (e dal lato economico non furono mai) due blocchi uniformi di forze» ${ }^{24}$. Alla contrapposizione cristallizzata in classi, Rosselli oppone dunque la convinzione secondo cui c'è più realismo nell'impegno di «lotta contro il regime, l'assetto economico - politico - giuridico che produce [...] tutti i mali della società contemporanea, piuttosto che nella lotta contro questo o quel gruppo sempre più difficilmente riconoscibile e perseguibile» ${ }^{25}$. $\mathrm{E}$ questa una tesi legata alla consapevolezza del discrimine introdotto dal fascismo. L'impasse scaturita dalla disgregazione dell'opposizione di classe potrebbe ancora trovare una soluzione qualora prevalesse in campo socialista la variabile unitiva antifascista. Il socialismo insomma dovrebbe tradursi in magnete di aggregazione funzionale a un impegno di libertà in chiave progressista, dando "più peso alle esigenze morali ed alle tendenze volontaristiche $»^{26}$.

Rosselli sembra scorgere i limiti di vaghezza idealistica della sua critica. Per dare maggior forza pragmatica alla sua proposta, finisce col concedere un apparente recupero delle indicazioni realistiche contenute nella visione marxista. Il marxismo può «concorrere a dare una utile coscienza critica dei limiti, delle possibilità dell'azione socialista», senza per ciò rappresentare «l'indispensabile chiave per la comprensione e, quindi, risoluzione dei grandiosi problemi concreti ed attuali $»^{27}$. Si tratta di un richiamo di ascendenza crociana che non pregiudica la liquidazione dell'identità tra

\footnotetext{
${ }^{23}$ Ibid.

${ }^{24}$ Ivi, p. 101.

${ }^{25}$ Ibid.

${ }^{26}$ Ivi, p. 103.

${ }^{27}$ Ivi, p. 104.
} 
dottrina e movimento. Nella cassetta degli attrezzi, c'è spazio anche per l'epistemologia marxista, al fine di analizzare di volta in volta i dati della realtà e quindi calibrare al meglio la portata dell'azione. La conclusione dell'analisi sul rapporto tra teoria e prassi del socialismo rimane in ogni caso la negazione dell'utilità di un partito marxista, "che codifichi cioè ed imponga [...] a tutti i suoi aderenti una determinata filosofia, una determinata visione della vita e delle lotte politiche ${ }^{28}$.

Il confronto si conclude con un ulteriore articolo di Mondolfo, anche questa volta chiamato in causa dalla «Critica sociale» per controbattere alle tesi di Rosselli. In Contributo ad un chiarimento di ide ${ }^{29}$, il filosofo marxista ribadisce l'impegno alla comprensione dello "spirito" di Marx, indispensabile guida del movimento proletario, con ciò negando che l'urgenza attivistica rivendicata da Rosselli venga frustrata da una dottrina contraddittoria e fondamentalmente deterministica ${ }^{30}$. Mondolfo si richiama alla sua opera principale, Sulle orme di Marx. Qui, come si è detto, attraverso il principio del rovesciamento della praxis aveva tentato di rivalutare il momento della volontà rispetto a quello della necessità, stabilendo tra i due un complesso rapporto dialettico. Così che l'azione dell'uomo non sarebbe subordinata, né tanto meno determinata dallo sviluppo delle condizioni economiche oggettive; queste ultime rappresenterebbero semplicemente un limite, da riconoscere e su cui intervenire, e, allo stesso tempo, agirebbero sull'uomo, modificandolo. Con riferimento alle famose Glosse su Feuerbach, Mondolfo attribuisce allo stesso Marx la definizione del concetto di praxis che si rovescia, che significherebbe «l'effetto che si trasforma in causa, il fatto storico che si tramuta in fattore di storia», così che

[...] il resultato dell'attività umana tende a diventar condizione e legge della propria creatrice, il prodotto vuol quasi dominare il produttore: ma, a sua volta, questo attivo, che rischia di esser mutato in passivo, reagisce e supera le condizioni che tendevano ad imporglisi: se non che viene, con l'opera sua medesima, a creare nuove condizioni, che tenderanno a dominarlo, e contro le quali l'attività successiva dovrà rovesciarsi ${ }^{31}$.

Questo concetto chiave consente di attribuire alla volontà dell'uomo un ruolo essenziale nello sviluppo del processo storico. Mondolfo sottolinea la differenza tra il materialismo storico e il determinismo economico:

${ }^{28}$ Ivi, p. 105.

${ }^{29}$ R. Mondolfo, Contributo ad un chiarimento di idee, «Critica sociale», 1-15 gennaio 1924, ora in Id., Umanismo di Marx, cit., pp. 234-241.

${ }^{30}$ Paolo Bagnoli ha visto proprio nel determinismo il tema cruciale di confronto tra Rosselli e Mondolfo. Cfr. P. Bagnoli, Carlo Rosselli. Il socialismo della libertà, cit.

${ }^{31}$ R. Mondolfo, Contributo ad un chiarimento di idee, cit., p. 95. 
[...] ossia la unità (che il primo sempre mantiene e il secondo rompe con l'ipostasi dell'economia) di ogni forma, aspetto e resultato dell'attività umana (siano le condizioni economiche, siano le politiche, giuridiche, religiose o quali altre si vogliano) con la sorgente dalla quale tutte derivano e in cui tutte si ripercuotono; con la energia rigeneratrice che tutte le produce e di tutte risente l'effetto nel modo di essere e di operare successivo: cioè l'uomo, che è il vero creatore della storia nella quale forma e trasforma, genera e condiziona se stesso e tutto l'indefinito svolgersi del proprio sviluppo, e dei risultati, prodotti ed effetti di questo, continuamente convertentesi in fattori, cause e condizioni-limiti ed ostacoli da un lato, e per ciò stesso d'altro lato impulsi e stimoli all'ulteriore progressivo svolgimento ${ }^{32}$.

La centralità dell'azione umana non viene dunque negata; semmai iscritta in un disegno dialettico in cui coscienza della realtà e fine di emancipazione convivono sotto l'egida di un pensiero organico. Mondolfo afferma che vi sarebbe «una visione attivistica e volontaristica [...] anche nel Capitale. Ma è sempre, qui come altrove, un volontarismo concreto, in rapporto con la realtà delle condizioni, dalle quali vengono gli stimoli all'azione, e sulle quali si deve operare ${ }^{33}$.

L'interpretazione di Mondolfo fornisce un esempio del rapporto tra revisionismo e riformismo. La palese divergenza tra gli obiettivi della prassi politica e le previsioni dottrinali viene superata attraverso l'adesione allo "spirito" del marxismo, collante ideologico dal quale non si vuole prescindere. Emerge così una formula che, mentre legittima il gradualismo, in nome del rovesciamento della praxis, non pregiudica l'orizzonte finalistico. La dottrina marxista non è, infatti, soltanto il faro costante dell'azione socialista nel suo continuo dispiegarsi, rappresenta altresì il fondamento teorico del fine universalistico di cui il proletariato è l'agente storico. È la prospettiva teleologica, che, anche in un marxismo gradualista e non determinista, è associata all'immagine dello scontro tra borghesia e proletariato come passaggio verso la liberazione "dell'umanità intiera da ogni divisione di classi» ${ }^{34}$. L'ideale etico, utopistico, diviene realizzazione storica attraverso il lume filosofico del marxismo che dà «al movimento proletario una coscienza storica di se stesso e dei suoi fini, e l'orientamento efficace della sua azione $»^{35}$. Dietro le formule che indulgono allo scolasticismo, rimane una preoccupazione non priva di fondamento, che giustifica l'insistenza sulla funzione indispensabile del marxismo come criterio di orientamento di un'azione socialista, esposta altrimenti al rischio di divenire dispersiva e inefficace. La preoccupazione riguarda

\footnotetext{
${ }^{32}$ Ivi, p. 218-19.

${ }^{33}$ Ivi, p. 237.

${ }^{34}$ Ivi, p. 238.

${ }^{35}$ Ivi, p. 239.
} 
le possibili conseguenze del disancoraggio da una ideologia forte, capace di generare identificazione collettiva, senso del fine, coesione morale, orgoglio di appartenenza. Semmai, si può attribuire a Mondolfo un errore di valutazione nel non prevedere la potenzialità egemonica del marx-leninismo, che nella versione del comunismo italiano scalzerà il connubio fragile e contraddittorio tra filosofia di Marx e riformismo umanitario di tradizione socialista.

Il contrasto tra Mondolfo e Rosselli concerne in definitiva tanto il problema, ovvero la possibile identità di un fine storico ultimo, quanto lo statuto filosofico e culturale da infondere al mezzo.

Il socialismo rosselliano, un socialismo verso cui tendere, eterodosso, intransigente sui valori ma "relativista" sulle formule della dogmatica, confligge con l'assoluto socialista che Mondolfo lega ai destini della filosofia marxista della storia.

\section{Il rapporto con il socialismo inglese}

Rosselli si era recato una prima volta in Inghilterra nell'autunno del 1922 per approfondire i suoi studi economici e politici senza trarne grande vantaggio. Significativa, invece, la permanenza a Londra nell'estate del $1923^{36}$. Se la cultura anglosassone rappresentava da sempre un modello di riferimento nel retroterra formativo di Rosselli ${ }^{37}$, è col viaggio del 1923 che egli viene a conoscenza diretta dell'ambiente socialista e dei suoi personaggi di spicco, cogliendo e valutando le differenti proposte provenienti dall'eterogenea famiglia laburista. Partecipa alla "quindicina" fabiana ${ }^{38}$, dove ha la possibilità di incontrare e ascoltare i coniugi Webb; frequen-

${ }^{36}$ In una lettera del 30 agosto 1923 , commentando la sua nuova esperienza inglese, Rosselli scrive: «il contrario dell'anno scorso, il viaggio attuale. Poche cose morte, e molte cose vive. E qualche esperienza in più» (Epistolario familiare, cit., p. 168).

${ }^{37} \mathrm{Si}$ vedano i capitoli II e III del testo di S. Mastellone, Carlo Rosselli e «la rivoluzione liberale del socialismo», Olschki, Firenze 1999, pp. 21-55, il quale traccia un lungo filo conduttore che lega Rosselli alla cultura politica inglese, dall'adolescenza alla maturità. Si fa riferimento all'opera di autori di cultura liberale, definibili come antesignani del liberalismo sociale, come J.S. Mill, L.T. Hobhouse o J.A. Hobson, e a quella di alcuni rappresentanti nobili del socialismo inglese come i coniugi Webb, G.D.H. Cole e J. Ramsay MacDonald. Per un'analisi del liberalismo socialista nella tradizione inglese si veda $\mathrm{N}$. Urbinati, Il liberalismo socialista nella tradizione inglese, in M. Bovero, V. Mura, F. Sbarberi, (a cura di), I dilemmi del liberalsocialismo, cit., pp. 211-236. La Urbinati ha indicato più specificamente nei Principles of Political Economy di J.S. Mill l'opera anticipatrice sull'avvicinamento tra liberalismo e socialismo.

${ }^{38}$ Una delle riunioni della Fabian Summer School che si tengono presso la St. Edmund's School (Surrey). Questa partecipazione è narrata nella lettera di Carlo da Londra del 28 luglio 1923, in Epistolario familiare, cit., p. 160. 
ta la London School of Economics, si reca al congresso annuale delle Trade Unions ${ }^{39}$. Attraverso Richard H. Tawney, conosce inoltre George D.H. Cole, che definisce come «uno degli intellettuali più suggestivi nel movimento cooperativo gildista inglese ${ }^{40}$.

Le conoscenze acquisite durante questa esperienza aprono una nuova fase di riflessione per Rosselli. L'Inghilterra, vista come esempio di civiltà moderna e liberale, diviene fonte di riferimenti concreti per la definizione di una proposta politica e per la ricerca di un progetto strutturale di cambiamento economico. Del socialismo inglese, egli apprezza in particolare il metodo tipicamente pragmatico, empiristico, così poco permeato dall'ideologia marxista. Ė un apprezzamento dettato da una sensibilità culturale che è maturata negli anni e che trova nel modello della dialettica politica e sociale britannica un sicuro riscontro. Il giudizio sul laburismo inglese indica un implicito confronto con l'esperienza del socialismo italiano: le tribolazioni del secondo condizionano e acuiscono l'entusiasmo per il primo ${ }^{41}$.

Il $1^{\circ}$ febbraio 1924 viene pubblicato sulla «Libertà» l'articolo Il partito del lavoro in Inghilterra ${ }^{42}$. Rosselli parte da una ricostruzione storica del movimento laburista per individuare gli elementi che ne hanno determinato la forza. Sottolinea innanzitutto l'origine e l'impronta sindacalistica del socialismo inglese. Solo dopo un lungo processo di maturazione e consolidamento, le istanze economiche del tradeunionismo confluiscono in una forma-partito ${ }^{43}$, diversa dalla forma dei partiti socialisti di tipo continentale, in quanto «federazione di gruppi economici (sindacati, coo-

${ }^{39}$ Cfr. le lettere spedite alla madre dal 28 luglio al 2 settembre 1923, in Epistolario Familiare, cit., pp. 158-170.

${ }^{40}$ Rosselli fa riferimento a un «appuntamento per il 26 agosto! al tè!» (lettera del 28 luglio 1923, ivi, p. 160).

${ }^{41}$ Rosselli non è l'unico a guardare con interesse all'Inghilterra. Altri giovani, come Nino Levi, Alessandro Schiavi, Enrico Sereni e Pietro Sraffa (nella lettera alla madre del 15 dicembre 1923 Carlo fa riferimento ad una "conversazione sull'Inghilterra alla Università Proletaria che terrà Nino Levi» e parla anche della nuova conoscenza con Sraffa), seguono le vicende del socialismo d'oltremanica, alla ricerca di spunti innovatori. Il $1^{\circ}$ gennaio 1924 esce il primo numero del quindicinale "Libertà». La nascita di questa rivista è il risultato della pressione della nuova generazione socialista per ottenere un luogo di dibattito, cui viene incontro la vecchia classe dirigente del Partito Socialista Unitario. Cfr. S. Mastellone, Carlo Rosselli e «la rivoluzione liberale del socialismo», cit., pp. 36-37.

${ }^{42}$ Rosselli dà notizia dell'imminente pubblicazione dell'articolo nella lettera alla madre del 23 gennaio 1924, in Epistolario familiare, cit., p. 193.

${ }^{43} \mathrm{Il}$ sindacalismo inglese crebbe tutelando gli interessi della classe lavoratrice patteggiando "ora coi conservatori, ora coi liberali», fin quando non fu necessario «entrare risolutamente nel campo politico con uomini e partito propri» (C. Rosselli, Il Partito del Lavoro in Inghilterra, ora ripubblicato in appendice al volume di S. Mastellone, Carlo Rosselli e «la rivoluzione liberale del socialismo», cit., pp. 142-151. La citazione è tratta dalla p. 145). 
perative) e di gruppi politici» ${ }^{44}$. Per Rosselli ciò permetterebbe al Labour Party di essere l'espressione politica del movimento operaio, portatore di interessi concreti e non mosso da parti ideologici, «mentre sul continente è l'idea, l'ideologia marxista che attraverso i partiti crea l'organizzazione economica tentando [...] di subordinarla ai suoi fini» ${ }^{45}$.

La rappresentazione è schematica, ma vi è dietro l'ennesimo tentativo di indicare i limiti di un ideologismo pagato a caro prezzo dal socialismo italiano. Rosselli vede nel laburismo una forza duttile, ispirata da una cultura capace di tenere insieme anime diverse grazie a un «largo spirito liberale, una così ampia autonomia, una così larga libertà di movimento $\mathrm{e}$ di critica, che la necessità di una scissione almeno nel periodo negativo di opposizione fu sempre evitata ${ }^{46}$. È questo un modo indiretto per ribadire il giudizio negativo verso l'asfittica fedeltà a un sistema dottrinario che limita l'efficacia e la libertà dell'azione politica. Secondo Rosselli in Inghilterra «il marxismo, al pari di ogni altra ideologia aprioristica, non ha mai attecchito, se si tolgono sparuti gruppi di intellettuali», e il dito viene puntato contro un'interpretazione del materialismo storico «inteso e frainteso generalmente come basso e volgare determinismo economico», che in Inghilterra verrebbe "nettamente respinto ${ }^{47}$. Ciò che potrebbe apparire come indifferentismo ideologico è, nella visione rosselliana, volontarismo idealistico frammisto a pragmatismo: entrambi sinergicamente a fondamento della tradizione operaia britannica, in cui la lotta di classe è lotta «contro il regime capitalistico che produce i mali e le ingiustizie a tutti note» e non «lotta contro questa o quella classe. Perciò si cercano appoggi in tutte le classi, in tutte le categorie della popolazione, qualunque sia la loro condizione, purché concordi grosso modo nei fini e nei metodi» ${ }^{48}$.

${ }^{44}$ Ivi, p. 142.

${ }^{45}$ Ibid.

${ }^{46}$ Ivi, p. 146. Sulle considerazioni riguardanti l'organizzazione del partito laburista, Mastellone ha affermato che «nel quadro politico italiano questa valutazione positiva della organizzazione interna del partito laburista inglese è abbastanza personale. La socialdemocrazia tedesca, caratterizzata da una sostanziale adesione al marxismo, era il modello di riferimento tanto ideologico quanto strutturale del socialismo italiano. Il partito socialdemocratico tedesco fin dal congresso di Erfurt, tenutosi nell'ottobre 1897, aveva [...] adottato non solo il pensiero di Marx, ma un ordinamento interno rigido e autoritario» (S. Mastellone, Il Partito politico nel Socialismo liberale di Carlo Rosselli, «Il pensiero politico», Anno XXXI, 1998, N. 1, p. 113). Cfr. anche A. De Sanctis, Il problema del partito in Rosselli e Hobhouse, «Il Pensiero Politico», Anno XXXIII, 2000, N. 1, pp. 38-63.

${ }^{47}$ C. Rosselli, Il Partito del Lavoro in Inghilterra, cit., p. 147.

${ }^{48} \mathrm{Ibid}$. In quest'ottica Sbarberi ha sostenuto che Rosselli non può sentirsi attratto dalle "categorie analitiche del Capitale, bensì, pragmaticamente, dall'esperienza cooperativistica e mutualistica di un movimento politico come il Labour Party, aperto ai contributi ideologici più differenziati e capace di affrontare i problemi della giustizia sociale senza remore di principio» (F. Sbarberi, L'utopia della libertà eguale, cit., p. 63). 
In un momento storico in cui la prospettiva classista è oggetto di ripensamenti, revisioni o rivalutazioni, mentre un personaggio come Gobetti guarda con slancio rinnovato a L'ora di Marx, Rosselli lega sempre più il bilancio della tradizione socialista italiana alla scissione dai residui marxiani ${ }^{49}$.

L'apprezzamento per il laburismo trova una giustificazione supplementare nel successo elettorale che aveva condotto alla formazione del governo $\mathrm{McDonald}^{50}$, per Rosselli un segno inequivocabile della possibilità di perseguimento del socialismo attraverso il rispetto delle istituzioni politiche liberali: anzi di più, l'indicazione della presa del potere come premessa non per cambiare le "regole del gioco", bensì per dare maggior forza (ben oltre l'azione dei movimenti economici) al progetto della trasformazione sociale. Così «grande merito del Labour Party fu quello di aver costantemente mirato, sin dall'inizio della sua azione contenuta nei limiti costituzionali, alla conquista del potere», e aver compreso che «non era il caso di insistere troppo sui limiti classistici del movimento» ${ }^{51}$. Emerge, dunque, anche il tema del superamento dei confini di schieramento secondo basi rigidamente di classe, sì da conquistare «larghe simpatie nei ceti borghesi e molti elementi politici liberali» $»^{52}$.

Prendendo spunto dalle vicende inglesi, Rosselli crede di poter ribadire la sua visione del socialismo come erede del liberalismo e ritiene che «la storica funzione liberale intesa nel senso più largo della parola, rinvigorita dall'apporto grandioso del riformismo socialista del mondo operaio, si trasferirà dal classico Partito Liberale al nuovo e battagliero Partito La-

${ }^{49}$ Revelli, rispetto al confronto Rosselli-Gobetti, sottolinea che «nel tentativo di riavvicinare i due termini - socialismo e liberalismo -, l'uno, Rosselli, partendo da premesse socialiste si allontanasse progressivamente da Marx mentre l'altro, Gobetti, partendo da premesse liberali, vi si avvicinasse progressivamente» (M. Revelli, Gobetti "liberal-comunista"?, cit., p. 77). A proposito dell'articolo L'ora di Marx, pubblicato sulla "Libertà» il $1^{\circ}$ aprile 1924, Mastellone ha ricordato come «l'intervento di Gobetti non migliorò i rapporti con Carlo Rosselli» (S. Mastellone, Carlo Rosselli e «la rivoluzione liberale del socialismo», cit., p. 41).

${ }^{50}$ Lo stesso Claudio Treves aveva salutato con favore la vittoria laburista in un articolo intitolato Dopo le elezioni britanniche, "Critica Sociale», 16-31 dicembre 1923.

${ }^{51}$ C. Rosselli, Il Partito del Lavoro in Inghilterra, cit., p. 148.

${ }^{52} \mathrm{Ibid}$. Queste argomentazioni vanno evidentemente lette con riferimento alla situazione italiana. La frustrazione per l'incapacità dei socialisti di contrastare la deriva fascista, uscendo dalla logica aventiniana, pesa su certe posizioni di Rosselli. Ed è pure evidente che la proposta rosselliana non è immediatamente applicabile al contesto storico, sociale e ideologico di quegli anni. Come ha sostenuto Tranfaglia, certe posizioni di Rosselli più che esprimere «un meditato giudizio politico» inseguivano il sogno di «un socialismo moderato e gradualista, non classista, fedele ai princìpi della democrazia liberale, capace di ottenere i consensi del proletariato e della borghesia avanzata: tale sogno rappresentava per lui un'esigenza ideologica di fondo, a cui tenterà di dare un'espressione globale nel '30 con Socialism libéral») (N. Tranfaglia, Carlo Rosselli dall'interventismo a «Giustizia e Libertà», cit., p. 190). 
bourista $»^{53}$. È evidente che la posizione di Rosselli nel dibattito sul Che fare?, interno alla sinistra italiana, è assai critica, ma saldamente rivolta a un orizzonte socialista ${ }^{54}$. A giustificare una scelta di campo tanto netta, non è esclusivamente il richiamo del dovere morale, ma una convinzione ideologica, che può apparire confusa e incompleta in quanto aperta e disorganica.

Se da un lato Rosselli si getta nella lotta politica, fermamente convinto dell'importanza del ruolo di un partito riformista, dall'altro non perde di vista la ricerca di una formula capace di determinare cambiamenti di "sistema”. Il punto di riferimento è sempre l'Inghilterra. È un fatto che nel movimento tradeunionista e laburista convivano esperienze diverse, con progetti di realizzazione del socialismo anche contrapposti. Attraverso un approfondimento delle sue ricerche sul sindacalismo, Rosselli tenta appunto di analizzare e confrontare quelle diverse esperienze, consolidando una visione antistatalistica e autonomistica della emancipazione del lavoro.

Nel marzo del 1924, «La Rivoluzione Liberale» dedica un numero unico all'Inghilterra, al quale Rosselli contribuisce con l'articolo Il movimento operai $^{55}$. La considerazione iniziale riguarda il limite dell'azione sindacale, intesa come attività rivendicativa, che può incidere positivamente sul piano del miglioramento delle condizioni salariali, ma che risulta inadeguata alla trasformazione della struttura economica capitalistica. In realtà, secondo Rosselli, il movimento sindacale è «del tutto impotente a mutare stabilmente la quota relativa a remunerazione del lavoro nei confronti della quota relativa a remunerazione dei possessori di capitale $\aleph^{56}$. Si impone soprattutto la ricerca di strategie che determinino una reale democratizzazione dei rapporti economici. Proprio perciò Rosselli prende in considerazione l'esperienza inglese e mette a confronto due prospettive tendenzialmente opposte: quella statalistica e collettivistica dei Webb e quella gildista di Cole ${ }^{57}$.

${ }^{53}$ C. Rosselli, Il Partito del Lavoro in Inghilterra, cit., p. 151.

${ }^{54}$ Il 3 ottobre 1924 Carlo comunica alla madre il suo ingresso nel P.S.U., scrivendo: «avrai forse letto nella "Giustizia" la nostra dichiarazione di adesione al Partito Socialista Unitario. Come vedi con Limentani, Salvemini, Jahier, l'economista Gino Luzzato ed altri sono proprio in buona compagnia» (Epistolario familiare, cit., pp. 221-222). E in una lettera indirizzata a Gobetti, Rosselli afferma come «sia venuta l'ora per tutti di assumere il proprio posto di battaglia in seno ai partiti» (citato in N. Tranfaglia, Carlo Rosselli dall'interventismo a «Giustizia e Libertà», cit., p. 178).

${ }^{55}$ C. Rosselli, Il movimento operaio, «La Rivoluzione Liberale», 25 marzo 1924, ora in Id., Opere (vol.1), cit., pp. 65-76. Nello stesso numero, intitolato Che cos'è l'Inghilterra, compaiono articoli di Annibale Coduri, Mario Borsa, Guido De Ruggiero, F. Paolo Giordani, Nino Levi, Antonio Crespi, Giovanni Ansaldo («Rivoluzione liberale», Anno III, n. 13-14). Carlo fa riferimento all'«articolo per "Rivoluzione Liberale" sul gildismo» in una lettera senza data spedita da Milano (Epistolario familiare, cit., p. 207).

${ }^{56}$ C. Rosselli, Il movimento operaio, cit., p. 66.

${ }^{57}$ Cfr. D. Marucco, Fabianesimo, glildismo, forme di democrazia industriale, Milano, FrancoAngeli 1986 e C. Palazzolo, Dal fabianesimo al neofabianesimo: iti- 
Il progetto fabiano è incardinato nella visione filosofica del positivismo e sottende un'idea evoluzionistica della società ${ }^{58}$. L'obiettivo coincide con l'adozione di criteri di efficienza che consentano il miglioramento graduale e costante delle condizioni economiche. Il socialismo è identificato come meta per la presunta superiorità produttiva e nell'allocare risorse, in virtù della trasformazione della proprietà e non perchè modifica l'organizzazione del lavoro e la sua costituzione gerarchica ${ }^{59}$. I Webb immaginano la fondazione di una democrazia dei consumatori, che ruoti intorno a un sistema di cooperative di consumo, imprese pubbliche e municipalizzate. Nel quadro di un socialismo che si sviluppa più secondo ragioni di opportunità che di valore, forme di proprietà privata sono inoltre destinate a sopravvivere per essere progressivamente assorbite da enti pubblici. L'organizzazione economica dovrebbe dunque essere sottoposta a successive riforme che trasferiscono il governo della produzione dal privato al pubblico, per "fisiologiche" ragioni di prestazioni più soddisfacenti.

L'amministrazione dell'industria, secondo i Webb, consiste di tre funzioni principali: innanzitutto «la determinazione di ciò che dovrà prodursi», in secondo luogo «il criterio del modo in cui la produzione dovrà essere compiuta, l'adozione della materia prima, la scelta dei metodi industriali e la scelta degli agenti umani», ed infine «la questione completamente diversa delle condizioni in cui questi agenti umani dovranno essere impiegati», condizioni ambientali, igieniche e salariali ${ }^{60}$. I primi due elementi del governo d'impresa dovrebbero essere sottratti al contributo della classe lavoratrice e gestiti secondo parametri di produttività finalizzati alla massima soddisfazione dei consumatori. Rivelando ascendenze saintsimoniane, i Webb vorrebbero affidare la direzione dell'economia ai nuovi "sacerdoti" della competenza. Il nucleo dei tecnocrati può essere formato, secondo i casi, da «un corpo di pubblici funzionari stipendiati intenti a soddisfare associazioni di consumatori (come nel Movimento cooperativista britannico) o associazioni di cittadini (il Municipio o

nerario di storia della cultura socialista britannica, Giappichelli, Torino 1999. Sulla conoscenza della letteratura socialista inglese da parte di Rosselli vi sono giudizi storici discordanti. Per Mastellone questa sarebbe già maturata nel periodo della prima tesi di laurea; Tranfaglia ha sottolineato invece come solo dopo il 1923 emerga una chiara consapevolezza della diversità di progetti espressi dal variegato universo del socialismo inglese. Cfr. S. Mastellone, Carlo Rosselli e «la rivoluzione liberale del socialismo», cit., pp. 21-26 e N. Tranfaglia, Carlo Rosselli dall'interventismo a «Giustizia e Libertà», cit., pp. 161-164.

${ }^{58}$ Per Beilharz, questa concezione considera il socialismo una «tendenza evolutiva» di cui il fabianesimo rappresenta la «culminazione formale» (P. Beilharz, Labour's Utopias, Routledge, London, New York, 1992, p. 52).

${ }^{59}$ Sul mantenimento di un articolato ordine gerarchico nel progetto di costituzione fabiana, cfr. L. Rockow, Contemporary Political Thought in England, George Allen \& Unwin LTD, London 1930, p. 111 (ed. orig. 1925).

${ }^{60} \mathrm{~S}$. Webb, B. Webb, La democrazia industriale, Unione Tipografico-Editrice Torinese, Torino 1912, p. 747 (ed. orig. 1897). 
lo Stato)», o anche, in una prospettiva intermedia di economia mista, da "capitalisti che realizzano i profitti, fortissimamente interessati a guadagnarsi la clientela ${ }^{61}$.

La parte riconosciuta ai sindacati dei lavoratori riguarda quindi solo l'ultima funzione, quella rivendicativa ${ }^{62}$. Nella cornice ideale di un socialismo destinato ad affermarsi democraticamente, la presenza del sindacato supporta l'azione politica del partito, allo scopo di realizzare il programma del "Minimum Nazionale", ossia «l'imposizione sistematica, per mezzo di un Codice del Lavoro minutamente elaborato, di una quota determinata di istruzione, di misure igieniche, di periodi di riposo e di salarii per ogni grado di lavoratori in qualsiasi industria» ${ }^{63}$. Il funzionamento del sistema sarebbe garantito dallo Stato, secondo una concezione olistica della società, per cui «nessun gruppo ha un'autorità incondizionata nemmeno nella propria sfera. Lo Stato è socio in ogni intrapresa ${ }^{64}$. E poiché, secondo i Webb, «la democrazia si muove nel senso di sostituire tanto il piccolo imprenditore che lavora pel proprio profitto, quanto il grande Sindacato Industriale, col funzionario stipendiato della Società cooperativa, del Municipio, e dell'Azienda governativa» ${ }^{65}$, a delinearsi è una forma di socialismo collettivistico, con lo Stato nella veste di istituzione votata alla sintesi di istanze provenienti da diversi soggetti, capace di produrre un superamento della lotta di classe e di governare l'economia attraverso manager pubblici ${ }^{66} . \mathrm{Si}$ dovrebbe così pervenire $\mathrm{a}$ un incremento $\mathrm{e}$ a una più equa redistribuzione della ricchezza e dunque all'emancipazione dal bisogno dell'intera comunità, presupposto dell'elevazione spirituale degli uomini.

Rosselli non condivide la concezione che ispira queste proposte. Attento ai temi del controllo operaio e dell'autogoverno nell' industria, commenta ironicamente le tesi dei Webb, sostenendo che

[...] con questo roseo epicureismo il sogno cooperativo è compiuto. Lo sforzo di emancipazione operaia è spacciato. La servitù del mondo economico non scompare, si trasforma; servi dell'umanità, non più del

${ }^{61}$ Ibid.

${ }^{62}$ Escludendo l'ipotesi che le «unioni potessero diventare corpi politici» (G. Foote, The Labour Party's Political Thought, Croom Helm, London 1985, p. 31).

${ }^{63}$ S. Webb, B. Webb, La democrazia industriale, cit., p. 746.

${ }^{64}$ Poiché nell'interesse «della comunità considerata come un tutto a nessuna delle interminabili serie di decisioni può essere consentito di andare contro il consenso dell'opinione degli uomini sperimentati che rappresentano i consumatori da un lato, i produttori dall'altro, e la nazione che domina sovrana sugli uni e sugli altri» (ivi, p. 751).

${ }^{65}$ Ivi, p. 752.

${ }^{66}$ Secondo un principio di razionalismo tecnocratico che dovrebbe eliminare il rischio di arbitrarietà nel governo dell'industria e della società, e in cui, quindi, «la scienza è destinata a rimpiazzare il capriccio, la fantasia o l'amor proprio» (L. Rockow, Contemporary Political Thought, cit., p. 112). 
privato sfruttatore. E la questione sociale è risolta, la pace assicurata, il socialismo realizzato ${ }^{67}$.

Più congeniale alla sua sensibilità è l'approccio di Cole, che insiste sull'intima connessione tra il valore dell'autogoverno e il perfezionamento morale della classe lavoratrice e individua i pericoli che possono derivare dal collettivismo accentratore (nemico giurato del gildismo, scuola di pensiero che, come si è accennato, trae ispirazione culturale dall'esperienza delle corporazioni medievali). Facendo battere l'accento sul rischio che venga trascurata la componente etica del socialismo, Rosselli insiste sulla convinzione che l'operaio "cosa, numero, materia grigia estranea alla vita della fabbrica moderna deve riacquistare in seno alla fabbrica [...] tutta la sua personalità. Il problema operaio è problema di coscienza, di dignità, di libertà» ${ }^{68}$. La felicità non può costituire un dono dello Stato. Il principio di autonomia deve tenere conto delle prerogative dell'individuo. La democrazia economica non può ridursi a mera giustizia redistributiva, ma deve promuovere il coinvolgimento attivo nel controllo dell'industria ${ }^{69}$, poiché il suffragio universale «è più una forza negativa [...] Finché nella organizzazione economica domina l'autocrazia, la casta, la divisione in classi, non si può parlare di vera democrazia» ${ }^{70}$.

Le considerazioni di Rosselli trovano riscontro nel socialismo di Cole, animato dalla spinta verso una rivoluzione morale. La democrazia gildista non è sinonimo di meccanicismo egualitario, ma partecipazione sociale, responsabilizzazione diretta del lavoratore, in quanto «salari più alti non renderanno meno monotona o automatica la vita del lavoratore, soggetto al controllo di un burocrate e separato da ogni libertà e responsabilità $\rangle^{71}$. In Cole è presente addirittura un'aspra critica del "wage-system", accusato di determinare la dissociazione tra lavoro e lavoratore e di produrre un fenomeno di alienante passività rispetto alle scelte del processo produttivo. Da qui l'impegno a contrastare la logica capitalistica, che offre alimento a un sistema «in cui il lavoro viene comprato e venduto nel mercato del lavoro come un articolo di commercio ${ }^{72}$. Sotto tale profilo, il controllo operaio è concepito come un mezzo in grado di stimolare la consapevolezza necessaria per conseguire il fine, vale a dire l'abolizione del capita-

${ }^{67}$ C. Rosselli, Il movimento operaio, cit., p. 70.

${ }^{68}$ Ivi, p. 71.

${ }^{69}$ Non c'è dubbio che «alla via legislativa, mediante l'imposta, l'espropriazione e l'intervento dello Stato, egli preferiva la via sindacale-cooperativa», come ha sostenuto M. Degl'Innocenti, Carlo Rosselli e il movimento sindacale: dalla tesi di laurea a Socialismo liberale, cit., p. 64.

${ }^{70}$ C. Rosselli, Il movimento operaio, cit., p. 73.

${ }^{71}$ G.D.H. Cole, Self-government in Industry, G. Bell and sons, LTD., London 1918, p. 53 (ed. orig. 1917).

${ }^{72}$ Ivi, p. 153. Sul sistema dei salari che «riduce gli uomini alla condizione di una macchina» cfr. G. Foote, The Labour Party's Political Thought, cit., p. 108. 
lismo non solo come modello economico ma anche come paradigma di valori. La radicalità dello scopo non esclude la gradualità degli interventi necessari a perseguirlo. Per Cole, il «controllo operaio» rappresenta «un primo passo verso una misura più completa di autogoverno industriale $»^{73}$, che dovrà infine condurre al completo «rovesciamento e sostituzione del capitalismo ${ }^{74}$, senza per questo dover ricorrere ad atti eversivi.

La lezione di Cole colpisce Rosselli per la venatura libertaria che la attraversa, oltre e forse più che per la strategia d'azione politica rivolta alla trasformazione integrale dei rapporti economici. L'autogoverno è il simbolo della riappropriazione del lavoro da parte del lavoratore e rinvia alla realizzazione individuale attraverso la condivisione di processi decisionali collettivi. L'autodeterminazione del lavoro coincide così con una prospettiva di socialismo umanistico, che punta all'innalzamento etico del soggetto attraverso un processo pandemocratico.

Cole in effetti individua un parallelismo tra il percorso del principio democratico in politica e la maturazione degli istituti di democrazia industriale. Le Trade Unions seguirebbero lo stesso andamento evolutivo dei Parlamenti, per cui

[...] in questo stadio, la loro influenza è ancora ridotta, poiché non hanno potere diretto in materia di legislazione industriale e controllo diretto sul governo industriale. Solo con la concessione di questo potere diretto e concreto finirà il periodo restrittivo e la democrazia diverrà il principio regolativo dell'organizzazione industriale ${ }^{75}$.

Il protagonista della democrazia è comunque l'individuo autonomo e cosciente, che si associa insieme ad altri individui e partecipa, senza delegare passivamente, poiché «la più completa democrazia di fatto è solo il logico sviluppo del principio del consenso, esteso attraverso l'applicazione della reale volontà umana - che è volontà di autonomia» ${ }^{76}$.

In coerente consequenzialità con la filosofia del governo dal basso, è presente in Cole una critica netta nei confronti delle tesi statalistiche, che trova terreno fertile nelle inclinazioni di Rosselli. Il pericolo è incarnato da un «onnicompetente, onnivoro, onnisciente, onnipresente Stato sovrano» ${ }^{77}$, sostenuto da un apparato burocratico che mortifica l'iniziativa individuale e impedisce l'effettiva emancipazione del lavoro con la costituzione di un

${ }^{73}$ G.D.H. Cole, Self-government in Industry, cit., p. 64.

${ }^{74}$ Ivi, p. 174.

${ }^{75}$ Ivi, p. 67.

${ }^{76}$ G.D.H. Cole, Social Theory, Methuen \& Co. LTD., London 1921, p. 92. In ossequio al principio rousseauviano per cui «nessun uomo può rappresentare un altro, la volontà di nessun uomo può essere rappresentata da un'altra» (L. Rockow, Contemporary Thought in England, cit., p. 153).

${ }^{77}$ G.D.H. Cole, Social Theory, cit., p. 11. 
opprimente capitalismo di Stato. In Self-government in Industry Cole si riferisce esplicitamente ai Webb, e alla loro "panacea dell'intervento statale $»^{78}$. Questa, in sostanza, si ridurrebbe a una formula che combina burocrazia amministrativa e autarchia industriale. Sono condotte a estrema contraddizione polemica due concezioni filosofiche diverse: l'organicismo positivistico del socialismo di Stato e il volontarismo autonomistico coincidente con il libero associarsi dei produttori. Questa contrapposizione trova una riduzione sinottica quando Cole afferma che la differenza «tra una gilda e un ministero, per quanto efficiente, è appunto questa: il secondo è un governo dall'alto e dall'esterno, la prima dal basso e dall'interno, autogoverno ${ }^{79}$. Ė evidente in Cole una visione pluralistica della società e delle istituzioni, che ha come premessa filosofica una concezione dell'uomo a più dimensioni; un uomo che sfugge alla riduzione olistica e che esalta la propria individualità in più sfere d'appartenenza ${ }^{80}$.

In effetti, quando afferma che con il «cadere del privilegio economico e col libero riorganizzarsi della produzione per opera di gruppi autonomi federali di produttori, avremo non più uno, ma due, ma più Stati», in quanto qualunque "associazione sostanzialmente è Stato» ${ }^{81}$, Rosselli fa riferimento esplicito alla teoria sociale di Cole. L'idea limite è quella di un soggetto che agisce in comunità attraverso appartenenze molteplici, in un tessuto associativo plurale che contemperi un ventaglio variegato di interessi specifici. Lo Stato sarebbe pertanto ridotto all'identità di governo, non più sintesi superiore delle diverse istanze sociali ${ }^{82}$, ma strumento di organizzazione e gestione politico-amministrativa. Cole distingue in modo netto Stato e società:

[...] quando devo fare riferimento al meccanismo organizzato del governo, nazionale e locale, parlerò di "Stato". Quando, invece, mi devo riferire all'insieme complesso delle istituzioni che consentono il funzionamento della comunità, parlerò di "Società"

È nella comunità-società (interessante notare la confusione sincretica dei due termini, a dimostrazione di un tentativo di sciogliere la tensione tra autonomia e appartenenza) che il singolo incontra le opportunità di impegnarsi attivamente in una o più associazioni con vocazione economica e sociale, mentre l'identificazione con l'ente Stato ha natura passiva. Il socialismo

${ }^{78}$ G.D.H. Cole, Self-government in Industry, cit., p. 60.

${ }^{79}$ Ivi, p. 297. Nella convinzione, come ha specificato P. Beilharz, che «semplicità e prossimità sono preferibili a complessità e ordine» (Labour's Utopia, cit., p. 76).

${ }^{80}$ L'obbligazione politica dell'individuo è così plurale e non «subordinata alla fedeltà dovuta allo Stato» (L. Rockow, Contemporary Political Thought in England, cit., p. 157).

${ }^{81}$ C. Rosselli, Il movimento operaio, cit., p. 73.

${ }^{82}$ Lo Stato non è riconosciuto come l'ente che rappresenta legittimamente la volontà generale (cfr. P. Beilharz, Labour's Utopia, cit., p. 78).

${ }^{83}$ G.D.H. Cole, Self-government in Industry, cit., p. 72. 
gildista dovrebbe quindi realizzarsi mediante nuclei di liberi produttori che si associano in cooperative capaci di attuare un coordinamento delle rispettive attività. Tutto ciò, anche per Rosselli sulle orme di Cole, nel quadro di un superamento della lotta di classe, per via incruenta e democratica ${ }^{84}$.

Tanto in Cole quanto in Rosselli, l'insistenza sull'autogoverno del lavoro deriva non solo da motivazioni di ordine morale, ma anche dalla consapevolezza dei limiti dell'azione sindacale ottocentesca, efficace nel migliorare le condizioni materiali di vita dei lavoratori, ma insufficiente a modificare il sistema capitalistico nella sua organizzazione e struttura. In Self-government in Industry, Cole solleva il problema della crisi dell'azione unionista incentrata sul principio rivendicativo di contrasto e contenimento del potere capitalistico, e prevede

[...] solo due possibili vie d'uscita. Una è quella folle dell'annientamento del sindacato, abbandonando le redini ai datori di lavoro, l'altra è la trasformazione del potere negativo di controllo, finora esercitato dai sindacati, in un potere positivo, mettendo nelle loro mani il potere esecutivo e legislativo ${ }^{85}$.

Il percorso analitico di Rosselli prosegue e si sviluppa in una serie di articoli pubblicati su «La Riforma sociale», nel periodo 1924-26, partendo dalla considerazione del sindacato in chiave antiliberista all'interno del sistema capitalistico. Gli interventi sulla rivista fondata da Nitti e che era stata punto di riferimento del liberalismo "anglofilo" aperto alle questioni sociali ${ }^{86}$, corrispondono a una ideale continuazione della polemica con Einaudi.

Rosselli attribuisce alla lega operaia la dignità di impresa economi$\mathrm{ca}$, che sorge «allo scopo di alienare sul mercato, col massimo provento possibile, la forza-lavoro detenuta singolarmente dai suoi membri» ${ }^{87}$. E sottolinea parimenti come la naturale vocazione al compattamento della rappresentanza del fronte del lavoro non sia un elemento di distorsione illiberale, bensì un fattore di equilibrio dei rapporti economici, che fa da contraltare alla forza crescente e coalizzata degli imprenditori ${ }^{88}$. Con ragionamento empiristico, Rosselli rimarca l'utilità collettiva conseguen-

${ }^{84}$ Cfr. C. Rosselli, Il movimento operaio, cit., pp. 72-73, e G. D. H. Cole, Self-government in Industry, cit., pp. 82-83.

${ }^{85}$ G.D.H. Cole, Self-government in Industry, cit., pp. 61-62.

${ }^{86}$ Cfr. C. Malandrino (a cura di), Una rivista all'avanguardia. La «Riforma sociale» 1894-1935. Politica, società, istituzioni, economia, statistica, Olschki, Firenze 2000, presentazione di G.M. Bravo, e G. Bianchi, Come cambia una rivista. La «Riforma sociale» di Luigi Einaudi 1900-1918, Giappichelli, Torino 2007.

${ }^{87}$ C. Rosselli, Scienza economica e leghe operaie, «La Riforma sociale», maggiogiugno 1924, ora in Id., Opere (vol. 1), cit., pp.197-198.

${ }^{88}$ Nelle sue valutazioni, Rosselli attinge alle analisi economiche condotte dall'inizio del secolo agli anni venti da Attilio Cabiati. Cfr. G. Becchio, Attilio Cabiati tra socialismo cooperativo e marginalismo, in M.E.L. Guidi, L. Michelini (a cura di), Marginalismo e socialismo nell'Italia liberale, cit., pp. 137-150. 
te al confronto "duopolistico", che tende ad «avvicinarci alle ipotetiche condizioni perché si verifichino i frutti della libera concorrenza»" ${ }^{89}$. Gli ostacoli all'unità sindacale hanno, secondo Rosselli, origini politiche e non economiche, e i motivi di divisione sono rinfocolati dalle imprese ${ }^{90}$. Ciò che viene definito come "universalizzazione dell'unionismo», piuttosto che causare alterazioni negative dei rapporti economici, condurrebbe a una regolarizzazione e a una azione ordinata del movimento sindacale, contrastando tendenze corporative e parassitarie ${ }^{91}$.

Ma una volta ribadita quella che Rosselli ritiene una corretta analisi delle funzioni del sindacato nella società capitalistica, subentrano una serie di considerazioni sull'ipotesi che il sindacato abbia la capacità di operare una vera trasformazione della struttura economica. E qui il tono diviene particolarmente pessimistico. La prima constatazione, per la verità già risultante da passate riflessioni, riguarda l'impossibilità del fronte sindacale di andare oltre l'unità rappresentativa e di realizzare un reale monopolio del lavoro ${ }^{92}$. Ciò non deriva da circostanze storiche sfavorevoli, né dall'incapacità della classe dirigente sindacale, bensì da un ineluttabile limite "costituzionale" del sindacato stesso. Quest'ultimo può infatti svolgere una funzione equilibratrice senza tuttavia essere in grado di mutare la struttura del mercato capitalistico. Se dall'azione rivendicativa sono scaturiti innegabili miglioramenti della classe lavoratrice sul fronte salariale e delle condizioni di lavoro, questa forza è anche il limite del sindacato. È il suo unico raggio d'azione, ed è anch'esso soggetto a notevoli condizionamenti. La pressione per ottenere l'aumento del reddito da lavoro ha infatti dei vincoli ben precisi, forzando i quali si rischiano per conseguenze «la fuga di capitali, l'emigrazione dell'industria, il sorgere di organizzazioni concorrenti, la contrazione della domanda, l'intervento della pubblica opinione» ${ }^{93}$. Quella stessa pressione può inoltre determinare fenomeni inflattivi che colpiscono indirettamente il potere d'acquisto dei salari ${ }^{94}$. Sul piano della politica dell'occupazione, poi, non sembra che il sindacato possa incidere significativamente, se è vero che Rosselli non ritiene efficace nemmeno lo strumento della diminuzione dell'orario lavorativo ${ }^{95}$. Ecco che il

${ }^{89}$ C. Rosselli, Monopolio e unità sindacale, «La Riforma sociale», settembre-ottobre 1924, ora in Id., Opere (vol. 1), cit., p. 259.

${ }^{90}$ Rosselli fa riferimento, in eterna polemica con Prato, alla creazione delle organizzazioni «gialle», al mantenimento dell'esercito dei disoccupati, agli accordi particolaristici e localistici. Cfr. C. Rosselli, Scienza economica e leghe operaie, cit., p. 226.

${ }^{91}$ Cfr. C. Rosselli, Miti liberistici o miti sindacali?, «La Riforma sociale», settembre-ottobre 1925, ora in Id., Opere (vol. 1), cit., pp. 306-307.

${ }^{92}$ Cfr. C. Rosselli, Monopolio e unità sindacale, cit., pp. 245-46.

${ }^{93}$ Ivi, p. 242.

${ }^{94}$ Cfr. C. Rosselli, L'azione sindacale ed i suoi limiti, «La Riforma sociale», novembre-dicembre 1925, ora in Id., Opere (vol. 1), p. 320.

${ }^{95}$ Cfr. C. Rosselli, Monopolio e unità sindacale, cit., pp. 239-240. 
sindacato risulta quale forza di interdizione, di correzione, ma inevitabilmente «variabile in funzione delle condizioni generali del mercato e della moderna impresa capitalistica $»^{96}$.

L'orientamento gildista contiene spunti critici di indiscutibile interesse per Rosselli. Il quale tuttavia non sembra mostrare un'eguale convinzione rispetto al modello teorico di cooperativismo integrale. Il progetto di Cole contempla una articolazione federativa, in cui le gilde (regolate da comitati direttivi eletti dai lavoratori) mantengono la propria autonomia e al contempo si coordinano tra loro (tramite un Parlamento delle gilde), risolvendo $i$ contrasti in ossequio alla funzionalità dell'intero sistema produttivo ${ }^{97}$. Rosselli non approfondisce lo studio di una ipotetica costituzione gildista. Pur convinto dalle intuizioni di Cole, rivela anzi scetticismo sulle reali possibilità pratiche di giungere a un socialismo democratico esclusivamente attraverso lo strumento sindacale. La conclusione cui giunge riguarda certo la riforma dell'ordine economico, ma non trascura il piano politico. Se il sindacato non può incidere più di tanto sulla creazione $\mathrm{e}$ la ripartizione di opportunità e redditi, è perché la natura stessa della sua funzione non lo consente. Il controllo del processo economico e della gestione dell'impresa è indispensabile per una concreta democratizzazione sociale oltre che politica, ma non può essere raggiunto esclusivamente per via sindacale ${ }^{98}$. Il sindacato risulta, adesso, necessario ma non sufficiente. La visione del socialismo continua a essere aperta, "sperimentalista", sottratta a concezioni organiche che preludano a esiti aconflittuali. Non è a caso che Rosselli rilancia il ruolo del partito e indica la strada di un ampliamento del consenso che coinvolga i ceti medi ${ }^{99}$. In retrospettiva,

${ }^{96}$ Ivi, p. 243.

${ }^{97}$ Cfr. G.D.H. Cole, Social Theory, in particolare il cap. 3, The Principle of Function, in cui si delinea una teoria dell'organizzazione sociale che, prendendo spunto dal contrattualismo rousseauviano, vede l'individuo unirsi in associazione con altri individui, su base volontaria, per il perseguimento di un obiettivo specifico, e, attraverso questa attività, svolgere un ruolo funzionale all'intero sistema, pp. 47-62. Si evidenzia qui quella che è stata definita «la visione bifocale» di Cole. La singola unità sociale è regolata da un rigido corporatismo rousseauviano. L'insieme della società è vista pluralisticamente e ottimisticamente, più in chiave liberale che socialista $(\mathrm{P}$. Beilharz, Labour's Utopia, cit., p. 78). In effetti, risulta evidente come in Cole venga sottovalutato il problema del coordinamento tra le diverse associazioni autonome (cfr. H.M. Magid, English Political Pluralism, AMS Press, New York 1966, p. 37).

${ }^{98}$ Cfr. C. Rosselli, L'azione sindacale ed i suoi limiti, cit., pp. 324-326 e 328-329.

${ }^{99}$ In un articolo da Londra dell'ottobre 1924 scrive: «questa ricchezza di medio ceto che si trova nelle fila del Labour Party [...] può spiegare molte differenze tra i nostri Parlamenti Socialisti continentali rigidamente classisti e il Labour Party, che preferisce di non insistere troppo in sede politica sul metodo della lotta di classe, mettendone però egregiamente in pratica i postulati quando si tratti di lottare in sede economica per la conquista di un livello più decente di vita per la classe operaia» (C. Rosselli, Laburisti e liberali faccia a faccia, «La Giustizia», 21 ottobre 1924, ora in appendice a S. Mastellone, Carlo Rosselli e «la rivoluzione liberale del socialismo», cit., p. 165). La critica del sistema di rappresentanza politica, in favore 
è questo un modo per ribadire i limiti culturali che hanno condizionato negativamente la politica socialista.

\section{3. «Il Quarto Stato»: l’unità nel segno di un socialismo democratico}

Dopo un periodo d'insegnamento universitario a Genova, Rosselli s'immerge sempre più nell'impegno politico, associando militanza ed elaborazione teorica. La rivista «Il Quarto Stato» nasce a Milano il 27 marzo $1926^{100}$. E il risultato della volontà comune di Nenni e Rosselli di costituire un laboratorio d'idee e perorare la causa della ricomposizione socialista dopo la sconfitta dell'opposizione aventiniana ${ }^{101}$. Anche in questa iniziativa, Rosselli tenta di sorreggere la proposta politica con la sollecitazione a un'analisi critica intransigente. C'è da fare i conti con la natura degli eventi storici dell'Italia prefascista, per rintracciare gli errori commessi, le ragioni delle divisioni, le possibili vie d'uscita ${ }^{102}$.

La visione rosselliana del periodo postunitario non si discosta molto dall'impostazione adottata da Salvemini e ripresa da Gobetti. L'Italia è vista come un "paese capitalisticamente arretrato, povero, disarticolato nelle sue parti, politicamente ineducato, affetto da provincialismo congenito $»^{103}$; e sono proprio questi gli elementi che hanno fatto da terreno ideale per l'edificazione del fascismo. Mussolini ha ratificato in regime la biografia della

di un tipo di rappresentanza "funzionale" è delineata da Cole soprattutto in Social Theory, cit., pp. 105-115.

${ }^{100}$ Rosselli dà notizia della prossima pubblicazione della rivista nella lettera alla madre del 25 gennaio 1926, in Epistolario Familiare, cit., p. 288.

${ }^{101}$ Nenni era direttore dell' «Avanti» ed aveva tentato di riavvicinare il PSI all'ala riformista espulsa nell'ottobre del 1922 . Un simile tentativo venne fatto nel dicembre del 1925 con una «lettera ai compagni», pubblicata sul giornale del partito il 12 dicembre 1925. Di fronte all'opposizione dei massimalisti, Nenni decise di lasciare la direzione. Nel febbraio-marzo 1926 fu lo stesso Rosselli ad invitare con una lettera Nenni a partecipare alla creazione di «Il Quarto Stato»; cfr. Appendice a N. Tranfaglia, Carlo Rosselli dall'interventismo a «Giustizia e Libertà», cit., pp. 369-71. Sull'esperienza de «Il Quarto Stato», si vedano l'introduzione di Domenico Zucàro al volume Il Quarto stato di Nenni e Rosselli, a cura dello stesso Zucàro, SugarCo, Milano 1977, in particolare le pp. 7-16; S. Merli, Il Quarto Stato di Rosselli e Nenni e la polemica sul rinnovamento socialista nel 1926, "Rivista storica del socialismo», Anno III, 1960, n. 11; P. Bagnoli, La battaglia socialista de "Il Quarto Stato", in Giustizia e Libertà nella lotta antifascista e nella storia d'Italia, cit., pp. 113-146 e A. Colombo, L'avventura di "Quarto Stato", in M. Degl'Innocenti (a cura di), Carlo Rosselli e il socialismo liberale, cit, pp. 57-64.

${ }^{102}$ Vedi lettera di Rosselli a Salvemini del 5 giugno 1926 in E. Signori (a cura di), Fra le righe. Carteggio fra Carlo Rosselli e Gaetano Salvemini, FrancoAngeli, Milano 2009, p. 110.

${ }^{103}$ C. Rosselli, Autocritica, «Il Quarto Stato», 3 aprile 1926, ora in Id., Opere (vol. 1), cit., p. 130. 
nazione. Il fascismo non è semplice reazione classista ${ }^{104}$, corrisponde piuttosto alla somma delle debolezze politiche, istituzionali e culturali dell'Italia liberale. La novità sta nella risposta al problema del "cos'è mancato?", sia dal punto di vista storico generale che da quello della concreta azione politica. Gobetti rintracciava nell'assenza di una vera rivoluzione liberale l'origine di tutti i mali; Rosselli matura e approfondisce sempre più l'idea che il vulnus sia consistito nel mancato compimento del sistema democratico. La fragilità delle istituzioni liberali è dunque associabile al difetto, o meglio all'assenza di un efficace e radicale processo di democratizzazione:

\section{[...] la libertà conquistata da esigue minoranze attraverso transazioni e rinunce restò estranea alla coscienza generale, dove si ebbero tutte le degenerazioni del sistema democratico parlamentare senza [...] una vera democrazia e un vero parlamento, dove al di là dello scenario di cartone della sovranità popolare il potere di fatto sempre risiedette nelle mani di una ristretta oligarchia facente capo al potere esecutivo, al partito di corte, alla burocrazia e a taluni gruppi plutocratici set- tentrionali, dove insomma difettarono le condizioni elementari per il sorgere e l'affermarsi di una salda coscienza politica ${ }^{105}$.}

La mancata realizzazione di riforme politiche di carattere generale, quali la «riforma tributaria, doganale, comunale, militare, elettorale» ${ }^{106}$ hanno determinato la debolezza endemica delle istituzioni e della società.

Da queste considerazioni Rosselli fa discendere la sua critica, o meglio la sua autocritica nei confronti sia del massimalismo sia del riformismo. Il giudizio sul massimalismo è sempre molto severo. L'accusa è d'ideologismo dogmatico, che si traduce in evanescenza dell'azione politica. L'intransigenza teorica ha immobilizzato il partito (lungamente a maggioranza

${ }^{104}$ Ivi, p. 129. In Contro il pessimismo, Rosselli ribadisce che «il fascismo è [...] l'Italia, il prodotto cioè di tutta la nostra storia, di tutta la nostra tradizione, la reale estrinsecazione dello stato economico e morale del paese, la logica conclusione di un lungo processo che andava lentamente maturando fin da prima della guerra» («Il Quarto Stato», 26 giugno 1926 ora in Id., Opere (vol. 1), cit., p. 154). E simile è anche il giudizio di Nenni, secondo il quale il fascismo non si può spiegare in termini di «semplice fenomeno di classe, ma come un fenomeno che trova la sua spiegazione nel complesso della vita sociale e morale del nostro paese» (P. Nenni, Chiarificazione, «Il Quarto Stato», 24 luglio 1926, ora in D. Zucàro (a cura di), Il Quarto stato di Nenni e Rosselli, cit., p. 113).

${ }^{105}$ C. Rosselli, Il congresso del PSLI, «Il Quarto Stato», 30 ottobre 1926, ora in Id., Opere (vol. 1), cit., p. 174.

${ }^{106}$ C. Rosselli, Autocritica, non demolizione, «Il Quarto Stato», 1 maggio 1926, ora in Id., Opere (vol. 1), cit., p. 140. Il giudizio di Rosselli è molto simile a quello espresso da Arturo Labriola sempre sulle pagine de «Il Quarto Stato». Labriola afferma che "chiunque è vissuto un po' dentro le cose politiche del nostro paese, sa quanto sia poco serio dire che le istituzioni politiche italiane, anche prima dell'avvento del fascismo, fossero le istituzioni di una democrazia», e aggiunge: «i mali d'Italia (provincialismo, spirito municipale, mancanza di senso civico, predominio 
massimalista), impedendogli di affrontare con efficacia i grandi dilemmi politici, primo tra tutti quello della "conquista del potere», in momenti in cui «un partito di opposizione della forza di quello socialista» doveva «essere pronto [...] a salire al potere ${ }^{107}$. Il nodo è nella concezione della democrazia, avversata dal purismo massimalista come "specchietto per le allodole" della borghesia. I massimalisti

[...] non sanno che farsene delle presenti istituzioni democratiche; e si danno alla pazza gioia quando esse sono demolite o minacciano di essere demolite; perché hanno la certezza che si avvicina così il giorno felice, in cui il "proletariato" prenderà il mestolo in mano e ricostruirà nuove istituzioni [...] intanto il "proletariato" perde tutte le possibilità di azione che le istituzioni democratiche tradizionali gli assicuravano ${ }^{108}$.

Rosselli non nasconde il proprio scetticismo sull'ipotesi di riunificazione socialista, sebbene riconosca l'effetto esiziale delle divisioni. Ė convinto che la scissione abbia «basi di una fragilità desolante» e non corrisponda alla volontà delle masse, che "ardentemente aspirano all'unità» ${ }^{109}$. Vede però nei massimalisti una suicida vocazione settaria, l'isolamento in una "torre d'avorio dogmatica" nel duplice ripudio della «degenerazione comunista» e del "collaborazionismo riformista» ${ }^{110}$.

Come si è visto, Rosselli è iscritto al Partito socialista unitario dal 1924. IL PSU, sciolto dal regime in seguito all'attentato Zaniboni, si era ricostituito come Partito socialista dei lavoratori italiani. Riformista secondo un'interpretazione volontaristica del socialismo ${ }^{111}$, egli è intransigente

delle oligarchie, delle camorre, incultura, criminalità, ecc.) sono nati dal persistere delle condizioni, che la democrazia vuol rimuovere [...] il rimedio non può trovarsi che in un sistema di assistenza della libera iniziativa del cittadino, nella organizzazione spontanea delle volontà e degli interessi dei cittadini stessi [...] ma che cosa è tutto questo se non la Democrazia, la vera libera e non sofisticata democrazia?» (A. Labriola, A rebours, "Il Quarto Stato», 17 aprile 1926, ora in D. Zucàro (a cura di), Il Quarto stato di Nenni e Rosselli, cit., rispettivamente p. 65 e pp.67-68).

${ }^{107}$ C. Rosselli, Autocritica, non demolizione, cit., pp. 143 e 138.

${ }^{108}$ C. Rosselli, Attenti alla nomenclatura!, «Il Quarto Stato», 31 luglio 1926, ora anche in S. Mastellone, Carlo Rosselli e la «rivoluzione liberale del socialsmo», cit., pp. 181-182.

${ }^{109}$ C. Rosselli, Il problema dell'unità socialista, Lettera aperta al direttore dell' "Avanti!", «Il Quarto Stato», 21 agosto 1926, ora in S. Mastellone, Carlo Rosselli $e$ «la rivoluzione liberale del socialismo», cit., p. 186.

${ }^{110}$ Cfr. Ivi, pp. 186-188. Per Rosselli l'unità socialista rappresentava uno dei tasselli per la composizione di un progetto politico antifascista, laddove per Nenni essa era la condizione imprescindibile per «l'unità della classe» e «l'unificazione della lotta di classe» (P. Nenni, Chiarificazione, «Il Quarto Stato», 24 luglio 1926, ora in D. Zucàro (a cura di), Il Quarto Stato di Nenni e Rosselli, cit., p. 117).

${ }^{111}$ Cfr. S. Neri Serneri, Democrazia e Stato. L'antifascismo liberaldemocratico e socialista dal 1923 al 1933, FrancoAngeli, Milano 1989, p. 183. 
dal punto di vista etico; pragmatico e aperto, viceversa, da quello ideologico. Da questa posizione ribadisce la sue critiche al riformismo turatiano, che pure ha avuto grande merito nella lotta per l'emancipazione della classe lavoratrice. La quale ha migliorato le proprie condizioni materiali e si è organizzata sindacalmente, ma non ha potuto evitare la sconfitta di fronte al fascismo. Per Rosselli ciò deriva da una sottovalutazione del problema nazionale come problema politico, culturale, morale. Nell'articolo Autocritica è sottolineato come, cadendo spesso in un grave determinismo economico, i riformisti non abbiano riconosciuto l'importanza della conquista di un regime di libertà civili e d'istituzioni democratiche in cui i socialisti potessero identificarsi, così che «la libertà italiana» fu «figlia di transazioni, di adattamenti e di taciti accomodamenti», e «il suffragio universale apparve una gratuita concessione e non una conquista cosciente». Il partito socialista, in quanto non valutò nel giusto valore «il problema politico, fu travolto dalla strepitosa vittoria del 1900 [...] che di fatto interessò solo le aristocrazie operaie del nord [...] e non seppe condurre dopo il '900 la grande battaglia per le libertà e le fondamentali conquiste politiche in nome ed in pro dell'intero proletariato", degenerando troppo spesso nel corporativismo e «barattando i valori supremi per il classico piatto di lenticchie abilmente presentato da Giolitti» ${ }^{112}$.

Il giudizio è molto duro, forse ingeneroso, e pronta è la replica di Claudio Treves su «Critica sociale», dal titolo Autocritica o demolizione?. Treves ha gioco facile nel rivendicare la necessità storica di subordinare l'emancipazione politica a quella economica, poiché le basi morali,

[...] se non sono state messe ancora oggi, dopo quarant'anni di elevazione economica delle plebi - ancora meno si sarebbero mai potute porre con un proletariato agricolo che guadagnava duecentocinquanta lire all'anno, lavorava dall'alba alla notte, ignorava l'alfabeto e crepava di pellagra ${ }^{113}$.

Nega la mancanza di determinazione, tra i riformisti, nell'indicare l'obiettivo della presa del potere «non per l'attuazione massimalista e classista di un socialismo totalitario soltanto nelle belliche macerie, ma per la tutela delle sue libertà» ${ }^{114}$. Le responsabilità della «distruzione della forza politica del socialismo», più che alla «inclinazione ai comodi compromessi utilitari», è attribuita quindi «alle irruenze del più spinto idealismo rivoluzionario $»^{115}$. Tale tipo di difesa d'ufficio sembra però animata da profonda incomprensione rispetto al punto centrale del discorso rosselliano. Là do-

${ }^{112}$ C. Rosselli, Autocritica, cit., p. 131.

${ }^{113}$ C. Treves, Autocritica o demolizione?, «Critica Sociale», anno XXXVI, N. 7, 1-15 aprile 1926, p. 93 (firmato Rabano Mauro).

${ }^{114}$ Ivi, p. 95.

115 Ivi, p. 94. 
ve Rosselli denunciava il mancato compimento di un sistema istituzionale e politico di democrazia, in cui anche il proletariato potesse riconoscersi, Treves risponde: «in che siamo battuti? Nella nostra ideologia socialista no», chiarendo subito la sua interpretazione ambigua delle istituzioni col dire: «dunque noi siamo stati battuti in qualche cosa che non era "noi", sebbene fosse anche di noi. Noi siamo stati battuti nel liberalismo, nella democrazia, che essendo "noi", era però più propriamente la borghesia»"116.

Su questo tema la distanza, ideologica prima che politica, è enorme. Nella sua controreplica, dal titolo Autocritica, non demolizione, Rosselli insiste sulla democrazia come valore e non come strumento:

[...] ma non vi sembra, Rabano Mauro, che il fronte della civiltà liberale democratica sia proprio caduto a tutto danno morale e materiale (non siamo mistici...) del proletariato e con nessun danno (anzi...) della borghesia capitalistica? [...] Siete forse voi ancora un credente in quella facile e superata formula (superata dai fatti) che fa della democrazia e del capitalismo due sinonimi, due aspetti necessariamente legati nel mondo a civiltà occidentale? ${ }^{117}$.

Le critiche di Rosselli possono apparire forzate. Difficilmente, visto il contesto nazionale e i rapporti di forza esistenti (soprattutto all'interno del partito socialista tra riformisti e massimalisti), il "percorso della lotta" avrebbe potuto svilupparsi in modo diverso ${ }^{118}$. Il giudizio rosselliano andrebbe però letto secondo una prospettiva rivolta al futuro; come il tentativo di aprire uno squarcio tra l'inconcludenza del rivoluzionarismo e le secche del riformismo-giolittismo, per la modernizzazione politica, istituzionale e culturale del paese ${ }^{119}$ : un tentativo coraggioso (o se si vuole

${ }^{116}$ Ivi, p. 95.

${ }^{117}$ C. Rosselli, Autocritica, non demolizione, cit., pp. 135-36. Nello stesso articolo Rosselli esemplifica la sua critica con riferimento alla lotta per il suffragio universale, che, da "premessa imprescindibile per l'avviamento ad una reale democrazia», fu considerato non un fine, "ma un mezzo, un semplice mezzo neppure indispensabile per "agevolare" la conquista [...] di quella legislazione sociale che parve [...] potesse condurre alla progressiva erosione delle basi del regime», e tutto questo in un paese in cui «le masse furono lontane sempre dalle grandi lotte politiche, [...] non si ebbero mai i grandi conflitti costituzionali, [...] dove v'era pure bisogno di fare acquistare alle masse, attraverso una grande e pacifica rivoluzione, una coscienza politica» (ivi, pp. 141-142).

${ }^{118}$ Per un quadro efficace della storia socialista dei primi anni '20, in particolare riguardo ai rapporti tra massimalisti, riformisti e comunisti, cfr. i capp. IV (1920-1926. La disfatta del vecchio e del nuovo massimalismo) e V (La battaglia non combattuta per la difesa della democrazia: la ragione senza storia e la storia senza ragione) del volume di M.L. Salvadori La sinistra nella storia d'Italia, cit.

${ }^{119}$ In questo senso cfr. P. Bagnoli, La battaglia socialista de «Il Quarto Stato», in Giustizia e libertà nella lotta antifascista e nella storia d'Italia, cit., pp. 123-125, che, riguardo alla polemica Rosselli-Treves parla di «visione dinamica della democrazia» del primo, rispetto a una «visione difensiva» del secondo. 
coraggiosamente velleitario) di simulare la transitorietà del fascismo proprio nel momento della sua compiuta trasformazione in dittatura, e immaginare un ricominciamento che escluda le tare del passato ${ }^{120}$.

Appare chiaro, a questo punto, che il fulcro dottrinario del pensiero di Rosselli vada rintracciato in quell'asse che lega liberalismo, democrazia e socialismo, con la democrazia non a caso volta a ricoprire la posizione mediana, perché dimensione irrinunciabile per dare alla libertà una realizzazione sociale. Il dibattito sul rapporto socialismo-democrazia coinvolge del resto in quegli anni molti esponenti della sinistra italiana, e «Il Quarto Stato» è luogo di interventi interessanti ${ }^{121}$. A un'attenta lettura, la posizione di Rosselli si distingue dalle altre per prospettiva interpretativa e conclusioni. Nell'articolo Attenti alla nomenclatura!, liberalismo e democrazia vengono considerate nelle diverse accezioni, ideologica e istituzionale. Se l'ideale liberale si è tradotto in «libertà di pensiero, libertà di stampa, libertà di associazione, libertà di riunione, regime rappresentativo», vale a dire nelle tipiche istituzioni liberali, la democrazia esige «che tutti i cittadini abbiano gli stessi diritti di libertà e che non vi sia più nessun privilegio: il suffragio universale è la istituzione tipica della democrazia» ${ }^{122}$. E nel quadro delle istituzione politiche liberali e democratiche si inserisce l'istanza socialista, così esemplificata: «voi potete limitarvi a domandare l'abolizione dei privilegi politici, e potete domandare l'abolizione dei privilegi economici [...] questa forma di "democrazia economica" è il socialismo»" ${ }^{123}$.

Socialismo "dentro" la democrazia e non democrazia mero strumento del socialismo, dunque. Nenni, che pure condivide con Rosselli una visione non settaria del socialismo e la necessità di un fronte comune antifascista, offre una lettura diversa della democrazia. Contro le posizioni massimaliste e soprattutto comuniste, Nenni afferma che le «istituzioni democratiche costituiscono [...] una esigenza fondamentale per il movimento socialista» e che "nessun grande movimento di masse è possibile senza democrazia politica ${ }^{124}$. Ma questa difesa non ha altro obiettivo

${ }^{120}$ Rosselli era indubbiamente animato da un irriducibile ottimismo. A proposito dell'esperienza di «Il Quarto Stato», scriveva a Salvemini il 5 giugno del $1926 \mathrm{da}$ Milano: «Se ci lasciano un po' di libertà per un anno ancora, ci metteremo in grado di dominare o per lo meno di influenzare potentemente l'opposizione o quel simulacro di opposizione ancora in piedi» (in Fra le righe. Carteggio fra Carlo Rosselli e Gaetano Salvemini, cit., p. 110).

${ }^{121}$ Per un'analisi dei diversi contributi che animarono il dibattito su «Il Quarto Stato» cfr. N. Tranfaglia, Carlo Rosselli dall'interventismo a "Giustizia e libertà», cit., pp. 285-87 e 300-01; S. Mastellone, Carlo Rosselli e «la rivoluzione liberale del socialismo», cit., pp. 59-63; P. Bagnoli, La battaglia socialista de «Il Quarto Stato», cit., pp. 128-146.

${ }^{122}$ C. Rosselli, Attenti alla nomenclatura!, cit., p. 182.

123 Ibid.

${ }^{124}$ P. Nenni, La crisi della democrazia, «Il Quarto Stato», 3 luglio 1926, ora in D. Zucàro (a cura di), Il Quarto Stato di Nenni e Rosselli, cit., pp. 105-106. 
che alimentare la lotta di classe: «la democrazia politica non è [...] che una tappa. Nel momento stesso in cui il proletariato socialista la conquista, esso dialetticamente la nega». La dialettica classista del marxismo rende le istituzioni democratiche strumentali, nel senso che "per alcuni partiti la democrazia politica è il fine, per noi essa non è che il mezzo e neppure il solo mezzo, per realizzare la democrazia economica» ${ }^{125}$. Si potrebbe dire che sono i soliti "conti con Marx" a non tornare. Da un lato, sta una coscienza sinceramente democratica, come sempre sarà in Nenni, dall'altro, l'inevitabile ambiguità teorica conseguente al fatto che Nenni identifica comunque nel marxismo l'unica e sola dottrina del proletariato, fino alla conclusione che «l'ideologia democratica è la negazione della lotta di classe, anche quando afferma il fatto della lotta di classe $»^{126}$.

$\mathrm{Su}$ «Il Quarto Stato», il dibattito sul valore della democrazia è ampio il regime fascista si sta edificando sulle macerie del parlamento -, e il filo conduttore sembra essere la ricerca di nuove formule che possano rilanciare le sorti del socialismo. Degna di interesse, in particolare, è la posizione di Giuseppe Saragat, che propone una lettura integralmente democratica di Marx. L'errore dei massimalisti sarebbe stato quello di ripudiare «la democrazia come un'espressione dell'ideologia borghese ${ }^{127}$, mentre il riformismo identificava «troppo facilmente la pseudo-democrazia di Giolitti con la compiuta democrazia integrale ${ }^{128}$. Quest'ultima si costruisce invece, secondo Saragat, attraverso uno spirito di reale autonomia politica, che «rende cosciente l'operaio dei suoi diritti e quindi lo arma del senso di classe perché gli conferisce quel tanto di umanità necessaria per renderlo sensibile all'ingiustizia da cui è umiliato» ${ }^{129}$.

Altri interventi si susseguono, sempre su «Il Quarto Stato». Secondo Adelchi Baratono, la democrazia deve farsi socialista «cercando il proprio fondamento non nel valore astratto dell'individuo ma nella funzione sociale del lavoro» ${ }^{130}$, mentre, in virtù di un concezione idealistica del marxismo, Rodolfo Morandi afferma che «la democrazia del socialismo non può avere nulla in sé che la confonda con la democrazia degli antichi

${ }^{125}$ P. Nenni, La politica socialista, «Il Quarto Stato», 10 aprile 1926, ora ivi, pp. 61-62. La differenza tra Nenni e Rosselli dal punto di vista teorico è notevole e non sembra sia stata messa sufficientemente in luce dalla letteratura critica. Tranfaglia d'altronde ha indicato come fossero gli interessi di strategia politica a unire i due, interessi che in quel particolare momento storico risultavano ad entrambi prioritari rispetto all'analisi ideologica (N. Tranfaglia, Carlo Rosselli dall'interventismo a «Giustizia e libertà», cit., p. 283).

${ }^{126}$ P. Nenni, La crisi della democrazia, cit., p. 105.

${ }^{127}$ G. Saragat, Il nostro errore, "Il Quarto Stato», 22 maggio 1926, ora in D. Zucàro (a cura di), Il Quarto Stato di Nenni e Rosselli, cit., p. 81.

${ }^{128}$ Ivi, p. 83.

${ }^{129}$ Ivi, pp. 82-83.

${ }^{130}$ A. Baratono, L'unità, punto d'arrivo, «Il Quarto Stato», 7 agosto 1926, ora ivi, p. 189. 
o con quella borghese» ${ }^{131}$. C'è la ricerca di vie nuove, ma allo stesso tempo un ondeggiare tra visioni che, da ottiche diverse, cercano uno sbocco in democrazie-altre, legittimate entro una cornice di marxismo più $o$ meno ibridato ${ }^{132}$. Il problema rimane quello di capire quale sia il valore, e non solo la funzione, da attribuire alla democrazia. In altri termini, se la democrazia è un mezzo per raggiungere il fine del socialismo - in antitesi all'ipotesi rivoluzionaria dei massimalisti e sovietica dei comunisti -, cosa sarà di tale mezzo quando il fine sarà conquistato? E soprattutto, come si regolerà allora la maggioranza proletaria nei confronti di una minoranza che non volesse accettare il sistema socialista ${ }^{133}$ La risposta di Rosselli prescinde consapevolmente da vincoli di prospettiva classista e palingenetica. Fedele a una visione liberale e conflittuale della storia, egli crede nella democrazia, non solo come insieme d'istituzioni che permettano di raggiungere il potere tramite la legge della maggioranza, bensì come perimetro di garanzie per tutti, in cui il socialismo può farsi asintoticamente, venendo continuamente contrastato, senza mai cristallizzarsi in sistema definitivo. Non altra è perciò la conclusione:

[...] dove ci sono istituzioni liberali e democratiche le difendo con tutte le mie forze, anche se imperfette, perché esse mi servono di strumento per conquistare ulteriori condizioni politiche in cui si realizzi sempre meglio il mio ideale di libertà e di democrazia ${ }^{134}$.

${ }^{131}$ In quanto, prosegue Morandi, «in quelle prime l'antitesi di governante e governato, che si basa veramente su di una distinzione logica, permane immanente all'individuo, che è governato non dal suo voto - in quanto diventa col voto governante - ma da una risultanza numerica astratta di voti, epperò non dalla sua volontà, ma da una concorrenza di volontà in una volontà fittizia e irreale» (R. Morandi, La democrazia del socialismo, «Il Quarto Stato», 5 giugno 1926, ora ivi, pp. 177-78).

${ }^{132}$ A questo proposito nella rivista di Nenni e Rosselli si sviluppa un dibattito sulle diverse interpretazioni del marxismo. Vedi in particolare, oltre il succitato articolo di R. Morandi, Socialismo e idealismo di Lelio Basso, 3 aprile 1926 e 10 aprile 1926, firmato Prometeo Filodemo, ora ivi, pp. 267-279; Problemi filosofici del socialismo di Santino Caramella, 17 aprile; Socialismo e kantismo di Alfredo Paggi, 8 maggio; Socialismo e idealismo di Antonio Grespi, 5 giugno; Il valore del socialismo di Guido da Ferrara, 7 agosto, tutti pubblicati sulle pagine de «Il Quarto Stato». A cercare un punto d'equilibrio, per un'autentica interpretazione di Marx, è ancora una volta Rodolfo Mondolfo con tre articoli significativi: Né materialismo né idealismo ma realismo critico pratico, del 17 aprile, ora in $\mathrm{D}$. Zucàro (a cura di), Il Quarto Stato di Nenni e Rosselli, cit., pp. 280-284 e in R. Mondolfo, Umanismo di Marx, cit., pp. 260-265; Per la revisione del bilancio idealistico, 21 agosto, ora ivi, pp. 266-73; Primum intelligere, 23 ottobre, ora ivi, pp. 274-276.

${ }^{133} \mathrm{Su}$ questo argomento sono di particolare interesse i rilievi di D. Settembrini, Socialismo marxista e socialismo liberale, "Nuova serie di quaderni di Mondoperaio», 4, 1976, pp. 63-72.

${ }^{134}$ C. Rosselli, Attenti alla nomenclatura!, cit., p. 183. Le libertà democratiche sono quindi un patrimonio irrinunciabile, che va difeso, anche con il ricorso alla violenza, quando esse vengano negate. Rosselli afferma infatti che «dove le istituzioni politiche sono liberali e democratiche, io sto per il metodo della propaganda 
Dunque, Rosselli tenta di rendersi interprete di una via originale al riformismo, che, se ideologicamente flessibile, è non di meno radicale sotto il profilo del progetto politico. Sulla base di un volontarismo non filosofico - «qui non si tratta, per fortuna, né di positivismo né d’idealismo. Mi riferisco all'azione e dico che nell'azione non si può essere che dei volontaristi ${ }^{135}$-, propone un'alleanza che non abbia come collante la sola componente negativa dell'antifascismo, ma che guardi anche alla costruzione di una democrazia progressiva ${ }^{136}$. Vengono messe a tacere le pregiudiziali ideologiche e proclamata «la necessità del più ampio mobilismo tattico» ${ }^{137}$. La visione del partito è certo antidogmatica, e ciò in contrasto con la tradizione continentale socialista ${ }^{138}$; il partito non è «un feticcio; siamo abbastanza sensibili per capire che non saranno le etichette che in definitiva trionferanno, ma le opere» ${ }^{139}$. E proprio sul piano delle «opere», degli obiettivi, si esprime il massimo della risolutezza. Per la conquista di un sistema di «integrale e agguerrita democrazia» ${ }^{140}$, è indispensabile non guardare indietro. Bisogna andare oltre il fascismo, ma anche oltre «l'italietta di Giolitti e di Facta» ${ }^{141}$, ponendo la questione istituzionale ${ }^{142}$. Il compito di

liberale e delle trasformazioni pacifiche. Ove mancano le istituzioni liberali e democratiche, io sono costretto ad adottare il metodo rivoluzionario [...] per passare ad un regime di liberalismo e di democrazia politica» (Ibid.). La posizione di Rosselli ha una coerenza a cui non fa giustizia l'affermazione di Tranfaglia secondo cui permarrebbe un'oscillazione «tra il recupero dell'interpretazione mondolfiana di Marx e [...] il rifiuto integrale del marxismo» (N. Tranfaglia, Carlo Rosselli dall'interventismo a "Giustizia e Libertà», cit., p. 293). Sarebbe forse più opportuno parlare di un Rosselli che non si pone né con Marx né contro Marx, ma oltre il marxismo, quantomeno considerato come sola e legittima ideologia del socialismo.

${ }^{135}$ C. Rosselli, Volontarismo, «Il Quarto Stato», 12 giugno 1926, ora in Id., Opere, cit., p. 146. In una lettera da Milano del 15 aprile 1926, Rosselli si lamenta con la madre del fatto che «in Italia tutti son buoni a parlare di questioni filosofiche e politiche generali; nessuno di questioni concrete», (Epistolario Familiare, cit., p. 292).

${ }^{136}$ Nadia Urbinati ha affermato che: «Rosselli interpretò l'antifascismo come democrazia e quindi secondo una prospettiva positiva, cioè dal punto di vista del dopo-fascismo; per costruire, non semplicemente per negare. Egli non era democratico perché antifascista; era antifascista perché democratico» (N. Urbinati, Il "Socialismo Liberale" e la democrazia oggi, "Quaderni del Circolo Rosselli», nuova serie, 1/2000, p. 47).

${ }_{137}$ C. Rosselli, Un congresso ed $i$ suoi problemi, «Il Quarto Stato», 9 ottobre 1926, ora in Id., Opere (vol. 1), cit., p. 169.

${ }^{138}$ All'articolo Un congresso e i suoi problemi fa riferimento Mastellone, individuando un implicito riferimento alla forma organizzativa del laburismo inglese, cfr. S. Mastellone, Il partito politico nel Socialismo liberale di Carlo Rosselli, cit., in particolare le pp. 116-17.

${ }^{139}$ C. Rosselli, Un congresso ed i suoi problemi, cit., p. 168.

${ }^{140}$ Ivi, p. 171.

${ }^{141}$ C. Rosselli, Contro il pessimismo, cit., p. 159.

${ }^{142}$ Rosselli cita la mozione del congresso del partito socialista dei lavoratori italiani, che in prima persona aveva contribuito a redigere, in cui è scritto che «la 
aggregare una coalizione, unita dalla concretezza di un progetto di azione politica senza compromessi, e non da vincoli ideologici, spetta ai socialisti. Al fianco di questi, Rosselli vede i repubblicani e le «scarse pattuglie democratiche», per la creazione di un'alleanza da contrapporre alla «doppia concezione dittatoriale, per quanto profondamente diversa nei fini», del fascismo e del comunismo ${ }^{143}$. Per sconfiggere il fascismo è necessario partire da un comune denominatore antitotalitario, nella speranza che questa premessa inneschi più larghe convergenze, «quando all'interno di ciascun gruppo, di ciascun partito sia avvenuta quella definitiva chiarificazione che è un'indispensabile premessa all'azione e quando si constati una fondamentale unicità di vedute» ${ }^{144}$.

L’esperienza de «Il Quarto Stato» racchiude l'ultimo sussulto di fiducia nella possibilità di far cadere il fascismo attraverso un ricompattamento dell'opposizione. Con gli occhi del poi, si tratta di un'illusione irrealistica. Nel 1926, il fronte antifascista - peraltro nella versione "ridotta" auspicata da Rosselli ${ }^{145}$ - è lontano da concrete vocazioni unitarie e il regime è in via di rapido consolidamento. Tuttavia, le riflessioni di Rosselli assumono consistenza se assunte in prospettiva. La loro validità è sicuramente più incisiva nel tempo lungo delle idee che in quello contingente dei fatti.

crisi scatenata dal fascismo, essendo nel più alto senso istituzionale e riproponendo quindi tutti i problemi della vita dello Stato, non si supererà con un semplice ritorno allo statu quo ante» (C. Rosselli, Il congresso del partito socialista dei lavoratori italiani, cit., p. 173). Sulla questione istituzionale Nenni aveva affermato: «al punto in cui siamo, non è possibile concepire una virile opposizione al fascismo fuori del terreno repubblicano» (P. Nenni, Chiarificazione, cit., p. 117). Per Nino Levi: «pregiudiziale repubblicana significa negazione del valore democratico dell'Italia di ieri» (Unità socialista e pregiudiziale repubblicana, "Il Quarto Stato», 4 settembre 1926, ora in D. Zucàro (a cura di), Il Quarto Stato di Nenni e Rosselli, cit., p. 202).

${ }^{143}$ Nei confronti del comunismo italiano Rosselli assume un atteggiamento liquidatorio. I riferimenti sono rarissimi e i giudizi molto schematici. L'unica spiegazione, per quanto parziale, è rintracciabile nelle parole di Garosci, quando afferma che l'«avversione al comunismo proveniva più che da uno studio e da una critica specifica, parte da istinto di avversione alla violenza massimalista, parte dall'amore istintivo [...] per la libertà, per le istituzioni democratiche» (A. Garosci, Vita di Carlo Rosselli, cit., p. 61). Ciuffoletti ha sostenuto che «secondo Rosselli, fascisti e comunisti avevano in comune sia l'avversione al liberalismo che alla democrazia» (Z. Ciuffoletti, Contro lo statalismo, cit., p. 97).

${ }^{144}$ C. Rosselli, Contro il pessimismo, cit., p. 160.

${ }^{145}$ Versione che avrebbe dovuto sollecitare la «convergenza di una liberaldemocrazia fattasi risolutamente repubblicana e un socialismo purgato dal determinismo e dal verbalismo inconcludente» (L. Rapone, L'Italia antifascista, in G. Sabbatucci e V. Vidotto (a cura di), Storia d'Italia, IV, Guerra e fascismo, Laterza, Roma-Bari 1998, p. 516. 


\title{
CAPITOLO QUARTO
}

\author{
SOCIALISMO LIBERALE
}

\section{Marxismo e revisionismo}

Con l'approvazione delle leggi fascistissime il governo di Mussolini procede nella trasformazione dello Stato liberale in dittatura. Le opposizioni politiche e sindacali ne escono annichilite e le libertà di associazione e di stampa sono gravemente compromesse. L'antifascismo è sotto scacco. Prevalgono il disorientamento e l'impotenza. Rosselli si espone sempre di più nell'impegno clandestino contro il regime, pagandone personalmente le conseguenze.

Socialismo liberale è scritto nel biennio 1928-29 a Lipari. Sono gli anni del confino, conseguente alla condanna subita per aver organizzato, con Ferruccio Parri e Sandro Pertini, l'espatrio clandestino di Turati dall'Italia ${ }^{1}$. Il volume che verrà pubblicato a Parigi nel 1930 - dopo la fuga avventurosa dall'isola eoliana con Emilio Lussu e Francesco Fausto Nitti ${ }^{2}$ - è il primo tentativo, e rimarrà l'ultimo, di definizione sistematica di un pensiero che sistematico non sarà mai. Esso rappresenta il compimento di un percorso di elaborazione dottrinale e di impegno politico insieme, che non può quindi risolversi esclusivamente sul piano teorico, ma che esprime un progetto e prefigura una filosofia della prassi. Socialismo liberale è al tempo stesso il bilancio intellettuale di un uomo che vive una parentesi di inazione forzata e la sfida concreta di un antifascista che cerca di guardare a un orizzonte di lungo periodo.

L'opera può schematicamente dividersi in due parti. Una parte critica che rimanda al dibattito sul revisionismo, e una parte propositiva, che indica esplicitamente come meta il superamento del marxismo e l'approdo a un socialismo liberale ${ }^{3}$. Sono i temi affrontati frammen-

${ }^{1}$ Sulle vicende riguardanti la fuga di Turati e il conseguente processo svoltosi a Savona, cfr. A. Garosci, Vita di Carlo Rosselli, cit., pp. 77-108 e N. Tranfaglia, Carlo Rosselli dall'interventismo a "Giustizia e libertà», cit., pp. 343-59.

${ }^{2}$ Cfr. L. Di Vito, M. Gialdroni, Lipari 1929. Fuga dal confino, Laterza, RomaBari 2009.

${ }^{3}$ Cfr. N. Bobbio, Introduzione a C. Rosselli, Socialismo liberale, Einaudi, Torino 1997, p. XXI.

Carmelo Calabrò, Liberalismo, democrazia, socialismo. L'itinerario di Carlo Rosselli, ISBN 978-88-6453-084-0 (print), ISBN 978-88-6453-086-4 (online)

(c) 2009 Firenze University Press 
tariamente nella produzione che abbiamo esaminato. Ora essi trovano esposizione più articolata e compiuta, con riferimenti nuovi e maggiore coerenza discorsiva. Soprattutto, Socialismo liberale ha valore di sintesi e al contempo di spartiacque. È un consuntivo che chiude una fase speculativa e politica.

Il libro prende avvio dalla formulazione di un giudizio drastico sulla dottrina di Marx quale concezione del mondo, filosofia della storia, insieme organico inscindibile. Rosselli accentua la riconduzione del marxismo alla versione che reputa dominante e che coincide con un rigido determinismo economico. Ne deriva uno schema polemico che indulge al sillogismo. Alla stregua delle coordinate marxiste, l'intero sviluppo storico sarebbe figlio di una necessità incoercibile, esito delle contraddizioni «tra le forze espansive della produzione e le forze conservatrici simbolizzate dai preesistenti rapporti sociali» ${ }^{4}$. La conoscenza delle leggi che presiedono a tali contraddizioni permetterebbe di descrivere il moto della storia e di prevederne "scientificamente" lo svolgimento. Per Rosselli, è da questo nucleo che discendono in Marx, a guisa di postulati, i concetti teorici utili a spiegare e prevedere l'avvento del socialismo. Il socialismo si compirebbe difatti in virtù «di un processo obbiettivo e fatale di trasformazione di $\operatorname{cose} »^{5}$, risultato appunto della contraddizione capitalistica tra forze e forme di produzione. La lotta di classe, dinamica che conduce concretamente al socialismo, altro non sarebbe che un riflesso «del contrasto esistente nelle cose stesse; la faccia umana della dialettica immanente nelle cose ${ }^{6}$.

Assunto per vero tale presupposto, nel marxismo come sistema non può esserci posto per il momento della volontà; nella storia che si compie per necessità, anche il socialismo non può sfuggire a questa regola. E non altra è la ragione dell'influenza storica del marxismo sul movimento socialista. Rosselli spiega la straordinaria potenza persuasiva dell'opera di Marx proprio in rapporto alla funzione scientifica che il riferimento alla necessità pretendeva di svolgere. L'ineluttabilità dell'avvento del socialismo si trasformava, con la propaganda, in una fede, ancora più salda perché poggiante su una previsione razionale. Con riguardo specifico al Manifesto, Rosselli afferma appunto che «nulla è più drammatico di quella sua volutamente fredda analisi del sistema capitalistico di sfruttamento che termina con la visione della catastrofe inevitabile dalla quale solo potrà sortire la società nuova [...] un sogno romantico in nome della ragione! La giustizia alleata con la scienza, anzi la scienza che è di per se giustizia! ${ }^{7}$. Più che sarcasmo, c'è in questa frase l'intelligenza del potere seduttivo di un'ideologia non così facilmente sostituibile.

\footnotetext{
${ }^{4}$ C. Rosselli, Socialismo liberale, cit., p. 6.

${ }^{5}$ Ivi, p. 10.

${ }^{6}$ Ivi, p. 12.

${ }^{7}$ Ivi, p. 17.
} 
Sennonché ogni scienza si basa su previsioni, e, quando esse si rivelano erronee, è la scienza stessa a uscirne pregiudicata. Il marxismo aveva profetizzato il passaggio dalla società borghese a quella socialista in nome del catastrofismo legato alle tendenze dell'immiserimento progressivo, della proletarizzazione, della concentrazione di ricchezza; tendenze (o, meglio, leggi) che avrebbero dovuto generare l'implosione dell'economia capitalistica. Rosselli ha buon gioco a ripetere, sulle orme dei revisionisti, che tutto ciò non si è avverato, rilevando un primo momento di crisi del marxismo proprio nell'avanzata del movimento operaio alla fine dell' 800 . La prassi politica ha smentito la teoria, la classe lavoratrice si è sindacalizzata avviando un moto d'emancipazione sulla base della possibilità «e desiderabilità di una trasformazione graduale della società borghese con le armi del voto, della contrattazione, dell'agitazione, cioè col ricorso al metodo democratico» ${ }^{8}$.

Il revisionismo, dunque, che secondo Rosselli sorge come inevitabile tentativo di riconciliare teoria e prassi. E che nasce per dare spazio all'elemento volontaristico. L'intenzione è di interpretare Marx, non di tradirne lo spirito. Rosselli si riferisce innanzitutto all'opera di Eduard Bernstein, che avrebbe voluto "mantenersi interna al sistema e procedere cautamente [...] per sostituire al Marx tutto angoli e spigoli della tradizione ortodossa, un Marx più complesso ed umano» ${ }^{9}$. In effetti Bernstein, partendo dall'assunto dell'assoluta scientificità del materialismo storico e credendo di poter reinterpretare alcuni concetti a suo parere secondari, aveva valorizzato, e comunque emancipato dalla condizione di semplici "riflessi" della struttura, le variabili cosiddette sovrastrutturali. Rosselli cita il passo bernsteiniano in cui è espressa la necessità «di rendere piena ragione, accanto alle forze e ai rapporti produttivi, alle idee di diritto e di morale, alle tradizioni storiche e religiose, agli influssi geografici, a quelli della natura in cui rientrano [...] le tendenze spirituali dell'uomo» $»^{10}$. Proprio il mancato avverarsi delle previsioni economiche di Marx spingeva a legittimare la prassi del movimento operaio, che, specie nei paesi più

${ }^{8}$ Ivi, p. 20. Il fatto che il movimento operaio stesse smentendo, con la sua prassi storica, le premesse teoriche del marxismo, condusse i partiti a "conciliare l'inconciliabile: cioè il momento pratico col teoretico, il semplicismo messianico del loro programma finalistico con le concrete rivendicazioni sindacali, la tattica rivoluzionaria e la pratica intransigente della lotta di classe, con i quotidiani fenomeni di transazione e di collaborazione dei sindacati» (ivi, p. 19). Si può notare come Rosselli leghi insieme analisi dottrinale e giudizio politico. Bobbio ha sottolineato che, in tutta l'opera, «la critica della teoria nasce dalla critica della prassi, e a sua volta sfocia in una nuova teoria, più correttamente in un abbozzo di nuova teoria, che si presenta come ispiratrice di una nuova prassi» (N. Bobbio, Introduzione a C. Rosselli, Socialismo liberale, cit., p. XXX).

${ }^{9}$ C. Rosselli, Socialismo liberale, cit., p. 23.

${ }^{10}$ Ivi, p. 25. Cfr. E. Bernstein, I presupposti del socialismo e i compiti della socialdemocrazia, Laterza, Bari 1968, pp. 34-35 (ed. orig. 1899). 
avanzati, era frutto di una spinta di volontà più che di una attesa messianica $^{11}$. Ma, con la presa d'atto di tale mutamento, veniva secondo Rosselli demolito l'intero apparato determinista. Il catastrofismo non solo non si realizzava nei fatti, ma non era neanche auspicabile. Bernstein affermava infatti l'utilità della via gradualista, democratica ${ }^{12}$. Dalla legittimazione della prassi alla negazione del principio filosofico il passo era breve: il successo del socialismo non dipendeva più «dalla sua "necessità economica immanente"», e non era "né possibile né necessario dargli un fondamento puramente materialistico» $»^{13}$. Rosselli considera la rottura di Bernstein un punto di non ritorno. Una rottura che scatena la decisa reazione dell'ortodossia, eppure, per usare un parallelo con la storia della spiritualità cristiana $^{14}$, il germe dell'eresia diviene inarrestabile: revisionismo di destra e di sinistra imperversano, e le interpretazioni del marxismo si moltiplicano divaricandosi ${ }^{15}$.

È questa la via seguita nell'analisi più specifica del rapporto tra marxismo e revisionismo in Italia ${ }^{16}$. Nel nostro paese, la dottrina di Marx aveva avuto in Antonio Labriola il primo, autorevole interprete. Definito da Rosselli «l'apologista del materialismo storico» ${ }^{17}$, Labriola aveva rivendicato una lettura ortodossa del marxismo, da contrapporre a ogni sorta di visione unilaterale. Contro la riduzione del metodo marxista d'interpretazione storica a gretto materialismo, nella riflessione di Labriola il rapporto struttura-sovrastruttura si spiegava non in termini di derivazione meccanica, ma come processo «complicato, spesso sottile e tortuoso, non sempre decifrabile» ${ }^{18}$. In Labriola, la concezione articolata e complessa

${ }^{11}$ Bernstein ammetteva che occorresse «considerare improbabile, almeno per un periodo abbastanza lungo, la possibilità di crisi economiche generali» (ivi, p. $117)$.

${ }^{12}$ Poiché «quando una nazione ha raggiunto un assetto politico in cui il diritto della minoranza possidente non è più un serio ostacolo al progresso sociale, in cui i compiti negativi dell'azione politica cedono il passo a quelli positivi, l'appello alla rivoluzione violenta diventa vuota fraseologia. Si può rovesciare un governo, una minoranza privilegiata, non un popolo intero» (ivi, p. 260).

${ }^{13}$ Ivi, 255.

${ }^{14}$ Si veda l'Introduction di N. Urbinati a Liberal Socialism, Princeton University Press, Princeton 1994, p. XLV.

15 «Deterministi e volontaristi, riformisti e rivoluzionari, si contendono aspramente l'eredità del Maestro» (C. Rosselli, Socialismo liberale, cit., p. 29).

${ }^{16}$ Cfr. G. Marramao, Marxismo e revisionismo in Italia - dalla «Critica sociale» al dibattito sul leninismo, De Donato, Bari 1971 e P. Favilli, Storia del marxismo italiano. Dalle origini alla grande guerra, FrancoAngeli, Milano 1996.

${ }^{17}$ C. Rosselli, Socialismo liberale, cit., p. 37.

${ }^{18}$ Ivi, p. 38. L'espressione è ripresa letteralmente da A. Labriola, Del materialismo storico-dilucidazione preliminare (ed. orig. 1896), ora in Id., La concezione materialistica della storia, Laterza, Bari 1971, p. 95. Nel tentativo di conciliare momento oggettivo e momento soggettivo, Labriola affermava che «solo l'amore del paradosso, inseparabile sempre dallo zelo degli appassionati divulgatori di una dottrina 
del determinismo marxista, non priva di ambiguità, non era ancora pienamente condizionata dall'urgenza di rispondere alla crisi del marxismo. La quale si presenta, anche in Italia, nel momento in cui il quadro dei rapporti economici e le condizioni storico-politiche smentiscono le previsioni marxiste, e l'azione del movimento socialista non segue affatto un processo orientato dalla necessità. Di conseguenza, emergono interpretazioni dottrinarie che contraddicono le certezze ortodosse. Rosselli indica nel riformismo e nel sindacalismo rivoluzionario le due vie al revisionismo più rilevanti. Seppur profondamente discordanti, queste due prospettive sono animate da una volontà di «rivalutazione delle forze e dei valori morali», ma palesano entrambe il limite di essere troppo legate alla necessità di «ricavare ad ogni costo dal marxismo elementi di conforto delle proprie tesi pratiche» ${ }^{19}$.

L'analisi si concentra sulla proposta riformista: la critica del riformismo teorico è in effetti la leva per giungere alla critica e al rinnovamento del riformismo politico. Anche in Socialismo liberale è Mondolfo a rappresentare l'interlocutore privilegiato di Rosselli sul tema della interpretazione del marxismo. Viene in tal modo ripresa la polemica già sviluppatasi sulle pagine della "Critica sociale», in cui Rosselli aveva auspicato con spregiudicatezza culturale un distacco netto dalla dottrina marxista, al fine di favorire il superamento della crisi intellettuale del socialismo. Mondolfo allora aveva sottolineato come il momento della volontà politica trovasse non una paralisi, bensì un orientamento, una coscienza, nella teoria del materialismo storico, a cui dava forza il principio del rovesciamento della prassi. Rosselli riconosce in Socialismo liberale l'importanza dello sforzo esegetico mondolfiano e non manca tuttavia di ribadire il limite di una concezione teorica riformista che, pur superando "di fatto" il marxismo, afferma di volere rimanere legata a quest'ultimo, anzi di esserne unica interprete originale. L'intento di Mondolfo sarebbe quello di «estrarre dal marxismo una filosofia del socialismo che si concili pienamente con una

nuova, può avere indotto alcuni nella credenza, che tanto a scriver la storia bastasse di mettere in evidenza il solo momento economico (spesso non accertato ancora, e spesso non accertabile affatto), per poi buttar giù tutto il resto come inutile fardello, di cui gli uomini si fossero caricati a capriccio; come accessorio, insomma, o come semplice bagattella o addirittura come un non - ente». E aggiungeva: "per noi sta, cioè, indiscusso il principio, che non le forme della coscienza determinano l'essere dell'uomo, ma il modo d'essere appunto determina la coscienza (Marx). Ma queste forme della coscienza, come son determinate dalle condizioni di vita, sono anch'esse la storia. Questa non è la sola anatomia economica, ma tutto quello insieme, che cotesta anatomia riveste e ricovre, fino ai riflessi multi colori della fantasia. $\mathrm{O}$, a dirla altrimenti, non c'è fatto della storia che non ripeta la sua origine dalle condizioni della sottostante struttura economica; ma non c'è fatto della storia che non sia preceduto, accompagnato e seguito da determinate forme di coscienza, sia questa superstiziosa o sperimentata, ingenua o riflessa, matura o incongrua, impulsiva o ammaestrata, fantastica o ragionante» (ivi, rispettivamente pp. 69-70 e p. 72).

${ }^{19}$ C. Rosselli, Socialismo liberale, cit., p. 39. 
visione attivistica del processo storico, senza cadere negli eccessi del volontarismo estremo» ${ }^{20}$.

In effetti, come si è accennato, tutta l'opera di Mondolfo è tesa a dimostrare l'intima interdipendenza tra l'uomo e il suo ambiente storicosociale, i quali si trovano in rapporto di azione-reazione, un rapporto per cui l'attività precedente diventa condizione e limite della successiva. La visione del divenire storico delineata attraverso il concetto di rovesciamento della prassi è giudicata convincente, ma paradossalmente non fedele al pensiero di Marx. Nel quale, secondo Rosselli, la dialettica storica si esaurisce nella relazione tra sviluppo tecnico e rapporti sociali, senza spazio per la volontà umana. Se così non fosse, non si spiegherebbe la certezza, o meglio la previsione scientificamente certa, dell'avvento del socialismo. Se Mondolfo, per rivalutare il momento "umano", aveva fatto riferimento alle Glosse su Feuerbach, testo tanto celebre quanto caratterizzato da una concezione dello sviluppo storico poi nettamente superata dal Marx maturo ${ }^{21}$, l'obiettivo teorico della critica rosselliana appare ormai chiaro: il revisionismo, in nome di Marx, è andato oltre $\mathrm{Marx}^{22}$. In mancanza di una stringente necessità storica, senza finalismo, si giunge a una filosofia della prassi che slega il nesso tra marxismo e socialismo. Nesso che, là dove venga pervicacemente riaffermato, è frutto di una visione dogmatica, incapace di sostenere adeguatamente l'azione politica. Non a caso, proprio nella prospettiva di questo squilibrio teorico-pratico, Rosselli motiva la sua critica alla politica socialista precedente l'avvento del fascismo.

\section{Dal revisionismo al socialismo liberale}

Rosselli ribadisce pertanto che l'attaccamento passivo al marxismo ha frenato e danneggiato la strategia politica del partito socialista. Le conseguenze furono l'incomprensione dei compiti nuovi e l'incapacità di raccogliere le sfide politiche che i mutamenti storico-sociali imponevano. Ideologicamente condizionato dai due pilastri del determinismo materialistico e del catastrofismo finalistico, il movimento operaio rimase imbrigliato nelle dispute dottrinarie, diviso tra riformismo economico e rivoluzionarismo verbale. La crisi di fine secolo e le conseguenti

${ }^{20}$ C. Rosselli, Socialismo liberale, cit., p. 42.

${ }^{21}$ In Feuerbach e Marx (1909), Mondolfo sosteneva che «il materialismo storico è - secondo la sola definizione esatta- una concezione critico-pratica. Dalla coscienza critica della realtà sociale alla praxis storica: questo cammino segna il superamento dell'antitesi di volontarismo e fatalismo in un concetto realistico e vivo della necessità storica» (ora in R. Mondolfo, Umanismo di Marx, cit., pp. 69-70). Sul confronto Rosselli-Mondolfo, cfr. l'Introduzione di N. Bobbio a C. Rosselli, Socialismo liberale, cit., pp. XXXIII-XXXVIII.

${ }^{22}$ Sulla critica di Rosselli a Mondolfo, cfr. E. Santarelli, La revisione del marxismo in Italia. Studi di critica storica, Feltrinelli, Milano 1964, p. 162. 
agitazioni operaie avevano condotto il movimento socialista, attraverso un repentino passaggio, «dal bando e dall'ostruzionismo al ministerialismo $»^{23}$. La prassi si era svincolata da qualsiasi velleità rivoluzionaria, per assestarsi sulla rivendicazione parlamentare e sindacale delle categorie sociali di riferimento. Ė questo il punto di volta con cui Rosselli identifica l'inizio della crisi intellettuale ${ }^{24}$. La contraddizione tra teoria e azione divenne sempre più lacerante. A un riformismo ormai istituzionalizzato si giustappose la professione di fede per i dogmi e le certezze messianiche del marxismo. Al gradualismo democratico dei fatti, «la certezza nel rapido inevitabile trapasso $»^{25}$.

Laccusa vale tanto per i massimalisti quanto per i riformisti, a cui Rosselli rivolge maggiormente la sua attenzione, sentendosene parte. Nei primi, il dogmatismo si risolse nell'inconcludente riproposizione di miti logori; nei secondi, esso vincolò la prassi a una pesante cautela determinista. In effetti, i conti con il marxismo non tornavano: il limite riscontrato nella teoria, nel revisionismo, si trasmise anche alla politica, vuoi per un fenomeno di «ipocrisia o di superficialità» (nei rivoluzionari), vuoi per «debolezza $»^{26}$ (nei riformisti). Rosselli è sempre più convinto che l'atteggiamento di chiusura ideologica non abbia pagato. Soprattutto il socialismo gradualista, il solo in grado di dare una svolta teorica e politica, arroccatosi nella difesa delle istanze economiche secondo una prospettiva fiaccamente positivista, non era stato in grado di cogliere l'importanza della battaglia per le riforme politiche in una prospettiva di costruzione democratica. Anche i consensi vennero meno poiché la «nuova generazione tutta idealista, volontarista, pragmatista, non capiva il linguaggio materialistico, positivistico, scientificistico dei vecchi $»^{27}$.

L'automatismo con cui Rosselli collega crisi ideologica e crisi politica risulta ancora una volta schematico e forzato. Se si tiene però conto del contesto storico, della sconfitta di fronte al fascismo e della divisione del fronte socialista, l'impegno con cui è invocata una netta soluzione di continuità rispetto al passato appare maggiormente comprensibile ${ }^{28}$.

${ }^{23}$ C. Rosselli, Socialismo liberale, cit., p. 36.

${ }^{24}$ Nadia Urbinati ha sottolineato che «il divario tra teoria e pratica, ideologia e cultura concreta, era già una realtà antecedentemente alla prima guerra mondiale» (Introduction a Liberal Socialism, cit., p. XLI).

${ }^{25}$ C. Rosselli, Socialismo liberale, cit., p. 36

${ }^{26}$ Ivi, p. 36.

${ }^{27}$ Ivi, p. 48.

${ }^{28}$ Arfè ha rilevato che «le ragioni sono dalla parte di Rosselli quando egli individua nella degradazione della dottrina in ideologia rigida e statica la causa della emarginazione del socialismo dal dibattito delle idee e quando coglie il nesso tra torbido fideismo che attende la palingenesi dal corso delle cose e la passività politica di fronte a eventi e imprevisti non inquadrabili negli antichi schemi», ma che non sono dalla sua parte quando, nell'attaccare il marxismo, non comprende di attaccare «la tradizione socialista nel suo complesso»: tradizione che, sul piano storico 
All'ampia pars destruens succede l'elaborazione della proposta teorica. La tesi di Rosselli è in sostanza la seguente: se il revisionismo ha continuato ad ancorare il socialismo al sistema marxista, cosa che non si giustifica né in teoria né in pratica, bisogna andare oltre il revisionismo stesso e giungere a una concezione liberale del moto socialista. Il tentativo di coniugare socialismo e liberalismo risulta paradossale, se si considera il socialismo nella sua versione marxista. Anzi, in tal caso la contrapposizione è netta. Filosofia della necessità e filosofia della libertà, classismo e individualismo, socializzazione e proprietà privata: nulla è conciliabile tra marxismo e liberalismo. Se, però, l'ideale socialista viene emancipato dagli assunti marxisti, esso si presenta come ansia di giustizia, superamento del privilegio, realizzazione dunque di libertà, non più formale ma sostanziale ${ }^{29}$.

Per giustificare tale mutamento, Rosselli fa appello proprio ai risultati del revisionismo riformista, che, se non ha voluto intaccare il principio d'autorità nei confronti di Marx, ha posto in evidenza tutti gli elementi che ne legittimano il superamento. I revisionisti, infatti, rigettando il determinismo, ponevano nuovamente l'uomo «al centro del processo storico»; non credevano più «alla fatalità dell'avvento, né alla funzione levatrice della violenza e della dittatura $»^{30}$. Rosselli sostiene che, a questo punto, fosse solo necessario il coraggio di giungere alla conclusione più coerente: destituire il marxismo da "tutore ideologico" del movimento socialista. Poiché, privato del determinismo integrale e, quindi, dei suoi esiti finalistici, il marxismo abdicava alla funzione di filosofia della storia. Attraverso una interpretazione deduttiva, egli giunge ad affermare che

[...] la catena del pensiero marxista, ricostruita logicamente - a posteriori - diventa: metodo materialistico, applicazione di esso alla società capitalistica, previsione oggettiva della necessità della soluzione socialista. Il marxista veramente conseguente è dunque socialista per deduzione. Se cade la premessa [...] cade automaticamente la conclusione socialista ${ }^{31}$.

Per liquidare il marxismo Rosselli ricorre a una strategia argomentativa che comprende anche criteri di relativismo storicistico. La dottrina di Marx ha avuto una funzione essenziale nella prima fase della storia del movimento operaio, quando la critica dell'economia politica giustificava

come su quello pratico, permea «la coscienza del singolo militante diventando elemento di coesione, di coerenza, di continuità» (G. Arfè, Carlo Rosselli nella storia del socialismo italiano, in Giustizia e libertà nella lotta antifascista e nella storia d'Italia, cit., p. 35).

${ }^{29}$ Cfr. N. Bobbio, Tradizione ed eredità del liberalsocialismo, in appendice a C. Rosselli, Socialismo liberale, cit., p. 151.

${ }^{30}$ C. Rosselli, Socialismo liberale, cit., p. 28.

${ }^{31}$ Ibid. 
l'emancipazione dalla miseria e il finalismo palingenetico rafforzava la coscienza di classe. Questa funzione si è esaurita nel momento in cui la prassi socialista ha confutato le previsioni specifiche della «teoria del moto e dell'avvento socialista ${ }^{32}$. Nell'articolare il proprio discorso critico, Rosselli attinge a diverse fonti revisionistiche. In particolare, assume interesse il rinvio all'opera di Henry De Man Au delà du marxisme, più volte citata in Socialismo liberale e in cui il socialista belga sosteneva che «il determinismo economico fornisce a un tempo l'esempio della formidabile significazione del marxismo come espressione di una fase primitiva del movimento operaio e del limite della sua efficacia attuale» ${ }^{33}$.

D'altra parte, i princìpi del materialismo storico e della lotta di classe, resi privi delle certezze di un sistema deterministico, rappresentano principi generali di interpretazione storica, validi anche per chi socialista non è, nel senso che «si può essere marxisti senza essere socialisti $»^{34}$. Innegabile la loro importanza come criteri d'indagine e d'orientamento storico, nel «tenere sempre presenti e le forme di produzione, e il grado della tecnica, e i rapporti economici, e la struttura della classe», per considerare così «oltre gli aspetti politici, morali, religiosi, quella che Marx chiamava la struttura economica» ${ }^{35}$. Ma, in effetti, ciò non ha niente a che vedere con la scelta di campo socialista.

La traiettoria interpretativa di Rosselli è qui mutuata dalla lezione crociana. Da Croce deriva il principio della netta separazione teorica tra socialismo e marxismo e la riduzione di quest'ultimo a strumento di interpretazione storica ${ }^{36}$. Il filosofo napoletano aveva difatti affermato che, «spogliato il materialismo storico di ogni sopravvivenza di finalità e di disegni provvidenziali, esso non può dare appoggio né al socialismo né a qualsiasi altro indirizzo pratico della vita $»^{37}$. Sempre Croce aveva negato la validità della dottrina di Marx quale filosofia della storia, vedendo piuttosto nel marxismo un canone orientativo del giudizio storico, il quale "consiglia di rivolgere l'attenzione al cosiddetto sostrato economico delle società, per intendere meglio le loro configurazioni e vicende $»^{38}$.

${ }^{32}$ Ivi, p. 75.

${ }^{33}$ H. De Man, Il superamento del marxismo, Laterza, Bari 1929, p. 183 (ed. orig. 1927). La parabola intellettuale e politica di De Man seguirà un andamento ingloriosamente controverso, incontrando una poco edificante apertura collaborazionista dopo l'invasione tedesca del Belgio all'inizio della seconda guerra mondiale.

${ }^{34}$ C. Rosselli, Socialismo liberale, cit., p. 55. Cfr. l'Introduction di N. Urbinati a Liberal Socialism, cit., p. XLII.

${ }^{35}$ C. Rosselli, Socialismo liberale, cit., p. 74.

${ }^{36}$ Cfr. E. Agazzi, Il giovane Croce e il marxismo, Einaudi, Torino 1962, p. 214.

${ }^{37}$ Croce aggiungeva che «solamente nelle sue determinazioni storiche particolari, nella osservazione che per mezzo di esso sarà possibile fare, si potrà eventualmente trovare un legame tra materialismo storico e socialismo» (B. Croce, Materialismo storico ed economia marxistica, Laterza, Bari 1961, p. 17 (ed. orig. 1900)).

${ }^{38}$ Ivi, p. 81. 
A questa stregua, il marxismo finisce di avere il monopolio dottrinale sul socialismo, divenendo, in ragione di un superamento storicistico, un classico della scienza sociale ${ }^{39}$.

$\mathrm{Si}$ è insistito molto sulla componente idealistica della riflessione rosselliana e sull'ansia di approdo, o meglio di ritorno, a un socialismo etico. È un dato innegabile e tuttavia controbilanciato da un'intenzione tutt'altro che "reazionaria" o romantica. Gli stessi richiami al Risorgimento e a Mazzini si assottigliano in Socialismo liberale fino a divenire del tutto inessenziali: meri tributi di rito. Il superamento del marxismo è legato non a inclinazioni nostalgiche, ma al tentativo di risoluzione pragmatica di una crisi socialista che è sia ideologica - la babele immobilizzante delle mille interpretazioni di Marx - che politica. In quest'ottica, la dottrina tanto vale quanto è utile al fine politico, orientato secondo valori e non secondo dogmi: «ciò che conta, in ultima analisi, è quel tanto di verità, di stimoli, di idealità, che si riesce con l'aiuto della dottrina, a far giungere a una massa $»^{40}$. Lo sbocco liberale del revisionismo è, quindi, non soltanto conclusione logica sotto il profilo teorico, ma anche e soprattutto risposta ai nuovi compiti storici e politici che Rosselli vede assegnati al socialismo. Per un movimento impegnato in una battaglia di libertà - battaglia contingente di contrapposizione al fascismo e di prospettiva per l'edificazione di una democrazia avanzata - non basta il conforto scientifico di una presunta necessità storica né la rievocazione delle pur nobili gesta degli eroi risorgimentali; occorre sollecitare le cause volontaristiche dell'agire politico e metterle al servizio dei compiti che il tempo impone.

Ladesione al socialismo liberale dovrebbe implicare una rivoluzione copernicana rispetto all'assunzione dei principi cardine del socialismo. Lotta di classe, socializzazione, presa del potere, non rappresentano ineluttabili tappe del moto socialista. Possono e devono essere reinterpretate solo come utili mezzi per la crescita morale e il rafforzamento della coscienza civile e politica della classe lavoratrice. Nell'analisi di questo tema - tema essenziale alla comprensione della proposta socialista liberale -, ritorna il riferimento al già menzionato De Man. Andare oltre il marxismo significa tornare alla centralità della politica rispetto all'economia e alla scienza sociale ${ }^{41}$. Rosselli afferma che «non si vuole ammet-

${ }^{39}$ Cfr. F. Sbarberi, L'utopia della libertà eguale, cit., pp. 64-65.

${ }^{40}$ C. Rosselli, Socialismo liberale, cit., p. 78. Bobbio ha notato come «nella filosofia italiana post-positivistica, idealistica e spiritualistica, la critica del marxismo, considerato una filosofia materialistica e deterministica, era quasi obbligata. Ma [...] Rosselli, che pure si era formato nello stesso clima idealistico di tutta la generazione dei primi anni del Novecento, traeva il suo antimarxismo dagli studi di economia e dalla tradizione del pensiero empiristico» (N. Bobbio, Socialismo liberale, «Il Ponte», settembre-ottobre 1989, p. 161).

${ }^{41}$ Rispetto a questo tema la Urbinati ha osservato che «necessità storica e politica sono antitetiche perché la politica necessita di libertà morale e dà spazio alla possibilità» (Introduction a Liberal Socialism, cit., p. XXXV). 
tere il dubbio che possa darsi socializzazione senza che necessariamente ne segua la trasformazione psichica e morale»; e, in relazione alla lotta di classe, che si è «scambiato un principio tattico di indubbio valore pedagogico e strumentale con l'essenza del moto» ${ }^{42}$. Non diversamente De Man aveva sostenuto che

[...] le dottrine socialiste non sono punto il prodotto del fatto che la classe operaia si rende consapevole della propria situazione di classe. All'opposto esse sono una condizione preliminare di codesto risveglio. Il socialismo esisteva già prima del movimento operaio, anzi, prima della classe operaia ${ }^{43}$.

Superamento del socialismo marxista significa attribuire alle scienze sociali il compito di fornire conoscenze per l'orientamento dell'azione, e non di indicare una evoluzione necessaria che escluda l'intervento umano ${ }^{44}$. In tanto il socialismo si fa liberale in quanto non è «né la socializzazione, né il proletariato al potere e neppure la materiale eguaglianza. Il socialismo, colto nel suo aspetto essenziale, è l'attuazione progressiva della idea di libertà e di giustizia tra gli uomini» ${ }^{45}$. L'attuazione è progressiva e non definitiva; e ciò deriva dalla consapevolezza di quanto mutevole e complessa sia la realtà, che non può essere costretta nell'alveo di concetti schematici. Bisogna tenere conto di molteplici fattori in continuo mutamento, «i partiti socialisti concreti, le organizzazioni concrete, le esigenze e gli stimoli effettivi delle masse, la mentalità dei capi politici e sindacalisti» ${ }^{46}$, e spostare l'attenzione dal "radioso avvenire promesso" al perenne presente. Rosselli richiama appunto De Man, che, portando alle estreme conseguenze il motto bernsteiniano "il moto è tutto, il fine è nulla", aveva affermato:

[...] è il movente presente, e non il fine futuro il solo decisivo. Ciò non implica nessuna negazione del fine finale; perché questo, per quel tanto di valore che ha, è rappresentato allo stato di motivo nel movente

${ }^{42}$ C. Rosselli, Socialismo liberale, cit., p. 81.

${ }^{43} \mathrm{E}$ insisteva col sostenere che «l'interesse di classe non spiega tutto. Esso non crea dei moventi etici, ma si limita a dare una forma e una direzione nuova a moventi esistenti insiti nella natura sociale dell'uomo. Questi moventi non possono prolungare la loro azione oltre la durata di una situazione di classe se non in quanto concordino con precetti generali della coscienza umana», (H. De Man, Il superamento del marxismo, cit., p. 127).

${ }^{44}$ Cfr. N. Urbinati, Carlo Rosselli: la democrazia come fede comune, «Il Vieusseux», settembre-dicembre 1994, pp. 34-35.

${ }^{45}$ C. Rosselli, Socialismo liberale, cit., p. 82. Così si era espresso De Man: «il compito dell'educazione socialista mi sembra essere la trasformazione di ideali socialisti in moventi socialisti» (H. De Man, Il superamento del marxismo, cit., p. 259).

${ }^{46}$ C. Rosselli, Socialismo liberale, cit., p. 82. 
attuale; ora esso, in questo caso, non vale se non quello che valgono le azioni che esso determina ${ }^{47}$.

Svanisce così l'idea del socialismo coincidente con la fine della storia e si apre la prospettiva di un socialismo non come «ideale statico e astratto, che potrà un giorno compiutamente realizzarsi», bensì come «ideale limite irraggiungibile che si realizza per quel tanto che riesce a permeare la nostra vita ${ }^{48}$. Occorre aggiungere che Rosselli tende a sottovalutare due insidie. L'esigenza di recuperare il rapporto cruciale tra etica e politica può far perdere di vista i meccanismi concreti che presiedono alla distribuzione del potere tra i soggetti sociali. L'insistenza su moventi che prescindono dall'appartenenza strutturale di classe rischia di depotenziare la capacità di radicamento del socialismo.

\section{Socialismo come realizzazione del liberalismo}

Dopo aver condotto una critica teorico-pratica nei confronti della concezione marxista del socialismo e aver cercato di dimostrare come dal revisionismo si giunga inevitabilmente a una visione liberale del corso della storia, nei tre capitoli finali dell'opera Rosselli tenta di dare un contenuto dottrinale alla formula del socialismo liberale, formula che dovrebbe servire da base per una nuova azione socialista.

Liberalismo e socialismo non si incontrano per giustapposizione, né sono considerate ideologie da sottoporre a composizione sincretica. Non si tratta neanche di ricondurre a sintesi le idee di libertà ed eguaglianza intese filosoficamente; il Socialismo liberale richiama piuttosto un nesso di successione: l'ipotesi del socialismo come movimento che raccoglie, dispiega e tende a realizzare principi originariamente intrinseci al pensiero liberale ${ }^{49}$.

Rosselli aderisce per un verso alla versione idealistica del liberalismo. La libertà è concepita come fine metastorico e perenne dell'uomo. Per un altro verso, l'intangibilità del cuore trascendentale della libertà non ne assicura la salvaguardia e tanto meno il concreto dispiegarsi nella storia e nella vita degli individui. La libertà, sotto questo profilo, non è concepibile come dato di natura, poiché «non si nasce, ma si diventa liberi. E ci si conserva liberi solo mantenendo attiva e vigilante la coscienza della pro-

${ }^{47}$ Ivi, p. 86. Cfr. H. De Man, Il superamento del marxismo, cit., pp. 258-59. Sul rapporto tra Rosselli e De Man, cfr. N. Tranfaglia, Sul socialismo liberale di Carlo Rosselli, in M. Bovero, V. Mura, F. Sbarberi (a cura di), I dilemmi del liberalsocialismo, cit., pp. 88-92 e M. Degl'Innocenti, Socialismo liberale e socialismo europeo, in Id., Carlo Rosselli e il socialismo liberale, cit., pp. 74-77.

${ }^{48}$ C. Rosselli, Socialismo liberale, cit., p. 86.

${ }^{49}$ Cfr. N. Bobbio, Introduzione a Socialismo liberale, cit., pp. XLII- XLIV. 
pria autonomia $»^{50}$. La storia è vista, quindi, come un processo progressivo ma sempre reversibile costellato da tappe significative, che vanno dalla Riforma alla Rivoluzione francese, con conseguente conquista delle libertà religiose, civili, politiche. In questa prospettiva

[...] il socialismo non è che lo sviluppo logico, sino alle sue estreme conseguenze, del principio di libertà. Il socialismo, inteso nel suo significato più sostanziale e giudicato dai risultati - movimento cioè di concreta emancipazione del proletariato - è liberalismo in azione, è libertà che si fa per la povera gente ${ }^{51}$.

Siamo dunque in presenza di un liberalismo che non condivide con il classical liberalism l'idea che la libertà sia un dato assoluto, indistinguibile dall'individuo e bisognoso di una difesa negativa. La libertà è considerata, piuttosto, come un'istanza che si sviluppa dinamicamente, equivale alla ricerca di autonomia sia materiale che spirituale, e si oppone alle diverse forme storiche assunte dal privilegio. In questa chiave, il socialismo può raccogliere il testimone del liberalismo e proseguirne l'opera con l'impegno per la conquista delle libertà economiche ${ }^{52}$. Che non corrispondono alla sacralità del diritto proprietario, ma alla condivisione di condizioni dignitose di vita e a un'equa ripartizione delle opportunità, presupposto per l'effettivo godimento dei diritti civili e politici ${ }^{53}$.

La tesi che pone conquiste liberali e acquisizioni sociali su un piano di complementarietà e accordo reciproco avvicina Rosselli ai sostenitori del cosiddetto liberalismo sociale. Nella sua Storia del liberalismo europeo, Guido De Ruggiero aveva indicato nella Dichiarazione dei diritti la fondazione dell'idea moderna della libertà «che contrasta col formalismo giuridico [...] in quanto mira, più che alla forma, alla sostanza stessa dei diritti». Rivoluzione liberale, rivoluzione democratica e rivoluzione sociale non rappresentano che «l'espansione progressiva di un medesimo spirito [...] spinto fino all'esasperazione del socialismo; esse pertanto rientrano tutte egualmente nella storia della mentalità liberale» ${ }^{54}$. Alla declinazione del linguaggio dei diritti secondo una logica di implicazione e non di distinzione, rinvia l'accento posto da Rosselli sull'importanza delle condizioni materiali di esistenza e sul concetto per cui «la libertà non

${ }^{50}$ Ivi, p. 89.

${ }^{51}$ Ivi, p. 90.

${ }^{52}$ Cfr. N. Bobbio, Attualità del socialismo liberale, in C. Rosselli, Socialismo liberale, cit., p. VII.

${ }^{53}$ Per Nadia Urbinati, Rosselli è «consapevole che il liberalismo ha solo iniziato questo processo - infatti, la condizione di ineguaglianza, ancora propria della maggioranza, trasforma la libertà goduta dalla minoranza in un privilegio» (Introduction a Liberal Socialism, cit., p. XXXIX).

${ }^{54}$ G. De Ruggiero, Storia del liberalismo europeo, Feltrinelli, Milano 1980, p. 71 (ed. orig. 1925). 
accompagnata e sorretta da un minimo di autonomia economica, dalla emancipazione dal morso dei bisogni essenziali, non esiste per l'individuo, è un mero fantasma» $»^{55}$.

Il tema della possibile apertura a obiettivi di giustizia sociale emerge in campo liberale fin dalla seconda metà del XIX secolo. In particolare, la cultura politica inglese esprime riflessioni rilevanti nel campo della conciliazione empirica tra le ragioni della tradizione whig e quelle del lavoro, consolidate attraverso la crescita costante delle organizzazioni sindacali, da cui scaturirà la nascita del Labour Party.

Dal punto di vista filosofico, l'incontro tra liberalismo e socialismo è reso più semplice dalla sostanziale refrattarietà del mondo anglosassone all'influenza marxista ${ }^{56}$. A partire da John Stuart Mill, assume influenza una concezione che attribuisce alla libertà una dimensione sociale oltre che individuale. Nell'utilitarismo riformato di Mill la libertà non è ridotta a licenza di poter agire senza interferenze per soddisfare esigenze egoistiche. Il fine non è il soggetto singolo in senso astratto. L'individuo è storicamente e concretamente collocato in una rete relazionale che dovrebbe assumere valenza strumentale alla crescita e allo sviluppo della società. Il progresso non è un valore misurabile quantitativamente in termini di ricchezza, ma è correlato al continuo allargamento delle opportunità di sviluppo. Nella filosofia sociale di Mill «la condizione migliore per la natura umana è quella per cui, mentre nessuno è povero, nessuno desidera diventare più ricco, né deve temere di essere respinto indietro dagli sforzi compiuti dagli altri per avanzare» ${ }^{57}$.

Sempre nella Storia del liberalismo europeo di De Ruggiero, al nome di Mill è associato quello di Leonard Trelawny Hobhouse, esponente di spicco del New Liberalism inglese ${ }^{58}$ e sostenitore, proprio sulla scia di Mill, di un concetto di libertà fondato "sull'idea della crescenza e dello sviluppo» ${ }^{59}$. È probabile che Rosselli conosca l'opera di Hobhouse, e in particolare il saggio Liberalism, del 1911.

Liberalism ruota intorno all'idea di congiunzione tra libertà e coscienza del bene comune, la cui traduzione pratica implica la rivendicazione

${ }^{55}$ C. Rosselli, Socialismo liberale, cit., p. 91. Cfr. F. Sbarberi, L'utopia della libertà eguale, cit., pp. 61 e 72-73 e P. Costa, Civitas. Storia della cittadinanza in Europa, 4. L'Età dei totalitarismi e delle democrazie, Laterza, Roma-Bari 2001, p. 380.

${ }^{56}$ Cfr. N. Bobbio, Tradizione ed eredità del liberalsocialismo, cit., pp. 151-55.

${ }^{57}$ J.S. Mill, Principi di economia politica, UTET, Torino 1983, 2 voll., p. 1000 (ed. orig. 1848).

${ }^{58}$ Cfr. N. Urbinati, Il liberalismo socialista nella tradizione inglese, cit., pp. 219231.

${ }^{59}$ De Ruggiero sostiene che, nell'elaborazione di Hobhouse, il liberalismo si presenta come "la credenza che la società possa essere costruita su un potere autodirettivo della personalità» e che la libertà diviene "non tanto un diritto dell'individuo quanto una necessità sociale» (G. De Ruggiero, Storia del liberalismo europeo, cit., p. 151). 
di un universale diritto-dovere di self-development. Per Hobhouse, infatti, il «bene comune include ogni individuo, è fondato sulla personalità, e rivendica il massimo spazio per lo sviluppo della personalità di ciascun membro della comunità ${ }^{60}$. Ne discende che la libertà corrisponde a un concetto di relazione, e dovrebbe realizzarsi non in conflitto ma in accordo con l'interesse collettivo. L'affinità con il socialismo di Rosselli consiste in un punto d'incontro sul modo di concepire l'individuo, le cui prerogative non sono salvaguardate secondo la logica del non impedimento, bensì promosse tramite l'arricchimento della capacità di autodeterminazione. A sua volta, l'idea di eguaglianza è sottratta all'identificazione con l'intervento meccanico che limita la libertà e livella i soggetti. L'eguaglianza diviene semmai sinonimo di ampliamento e condivisione della libertà, a partire dal rafforzamento dello statuto di cittadinanza, che si persegue riempiendo di valenza sostanziale il corredo dei diritti, a partire da quelli alla «autonomia e alla intelligenza ${ }^{6}$. In modo simile si era espresso De Ruggiero nella convinzione che «il liberalismo, in quanto universale e diffusa coscienza storica, implica, insieme col sentimento della libertà, l'idea di uguaglianza» ${ }^{62}$.

Il socialismo liberale trova conferma di validità configurando la storia della libertà come storia di opposizione al privilegio. Rosselli riconosce nella borghesia l'agente del progresso liberale nella «lotta contro il dogmatismo della Chiesa e l'assolutismo dei re, contro i privilegi dei nobili e i privilegi del clero, il mondo morto di una produzione immobile e coatta ${ }^{63}$. Questa funzione tuttavia si è andata esaurendo col consolidarsi di un nuovo sistema di potere che comprime le aspettative di un'enorme massa di individui - il quarto stato - e ne impedisce lo sviluppo economico e morale. Con la cristallizzazione dei rapporti economici nel mondo capitalisticoborghese, il liberalismo è stato imprigionato «entro lo schema transeunte di un sistema sociale». Si è così determinata una sconfessione dello spirito liberale, che è per definizione «storicista e relativista, vede nella storia un perpetuo fluire, un eterno divenire e superamento ${ }^{64}$. Nell'analisi di Rosselli riecheggiano di nuovo le argomentazioni di De Ruggiero, che aveva rimarcato il paradosso di un ricorrente scambio delle parti:

[...] si riproduce, a poco a poco, tra proletari e borghesi, la stessa antitesi che s'era già prodotta tra i borghesi e gli aristocratici: sotto le insegne di un universale liberalismo, la borghesia dissimula un privilegio ana-

${ }^{60}$ L.T. Hobhouse, Liberalismo, con un saggio introduttivo di F. Sbarberi, Vallecchi, Firenze 1995, p. 136 (ed. orig. 1911). Nell'Introduzione Sbarberi sottolinea la convergenza ideale tra Hobhouse e Rosselli (ivi, p. 13).

${ }^{61}$ C. Rosselli, Socialismo liberale, cit., p. 70.

${ }^{62}$ G. De Ruggiero, Storia del liberalismo europeo, cit., p. 51.

${ }^{63}$ C. Rosselli, Socialismo liberale, cit., p. 92.

${ }^{64}$ Ivi, p. 93. 
logo a quello che l'aristocrazia ostentava; quindi la lotta dei proletari per smantellare il nuovo privilegio, nella sua apparenza anti-liberale, tenderà in realtà a porre in essere un più largo liberalismo $\mathrm{o}^{65}$.

L'urgenza di correggere le conseguenze distorsive di un sistema economico che produceva privilegi per pochi e creava disuguaglianza di opportunità e impedimento all'autosviluppo per i più, era presente nella letteratura del liberalismo sociale. Mill aveva criticato i risultati prodotti dall'economia di mercato così come si era storicamente sviluppata, con una distribuzione dei frutti del lavoro in proporzione sostanzialmente inversa al lavoro stesso, vale a dire destinando «le quote maggiori a favore di quelli che non hanno mai lavorato del tutto ${ }^{66}$. Aveva pertanto visto favorevolmente la spinta all'emancipazione delle classi subalterne, condizione per l'indipendenza non solo economica, ma soprattutto morale e di giudizio. Dalle lotte sindacali potevano derivare sviluppi da considerare con fiducia. C'era da attendersi «in primo luogo che le classi lavoratrici diverranno anche meno disposte di quanto siano attualmente a lasciarsi guidare e governare [...] ed esse avocheranno a sé il diritto di governare la propria condotta o condizione» ${ }^{67}$. Mill individuava un rapporto di proporzionalità diretta tra la vocazione sociale e l'innalzamento morale degli uomini: giustizia ed eguaglianza si realizzano attraverso «l'associazione, e non l'isolamento degli interessi $»^{68}$. Non va però dimenticata la riserva critica che negli scritti milliani è rivolta alla dimensione sociale, che produce effetti positivi solo nella misura in cui è funzionale all'esaltazione delle individualità e non diviene fonte di appiattimento e omologazione. Da qui i rischi insiti nel prefigurarsi di un sistema socialista, che rimandano proprio all'interrogativo se in tale sistema

[...] l'individualità del carattere potrebbe ancora avere uno spazio; se la pubblica opinione non diventerebbe un gioco tirannico; se l'assoluta dipendenza di ciascuno da tutti, e la sorveglianza di tutti su ciascuno, non finirebbe per ridurre tutti gli uomini ad una tetra uniformità di pensieri, di sentimenti e di azioni ${ }^{69}$.

Rosselli non sembra sottovalutare le ragioni dello scetticismo contenuto nella domanda retorica di Mill. Se è innegabile che la tensione al riscatto delle classi più deboli ha in sé un significato di libertà, affinché l'affrancamento non vada incontro a un'eterogenesi dei fini, occorre che il socialismo, come dottrina e come movimento politico e sindacale, interiorizzi

\footnotetext{
${ }^{65}$ G. De Ruggiero, Storia del liberalismo europeo, cit., p. 48.

${ }^{66}$ J.S. Mill, Principi di economia politica, cit., p. 344.

${ }^{67}$ Ivi, p. 1011.

${ }^{68}$ Ivi, p. 1015.

${ }^{69}$ Ivi, p. 347.
} 
culturalmente princìi e valori liberali. Rosselli non ha dubbi che, nella prassi, la socialdemocrazia europea «si muova verso una forma di rinnovato liberalismo, che riassorbe in sé i motivi di movimenti apparentemen-

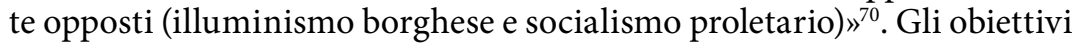
concreti dell'azione politica sono la conquista e la difesa dei diritti civili e politici, e il perseguimento di un maggior benessere economico. Il finalismo messianico el'ideale della società perfetta passano di fatto in secondo piano. Proprio da questa esperienza pratica, il socialismo deve prendere le mosse per un adeguamento dei princìi teorici. Mettere al centro l'uomo, appunto: ciò che significa concepire la società come «un aggregato di individualità [...] mezzo a fine [...] strumento al servizio degli uomini, e non di entità metafisiche, siano esse la Patria, o il Comunismo» ${ }^{71}$. Da una parte la tradizione socialdemocratica è rivalutata, a condizione che non sbocchi in una prospettiva statica e organicistica e che riesca ad accogliere l'idea dello sviluppo individuale come perno di un processo aperto, progressivo, indefinito ${ }^{72}$. Dall'altra parte, la sbrigativa liquidazione del "Comunismo» rivela il disinteresse per le esperienze teoriche più stimolanti del marxismo coevo, a partire dalla riflessione gramsciana. Questa posizione si spiega con il rifiuto di qualsiasi ancoraggio "teologico": il socialismo non è un sistema autosufficiente; è l'opportunità di molteplici condizioni di sviluppo.

Alla ripulsa per le concezioni olistiche corrisponde la rilevanza strategica del principio di autonomia, che da religiosa, civile e politica, deve divenire anche economica. El'autonomia, a sua volta, trova sostanza mediante la partecipazione. Le rivendicazioni per il controllo operaio, le richieste di coinvolgimento nella direzione della produzione, sono innanzitutto lotte di «dignità e responsabilità $»^{73}$. La partecipazione attiva accresce le capacità individuali ed è la premessa indispensabile per la realizzazione del lavoratore «non solo come cittadino ma anche come produttore» ${ }^{74}$.

Il discrimine tra liberalismo sociale e socialismo liberale è inerente al nodo della proprietà privata. Queste due teorie convergono per quanto riguarda l'aspirazione ideale di conciliare libertà e giustizia; differiscono in parte rispetto ai metodi e ai giudizi di natura socio-economica. Il liberalismo apre alla questione sociale allorché concepisce la proprietà

${ }^{70}$ C. Rosselli, Socialismo liberale, cit., p. 88.

${ }^{71}$ Ivi, p. 83.

${ }^{72} \mathrm{Da}$ un punto di vista teorico, liberalismo e socialismo possono incontrarsi solo se il primo viene considerato come teoria liberale dei diritti civili e non come teoria liberista, e il secondo come teoria socialista dei diritti sociali e non come solidarismo olistico. Cfr. M. Bovero, Liberalismo, socialismo, democrazia. Definizioni minime e relazioni possibili, in M. Bovero, V. Mura, F. Sbarberi (a cura di), I dilemmi del liberalsocialismo, cit., pp. 303-320.

${ }^{73}$ C. Rosselli, Socialismo liberale, cit., p. 91.

${ }^{74}$ Ivi, p. 108. Cfr. P. Sylos Labini, Socialismo liberale: gli aspetti economici, «Il Ponte», settembre-ottobre 1989, pp. 169-70. 
non più come diritto soggettivo, bensì funzionale all'interesse generale, poiché «nello stato sociale, in qualunque stato cioè che non sia di totale isolamento, ogni atto che disponga delle cose prodotte non può che aver luogo con il consenso della società, o meglio di coloro che dispongono della forza produttiva della società stessa $»^{75}$. In Hobhouse, il concetto di proprietà funzionale poggia sulla distinzione teorica tra "proprietà d'uso" e "proprietà di potere". La proprietà d'uso riguarda «il controllo di cose, che dà libertà e sicurezza»; la proprietà di potere «il controllo di persone attraverso le cose, che dà potere al padrone» ${ }^{76}$. Il capitalismo tende a creare una divaricazione tra una grande maggioranza, per la quale si riduce la "proprietà d'uso" che è legata alla possibilità di godere dei frutti del proprio lavoro, di cui tutti dovrebbero beneficiare; e una minoranza, nelle cui mani si concentra «l'accumulazione di una vasta massa di "proprietà di potere" ${ }^{77}$, non generata da una attività produttiva. Per Hobhouse, l'interesse sociale coincide con il diritto dell'uomo in primo luogo a una «opportunità di lavoro, in secondo luogo ai frutti del suo lavoro, e infine a ciò che può usare di questi frutti» ${ }^{78}$. L'interesse sociale confligge invece con metodi di accumulazione "che concentrano la ricchezza nelle mani di pochi ${ }^{79}$ e determinano un illegittimo potere di controllo sugli altri. L'obiettivo è dunque contrastare il privilegio tramite interventi che consentano l'ampliamento dell'accesso ai benefici economici e al welfare, garantendo un'azione distributiva tesa a incoraggiare la «suddivisione delle grandi masse di ricchezza ${ }^{80}$ e a potenziare la legislazione sociale.

Il socialismo liberale guarda con maggiore attenzione al lato della produzione, come luogo di una libertà più completa e concreta. Rosselli individua nella partecipazione libera e volontaria alle varie unità associative, di cui si compone la convivenza, la condizione più utile per sostenere il radicamento di un socialismo democratico. Ė il socialismo "dal basso" l'unico in grado di conciliare maggior giustizia sociale e difesa della libertà, evitando le degenerazioni gerarchiche che si annidano nei modelli collettivisti. C'è una vocazione antitotalitaria che induce a indicare i pericoli del socialismo statalistico, accentratore, che fa dello «Stato l'amministratore, il gerente universale, il controllore dei diritti e delle libertà universali » ${ }^{81}$.

${ }^{75}$ J.S. Mill, Principi di economia politica, cit., p. 334.

${ }^{76}$ L.T. Hobhouse, The Historical Evolution of Property, in Id. Sociology and Philosophy, G. Bell and sons LTD, London 1966, p. 89 (ed. orig. 1914).

77 Ivi, p. 98.

${ }^{78}$ Ivi, p. 102.

${ }^{79}$ Ivi, p. 103.

${ }^{80}$ J.S. Mill, Principi di economia politica, cit., p. 345 . Mill aggiunge che, in questa nuova prospettiva, il principio della proprietà individuale non avrebbe nessuna «necessaria connessione con quei mali fisici e sociali che quasi tutti gli scrittori socialisti ritengono invece inseparabili da esso» (Ibid.).

${ }^{81}$ Chiaro il riferimento a quello che verrà definito come il modello del socialismo reale, caratterizzato dai pericoli della «elefantiasi burocratica, della dittatura 
E affiora la percezione che, a prescindere dalle differenti destinazioni proprietarie, capitalismo fordista e capitalismo di stato si somiglino sia nel modo di intendere la gestione dell'impresa, sia sotto il profilo dello status riconosciuto al lavoro. Al confronto il socialismo, così come lo concepisce Rosselli, dovrebbe preservare l'autonomia morale e intellettuale del lavoratore contro «la spaventosa uniformità e la disciplina livellatrice di una produzione standardizzata $»^{82}$.

In Socialismo liberale non è delineato un modello specifico di organizzazione. Emerge un approccio sperimentale, che tenga presente la complessità del mondo economico, e soprattutto eviti di ricondurre a un unico regime le diverse realtà produttive. Nel rispetto di un principio pluralistico, la preferenza è sicuramente accordata a forme di conduzione diversificate, "forme municipali, cooperative, gildiste», senza dimenticare le piccole realtà produttive che, per intrinseche caratteristiche, sfuggono ai criteri di socializzazione integrale (piccola industria, piccola proprietà agraria, mezzadria, artigianato, fittanza). Continua ad esserci un preciso interesse verso il socialismo inglese, e in particolare verso l'opera di Cole ${ }^{83}$, di cui Rosselli apprezza l'ispirazione di fondo, la tensione etica, la critica allo statalismo. Meno convincente viene giudicato il progetto di costituzione gildista, complesso meccanismo di organizzazione istituzionale del sistema economico, secondo una prospettiva statica e armonicistica.

Il socialismo federativo è quello che meglio garantisce la condizione di più ampia autonomia, a cui pensa Rosselli $i^{84}$. In ogni caso, nel tessuto produttivo ci deve essere spazio per la crescita delle capacità individuali, poiché la libertà non può derivare da una «elargizione dall'alto. La liber-

dell'incompetenza, dello schiacciamento d'ogni autonomia e libertà individuale» (C. Rosselli, Socialismo liberale, cit., pp. 98-99). In Liberalism, Hobhouse così si esprimeva: «se dunque esiste veramente un socialismo liberale [...] esso deve chiaramente soddisfare due condizioni. In primo luogo deve essere democratico: deve venire dal basso, non dall'alto [...] deve coinvolgere l'impegno della massa e corrispondere ai suoi desideri profondi, e non a quelli di pochi esseri superiori. In secondo luogo, e per questa stessa ragione, deve fare i conti con l'individuo. Deve dare all'uomo carta bianca nella sua vita personale, per lui così importante; deve essere fondato sulla libertà, ed adoperarsi per lo sviluppo e non per la soppressione della personalità» (L.T. Hobhouse, Liberalism, cit., pp. 169-70).

${ }^{82}$ C. Rosselli, Socialismo liberale, cit., p. 70.

${ }^{83}$ In Storia del liberalismo europeo, anche De Ruggiero, riferendosi alle diverse proposte provenienti dal socialismo inglese, aveva fatto riferimento a «una tendenza gildista, di più spiccata fisionomia liberale, che si fonda sull'azione autonoma $\mathrm{e}$ decentrata dei sindacati» (G. De Ruggiero, Storia del liberalismo europeo, cit., pp. 150-151). Hobhouse, parlando dei socialisti britannici, ammetteva come essi riconoscessero che «il governo popolare non è solo una formula teorica ma una realtà che va difesa e ampliata lottando» (L.T. Hobhouse, Liberalism, cit., p. 209).

${ }^{84}$ Cfr. N. Urbinati, Carlo Rosselli: la democrazia come fede comune, cit., pp. 3940. 
tà è conquista, autoconquista, che si conserva solo col continuo esercizio delle proprie facoltà, delle proprie autonomie» ${ }^{85}$. Rosselli non indica un progetto economico strutturato organicamente; teme modelli rigidi che possano ingabbiare il libero sviluppo delle iniziative autonome. Con prudente duttilità mista a incertezza teorica, sostiene che

[...] solo per grandissime linee si può fissare la meta, anzi una meta, una tappa; che è necessario adeguarsi all'esperienza, tenendo fermi solo alcuni punti saldi di orientamento; perché solo dal moto, dalla esperienza liberamente attuata, scaturiranno le indicazioni per il domani ${ }^{86}$.

\section{La democrazia tra libertà e giustizia possibile}

In Socialismo liberale, il perimetro politico, istituzionale e ideale in cui si può distendere l'azione di sviluppo delle masse è la democrazia. Essa non è identificabile esclusivamente come legge della maggioranza e insieme di norme regolanti il conflitto sociale; rappresenta, altresì, il nucleo di valori che legittimano e garantiscono l'emancipazione politica ed economica degli individui. Nell'inquadrare il tema del rapporto tra socialismo e democrazia, Rosselli distingue due momenti d'analisi. Sotto il profilo teorico, si tratta di risolvere il pregiudizio ideologico antidemocratico, proprio del socialismo marxista, in favore di una piena acquisizione della democrazia come valore. Dal punto di vista storico-politico, occorre invece sottolineare come la crisi del socialismo e l'avvento del fascismo abbiano le proprie radici nella mancata realizzazione in Italia di una democrazia moderna. E muovere da questa premessa per definire l'impegno socialista, in termini negativi, come lotta antifascista, in termini positivi, come costruzione democratica.

Per Rosselli il principio dell'autogoverno, istanza di libertà prodotta dal movimento socialista, ha piena possibilità di realizzazione solo nell'osser-

${ }^{85}$ C. Rosselli, Socialismo liberale, cit., p. 100. Domenico Settembrini ha obiettato che questa visione del socialismo come autoconquista dal basso «per quanto convinta ne fosse l'adozione da parte di Rosselli, contrastava radicalmente con il suo elitismo» (D. Settembrini, Fascisti e azionisti, carissimi amici, «Nuova storia contemporanea», luglio-agosto 1998, p. 59). In realtà, sembrerebbe che Rosselli, pur affermando l'importanza del ruolo ricoperto, ad esempio, dall'élite intellettuale, non abbia una concezione antropologicamente pessimistica della massa. Afferma anzi che «il giudizio pessimistico sulla massa è un giudizio pessimistico sull'uomo» (C. Rosselli, Socialismo liberale, cit., p. 121). Una lettura antielitaria di Rosselli è sostenuta da Sbarberi, il quale sottolinea che «la fiducia nelle capacità di elaborazione politica, di affinamento culturale e di autorganizzazione dei settori di base della società civile induce Rosselli a polemizzare vivacemente anche con le forme ricorrenti di elitismo politico, sia nelle versioni liberali che in quelle di estrema sinistra» (F. Sbarberi, L'utopia della libertà eguale, cit., p. 67).

${ }^{86}$ C. Rosselli, Socialismo liberale, cit., p. 98. 
vanza del «metodo liberale o democratico di lotta politica» ${ }^{87}$. Ciò significa che l'asse socialismo-liberalismo si realizza e si sostanzia all'interno della democrazia ${ }^{88}$. Il rispetto del metodo rinvia infatti alla consapevolezza che progresso sociale e libertà si realizzano attraverso la «libera persuasione del maggior numero ${ }^{89}$ e nell'osservanza dei diritti fondamentali della persona. La democrazia è un patrimonio di civiltà che non ha connotazioni classiste, non è «né borghese né socialista». Rosselli sollecita una adesione «esplicita, integrale, definitiva ${ }^{90}$ alle regole e alle istituzioni democrati$\mathrm{che}^{91}$, riproponendo per tale via anche il problema della conciliazione tra teoria e prassi; in un momento in cui la lotta di libertà è lotta per la democrazia, quest'ultima deve essere considerata un fine $e^{92}$.

La prospettiva politica non può, quindi, essere concepita come conquista del potere finalizzata al rovesciamento delle istituzioni democraticoliberali ${ }^{93}$. L'interiorizzazione della democrazia comporta che, nel caso di una raggiunta maggioranza di governo socialista, dovranno essere osservati i princìi regolativi del metodo democratico, cioè il rispetto dei «diritti delle minoranze dissenzienti» e il «diritto all'opposizione» ${ }^{94}$.

${ }^{87}$ Ivi, p.100.

${ }^{88}$ È stato osservato come Rosselli tenda a identificare liberalismo e democrazia. In effetti, il liberalismo è concepito nella variante storica liberaldemocratica, che è poi quella "vigente" nel momento in cui Rosselli scrive (Cfr. l'Introduzione di Bobbio a C. Rosselli, Socialismo liberale, cit., p. XXIII). Bagnoli afferma che «l'ideologia socialista liberale rappresenta una più ampia coniugazione tra i tre filoni del pensiero moderno - liberale democratico e socialista - poiché se la società dei cittadini ha in se tutte le premesse per divenire una società democratica, è nello sviluppo della democrazia che emerge il presupposto dell'istanza socialista e del suo congiungersi con quella democratica» (P. Bagnoli, La nuova storia, Festina Lente, Firenze 1992, p. 124).

${ }^{89}$ C. Rosselli, Socialismo liberale, p. 100.

${ }^{90}$ Ivi, p. 107. 36.

${ }^{91}$ Cfr. N. Urbinati, Carlo Rosselli: la democrazia come fede comune, cit., pp. 35-

${ }^{92}$ In De Man era presente la stessa preoccupazione, nell'affermazione che «il movimento operaio non è per il marxismo che una semplice lotta di interessi tra le classi, e la democrazia politica non rappresenta per lui che un mezzo per assicurare la vittoria della classe operaia», laddove invece, "anche in senso empirico e storico, la democrazia e il socialismo sono nozioni inseparabili» (H. De Man, Il superamento del marxismo, cit., rispettivamente alle pp. 107 e 113).

${ }^{93}$ Pietro Nenni contesterà la idealizzazione delle istituzioni democratiche presente nel libro di Rosselli, affermando: «non siamo ogni giorno testimoni del fatto che le famose istituzioni liberali e democratiche borghesi sono tutte viziate dalla loro subordinazione inevitabile a quelle che i francesi chiamano "les puissances d'argent"? Non siamo ogni giorno testimoni del valore relativo del suffragio universale, questo tabù dei liberali, di cui i potentati del denaro si giocano le sorti a testa e croce?» (P. Nenni, Il socialismo e la lotta per la libertà, "Avanti!», 17 gennaio 1931, ora in Id., La battaglia socialista contro il fascismo, Mursia, Milano 1977, p. 265).

${ }^{94}$ C. Rosselli, Socialismo liberale, cit., p. 107. 
È viva in Rosselli la considerazione della libertà come valore etico universale, che può essere interpretato storicamente da una classe, ma che non si identifica in questa stessa classe ${ }^{95}$. Con la denuncia dei rischi di involuzione dittatoriale presenti nella concezione della libertà di classe va di pari passo il rilievo che «il problema dei problemi, per tutti i partiti socialisti, diventa ormai quello di darsi un programma che possa soddisfare le necessità di una maggioranza organica della popolazione dei rispettivi paesi» ${ }^{96}$. Del resto, pure da un punto di vista sociologico, la divisione rigida in classi non ha riscontri nella realtà; in particolare "la borghesia non è un blocco uniforme; molto più spesso di quanto non si creda la sua pretesa unità è un sogno di astrattisti ${ }^{97}$. Ancora una volta Rosselli rinvia all'esempio inglese, dove i laburisti, rifiutando l'approccio classista, «non fanno attore il solo proletariato, ma la società, che tutta e in tutte le sue parti si sforzano di trasformare» ${ }^{98}$.

Insomma, il socialismo avanza democraticamente, solo se inteso come processo di crescita culturale che conduce all'assunzione del principio di giustizia come libertà condivisa di un popolo, da parte del popolo. L'interiorizzazione di certi valori non può essere imposta dall'alto; non discende da formule di organizzazione economica o da precetti legisla-

${ }^{95}$ Saragat, in difesa del marxismo, accuserà Rosselli di «concezione formale della libertà». Per Saragat, svincolare la libertà dalla "concezione di un fine cosciente da realizzare», vuol dire definirla come «facoltà di scelta arbitraria sottratta a ogni norma» (G. Saragat, Rosselli e il «socialismo liberale», "Avanti! - L'avvenire del lavoratore», 10 gennaio 1931, ora in Id., Quaranta anni di lotta per la democrazia, Mursia, Milano 1966, p. 180). Evidentemente Saragat riconduce la coscienza del fine alla coscienza di classe, laddove in Rosselli il fine socialista viene ricondotto alla coscienza morale individuale.

${ }^{96}$ C. Rosselli, Socialismo liberale, p. 103.

${ }^{97}$ Ibid. Rosselli sostiene, inoltre, che «alcune frazioni della borghesia esercitano ancora una utile, diciamo anzi, pressoché indispensabile funzione progressista» (ivi, p. 93). L'anticlassismo, che emerge da tali affermazioni, sarà oggetto di un attacco veemente da parte di Palmiro Togliatti, secondo il quale «la esaltazione della "libertà" in generale [...] è impresa reazionaria» che tende a offuscare l'unica azione realmente liberale, quella del «fucile sulla spalla dell'operaio» e della «dittatura esercitata dagli operai per fare a pezzi il mondo capitalistico, per costruire una società socialista» (P. Togliatti, Sul movimento di Giustizia e Libertà, "Lo Stato Operaio», settembre 1931, ora in Id., Opere, III, 1, 1929-1935, Editori Riuniti, Roma 1973, pp. 416-417).

${ }^{98}$ C. Rosselli, Socialismo liberale, cit., p. 85. Giuseppe Bedeschi individua in Rosselli delle forti «oscillazioni», rispetto al problema della lotta di classe. Secondo Bedeschi, Rosselli, in ogni caso, «non riusci a sottrarsi, nonostante le buone intenzioni, a una lettura rigidamente classistica della storia contemporanea: una lettura che egli aveva in comune con i socialisti massimalisti e con i comunisti» (G. Bedeschi, Il «socialismo liberale», utopia sterile e inattuale, «Nuova storia contemporanea», maggio-giugno 1999, p. 25). In realtà, Rosselli non ha una concezione deterministica della lotta di classe. Il suo giudizio non è ideologico, bensì storico, e non si riferisce ad una presunta superiorità etica del proletariato in senso astratto, ma alla valenza liberale da riconoscere alle domande di emancipazione delle masse. 
tivi, ma è, deve essere, il risultato «di persuasione attraverso una lunga catena di esperienze positive» ${ }^{99}$. Mezzo e fine di questo cammino è l'individuo, poiché

[...] la giustizia, la morale, il diritto, la libertà non si realizzano se non per quel tanto che si realizzano nelle singole individualità. Uno Stato giusto non è quello le cui leggi si ispirano a un astratto criterio di giustizia, ma quello in cui i suoi componenti si ispirano nella loro attività concreta a una regola di giustizia ${ }^{100}$.

Per Rosselli, la storia italiana è segnata proprio dall'assenza del processo educativo di una piena e consapevole partecipazione che solo determina l'edificazione della coscienza civile e politica. In Italia tutto questo è mancato, dalla lotta per l'indipendenza, che fu «opera di una minoranza, non passione di popolo», alla costituzione dello Stato nazionale, con la burocrazia piemontese che "avvolse nelle sue spire ordinate ma soffocatrici tutta quanta l'Italia, spegnendo gli estremi aneliti di autonomia $»^{101}$. La dittatura fascista ha trovato terreno fertile in un paese in cui la mancata emancipazione della coscienza nella sfera individuale non ha consentito la «organizzazione della libertà nella sfera sociale, cioè nella costruzione dello Stato e nei rapporti tra i gruppi e le classi $»^{102}$. Il partito socialista, secondo Rosselli, non seppe cogliere l'importanza della lotta per le riforme politiche, premessa per il nascere di una moderna democrazia. Cadde nella trappola del paternalismo giolittiano, seguendo una politica «non realista ma transazionista ${ }^{103}$, per poi sgretolarsi, vittima delle sue stesse divisioni di carattere dogmatico, di fronte al fascismo.

Il nuovo socialismo, di cui Rosselli si fa interprete, va oltre il fine dell'antifascismo come opposizione, per aprire una prospettiva di democrazia a forte contenuto sociale. Ciò comporta un rinnovamento della funzione del movimento socialista. È necessario superare i vincoli ideologici e assecondare tutte le domande di riscatto politico e sociale presenti nel paese, valorizzando il contributo di correnti «particolarmente sensibili ai problemi morali (socialisti mazziniani, etici, cristiani), o ai problemi di autonomia e di forma politica (repubblicani, autonomisti), o ai problemi di libertà e di dignità individuale (socialisti liberali e non pochi sedicenti

${ }^{99}$ Rosselli aggiunge che non si deve avere «troppa fede nelle leggi. Si possono fare tutte le leggi, ma se esse non sanzionano uno stato di fatto in via di affermazione e non riposano già sul costume, si risolvono troppo spesso in conati infruttuosi» (C. Rosselli, Socialismo liberale, cit., p. 109).

${ }^{100}$ Ivi, p. 83.

${ }^{101}$ Ivi, p. 112.

${ }^{102}$ Ivi, p. 111. Cfr. G.B. Furiozzi, Attualità di Carlo Rosselli, «Quaderni del Circolo Rosselli», 1/2000, p. 111.

${ }^{103}$ C. Rosselli, Socialismo liberale, cit., p. 50. 
socialisti anarchici) $»^{104}$. È facile obiettare ancora una volta che in piena dittatura fascista fosse irrealistico sperare di far leva su un'aggregazione così concepita per tentare di rovesciare il regime. In questo modo la riflessione rosselliana verrebbe però fraintesa. Dall'isola di Lipari giunge un monito che guarda al futuro. Il socialismo avrebbe dovuto aprirsi al dibattito pluralista senza inaridirsi in una filosofia ufficiale e - torna il riferimento al laburismo - concepire il partito come «sintesi federativa di tutte le forze che si battono per la causa del lavoro $»^{105}$. È questa, in definitiva, una concezione socialista funzionale all'idea di democrazia partecipata che, nell'ottica ottimista e immaginativa di Rosselli, corrisponde a una proposta di governo rappresentativo di «tutta quanta la classe lavoratrice» ${ }^{106}$.

${ }^{104}$ Ivi, p. 129. Che sul problema delle autonomie e del federalismo Rosselli non abbia ancora maturato idee ben precise è suggerito da C. Malandrino, Socialismo e libertà, FrancoAngeli, Milano, 1990, pp. 84-85.

${ }^{105}$ C. Rosselli, Socialismo liberale, cit., 141.

${ }^{106}$ Ivi, p. 142. 


\section{CONCLUSIONI}

Prima di chiudere, occorre fornire una spiegazione dei motivi che giustificano la scelta di individuare in Socialismo liberale il termine ad quem di questo volume.

L'ultima pagina del libro scritto a Lipari allude all'ipotesi di costituzione di una nuova formazione politica che, nelle intenzioni di Rosselli, avrebbe dovuto ispirarsi alle formulazioni teoriche e al progetto politico contenuti nel libro stesso. Questa ipotesi appena accennata esprime la necessità, che va maturando, di creare un movimento dotato di maggiore autonomia, nel segno di una soluzione di continuità rispetto a programmi e metodi del passato.

Si può quindi affermare che Socialismo liberale rappresenta, allo stesso tempo, il compimento di una ricerca teorica e lo spartiacque verso una nuova esperienza, biografica e intellettuale.

Rosselli tenta, per tutti gli anni '20, di contribuire al rinnovamento del socialismo italiano, nella convinzione che tale obiettivo possa perseguirsi attraverso un'azione dall' interno. La sua visione si scontra però con la rigidità spesso dogmatica delle diverse componenti del movimento socialista. Da qui la decisione di avviare un percorso nuovo. Ė possibile constatare che con la fuga da Lipari e l'esilio in Francia ha inizio una fase sostanzialmente diversa del percorso politico di Rosselli. La creazione di «Giustizia e Libertà», movimento in cui confluiranno diverse anime politiche: socialiste, repubblicane, liberal-democratiche, si colloca al centro del problema storico dell'organizzazione dell'antifascismo in Italia e all'estero.

I motivi della militanza ${ }^{1}$, nel contesto dei rapporti di collaborazione e conflittualità tra le forze della Concentrazione antifascista, e la ricerca di una soluzione rivoluzionaria per il rovesciamento del regime di Mussolini, incideranno notevolmente sulla riflessione rosselliana. L'ipotesi di unità socialista, legata fino ad allora al progetto teorico del socialismo liberale,

${ }^{1}$ In una lettera da Parigi del 7 febbraio 1930, Rosselli scriverà a Luigi Sturzo: «Difficile...conciliare l'attività pratica con la teoretica», aggiungendo: "quest'ultimo mese è stato il mese più pragmatistico della mia vita» (G. Grasso (a cura di), Luigi Sturzo e i Rosselli tra Londra, Parigi e New York: carteggio (1929-1945); prefazione di G. De Rosa, Rubbettino, Soveria Mannelli 2003, p. 49). 
sarà condizionata dalle scelte di strategia politica. In quest'ottica si porrà il difficile e contraddittorio problema dell'alleanza con i comunisti, anche alla luce dei cambiamenti dello scenario internazionale. In un quadro tumultuoso, Rosselli darà fondo alla sua vena volontaristica, impegnandosi strenuamente nell'azione di lotta antifascista, come dimostra la decisione di partecipare alla guerra civile spagnola. L'uccisione in Francia non gli consentirà di proseguire l'impegno nell'ambito della resistenza $\mathrm{e}$, cosa che qui più preme, di continuare ad approfondire la ricerca teorica, nel contesto rinnovato della fine della guerra e della fondazione della repubblica democratica.

Ringrazio Francesca, che ha letto, commentato e corretto ogni parte di questo lavoro: il suo aiuto è stato indispensabile. E ringrazio Alberto, per l'impagabile disponibilità. 
Acerbo G. 41

Agazzi E. 89

Albertini L. 42

Albertoni E.A. 32

Angelini G. 19

Ansaldo G. 62

Aquarone A. 39

Artom E. 25

Ascoli M. 20

Bagnoli P. 1, 12, 15, 22, 32, 40, 56, 71, 75-76, 101

Baratono A. 77

Basso L. 78

Bauer R. 29, 45-46

Becchio G. 68

Bechelloni A. 40

Bedeschi G. 1, 102

Beilharz P. 63, 67, 70

Belardelli G. 8

Benedetto XV 14

Berneri C. 29

Bernstein E. 20, 83-84

Bianchi G. 68

Bignami E. 19

Bobbio N. 37, 53, 81, 83, 86, 88, 90, 92-94, 101

Bonomi I. 22

Borsa M. 62

Bovero M. 43, 48, 58, 92, 97

Bravo G.M. 68

Cabiati A. 28, 68
Calabi D. 6

Calamandrei P. 8, 19, 27

Calloni M 6-7

Campos Boralevi L. 8

Candeloro G. 30

Caramella S. 78

Carini C. 19

Casucci C. 11

Cavaglion A. 7

Cavallari G. 22

Cedroni L. 7

Chiarini R. 32

Ciuffoletti Z. 5, 12, 27, 39, 44, 80

Coduri A. 62

Cofrancesco D. 3, 19

Cole G.D.H. 25, 58-59, 62, 65-68, 70-71, 99

Colombo A. 71

Corradini E. 9

Costa P. 94

Crespi A. 62

Crispi F. 35, 47

Croce B. $12,32,89$

D'Orsi A. 10

De Felice R. 39

De Man H. 89-92, 101

De Rosa G. 105

De Ruggiero G. 44, 62, 93-96, 99

De Sanctis A. 60

De Stefani A. 30

Degl'Innocenti M. 3, 26, 65, 71, 92

Carmelo Calabrò, Liberalismo, democrazia, socialismo. L'itinerario di Carlo Rosselli, ISBN 978-88-6453-084-0 (print), ISBN 978-88-6453-086-4 (online)

(C) 2009 Firenze University Press 
Del Corno N. 28

Della Volta R. 19

Di Vito L. 81

Dorso G. 32

Einaudi L. 2, 27-29, 33-37, 42, 46, 68

Faucci R. 36

Favilli P. 22, 84

Feuerbach L. 56, 86

Finzi E. 27

Fiori G. 5, 10, 17-18

Flores D'Arcais P. 32

Foa V. 14

Foote G. 64-65

Francovich C. 27

Franzinelli M. 6

Frontali G. 27

Furiozzi G.B. 22, 103

Galasso G. 36

Garin E. 9

Garosci A. 5, 18, 28, 80-81

Gentile E. 9-11

Gervasoni M. 47

Gialdroni M. 81

Giolitti G. 9, 17, 29, 74, 77, 79

Giordani F.P. 62

Gobetti P. 15, 22, 27, 29-30, 32, $34,37,41-44,46-48,51,61-$ $62,71-72$

Golzio F. 12

Gramsci A. 14-15, 49-51

Grassi Orsini F. 39

Grasso G. 105

Graziadei A. 22

Guerra A. 12, 15, 80

Guidi M.E.L. 29, 68

Hilferding R. 25

Hobhouse L.T. 58, 60, 94-95, 98-99

Hobson J.A. 58
Isnenghi M. 17

Jahier P. 27, 62

Jannaccone P. 27

Kuliscioff A. 49

Labriola Antonio 20, 29-30, 50, 72-73, 84

Labriola Arturo 20, 29-30, 72

Lenin (Uljanov V.I.) 4, 39

Leone E. 20

Levi A. 8, 12, 14, 18, 46

Levi C. 42

Levi N. 59, 62, 80

Levi Sullam S. 8

Limentani L. 62

Limone G. 27

Loria A. 27

Lussu E. 81

Luzzato G. 62

MacDonald J. R. 58

Magid H.M. 70

Malandrino C. 68,104

Marucco D. 62

Marx K. 15, 22, 32, 39, 50-53, 56, 58, 60-61, 77-79, 82-86, 88-90

Mastellone S. 58-61, 63, 70, 73, 76, 79

Matteotti G. 18, 28, 39, 46, 50

Mazzini G. 7, 90

Merli S. 71

Michelini L. 29, 68

Mill J.S. 58, 94, 96, 98

Missiroli M. 42

Modigliani G.E. 50

Mondolfo U.G. 12

Mondolfo R. 12, 14-15, 18, 50-51, 53-58, 78, 85-86

Monti A. 42

Morandi R. 77-78

Mura V. 43, 48, 58, 92, 97 
Mussolini B. 28, 39, 41, 47, 49, 71, Salandra A. 41-42 81,105

Salvadori M. 17, 49, 75

Salvemini G. $8-9,11-12,16-18,27-$

Nenni P. $8,12,71-73,76-78,80$, 101

Neri Serneri S. 73

Niccoli A. 27

Nitti F.F. 81

Nitti F.S. 17,68

Orvieto L. 7

Pagano G. 35

Palazzolo C. 62

Papini G. 9

Pareto V. 52

Parri F. 81

Pastori P. 22

Pertini S. 81

Pincherle G. 5-7

Prato G. 34, 69

Prezzolini G. 9, 11, 42-43

Procacci G. 11

Pugliese S.G. 5, 18

Quagliariello G. 39

Rapone L. 80

Rati A. 14

Revelli M. 48, 61

Ripepe E. 32

Rockow L. 63-64, 66-67

Rosselli Aldo 6, 10

Rosselli Amelia 5-10

Rossi E. 17, 27

Rosselli G. 7

Rosselli J. 6

Rosselli Maria 5, 20

Rosselli Marion 5

Rosselli N. 5-8, 10-11, 17, 27, 40, 62,75

Rosselli P. 7 $29,42,46,62,71,76$

Santarelli E. 86

Saragat G. 77, 102

Sbarberi F. 1, 43, 48, 58, 60, 90, 92, 94-95, 97, 100

Schiavi A. 59

Sereni E. 5, 59

Settembrini D. 21, 78, 100

Sighele S. 9

Signori E. 71

Sorel G. 20, 22-23

Spriano P. 34-35

Sraffa P. 59

Sternhell Z. 23

Sturzo L. 105

Suppa S. 43

Taiuti A. 20

Terracini U. 49

Togliatti P. 49, 102

Tranfaglia N. 5, 15, 17-18, 61-63, 71, 76-77, 79, 81, 92

Treves C. 28-29, 38, 50-51, 61, 74-75

Treves R. 51

Turati F. 8, 12, 14, 16-19, 22, 28-29, $38,47,49-50,81$

Urbinati N. 58, 79, 84, 87, 89-91, 9394, 99, 101

Valiani L. 5

Vander F. 15, 49

Ventrone A. 10

Vidotto V. 80

Viroli M. 6

Visciola S. 27

Vivanti C. 6

Vivarelli R. 12, 16

Sabbatucci G. 41,80

Webb B. $25,58,62-64,67$ 
Webb S. 25, 58, 62-64, 67

Winckelmann J.J. 12

Wilson T.W. 13-15

Wohl R. 13
Zabban Giorgina 7

Zabban Giulio 5, 7, 17

Zaniboni T. 73

Zucàro D. 71-73, 76-78, 80 
1. Brunetto Chiarelli, Renzo Bigazzi, Luca Sineo (a cura di), Alia: Antropologia di una comunità dell'entroterra siciliano

2. Vincenzo Cavaliere, Dario Rosini, $D a$ amministratore a manager. Il dirigente pubblico nella gestione del personale: esperienze a confronto

3. Carlo Biagini, Information technology ed automazione del progetto

4. Cosimo Chiarelli, Walter Pasini (a cura di), Paolo Mantegazza. Medico, antropologo, viaggiatore

5. Luca Solari, Topics in Fluvial and Lagoon Morphodynamics

6. Salvatore Cesario, Chiara Fredianelli, Alessandro Remorini, Un pacchetto evidence based di tecniche cognitivocomportamentali sui generis

7. Marco Masseti, Uomini e (non solo) topi. Gli animali domestici e la fauna antropocora

8. Simone Margherini (a cura di), BIL Bibliografia Informatizzata Leopardiana 1815-1999: manuale d'uso ver. 1.0

9. Paolo Puma, Disegno dell'architettura. Appunti per la didattica

10. Antonio Calvani (a cura di), Innovazione tecnologica e cambiamento dell'università. Verso l'università virtuale

11. Leonardo Casini, Enrico Marone, Silvio Menghini, La riforma della Politica Agricola Comunitaria e la filiera olivicolo-olearia italiana

12. Salvatore Cesario, L'ultima a dover morire è la speranza. Tentativi di narrativa autobiografica e di "autobiografia assistita"

13. Alessandro Bertirotti, L'uomo, il suono e la musica

14. Maria Antonietta Rovida, Palazzi senesi tra '600 e '700. Modelli abitativi e architettura tra tradizione e innovazione

15. Simone Guercini, Roberto Pio- van, Schemi di negoziato e tecniche di comunicazione per il tessile e abbigliamento

16. Antonio Calvani, Technological innovation and change in the university. Moving towards the Virtual University

17. Paolo Emilio Pecorella, Tell Barri/Kahat: la campagna del 2000. Relazione preliminare

18. Marta Chevanne, Appunti di Patologia Generale. Corso di laurea in Tecniche di Radiologia Medica per Immagini e Radioterapia

19. Paolo Ventura, Città e stazione ferroviaria

20. Nicola Spinosi, Critica sociale e individuazione

21. Roberto Ventura (a cura di), Dalla misurazione dei servizi alla customer satisfaction

22. Dimitra Babalis (a cura di), Ecological Design for an Effective Urban Regeneration

23. Massimo Papini, Debora Tringali (a cura di), Il pupazzo di garza. L'esperienza della malattia potenzialmente mortale nei bambini e negli adolescenti

24. Manlio Marchetta, La progettazione della città portuale. Sperimentazioni didattiche per una nuova Livorno

25. Fabrizio F.V. Arrigoni, Note su progetto e metropoli

26. Leonardo Casini, Enrico Marone, Silvio Menghini, OCM seminativi: tendenze evolutive e assetto territoriale

27. Pecorella Paolo Emilio, Raffaella Pierobon Benoit, Tell Barri/Kahat: la campagna del 2001. Relazione preliminare

28. Nicola Spinosi, Wir Kinder. La questione del potere delle relazione adulti/ bambini

29. Stefano Cordero di Montezemolo, I profili finanziari delle società vinicole 
30. Luca Bagnoli, Maurizio Catalano, Il bilancio sociale degli enti non profit: esperienze toscane

31. Elena Rotelli, Il capitolo della cattedrale di Firenze dalle origini al XV secolo

32. Leonardo Trisciuzzi, Barbara Sandrucci, Tamara Zappaterra, Il recupero del sé attraverso l'autobiografia

33. Nicola Spinosi, Invito alla psicologia sociale

34. Raffaele Moschillo, Laboratorio di disegno. Esercitazioni guidate al disegno di arredo

35. Niccolò Bellanca, Le emergenze umanitarie complesse. Un'introduzione

36. Giovanni Allegretti, Porto Alegre una biografia territoriale. Ricercando la qualità urbana a partire dal patrimonio sociale

37. Riccardo Passeri, Leonardo Quagliotti, Christian Simoni, Procedure concorsuali e governo dell'impresa artigiana in Toscana

38. Nicola Spinosi, Un soffitto viola. Psicoterapia, formazione, autobiografia

39. Tommaso Urso, Una biblioteca in divenire. La biblioteca della Facoltà di Lettere dalla penna all'elaboratore. Seconda edizione rivista e accresciuta

40. Paolo Emilio Pecorella, Raffaella Pierobon Benoit, Tell Barri/Kahat: la campagna del 2002. Relazione preliminare

41. Antonio Pellicanò, $\mathrm{Da}$ Galileo Galilei a Cosimo Noferi: verso una nuova scienza. Un inedito trattato galileiano di architettura nella Firenze del 1650

42. Aldo Burresi (a cura di), Il marketing della moda. Temi emergenti nel tessile-abbigliamento

43. Curzio Cipriani, Appunti di museologia naturalistica

44. Fabrizio F.V. Arrigoni, Incipit. Esercizi di composizione architettonica

45. Roberta Gentile, Stefano Mancuso, Silvia Martelli, Simona Rizzitelli, Il Giardino di Villa Corsini a Mezzomonte. Descrizione dello stato di fatto e proposta di restauro conservativo

46. Arnaldo Nesti, Alba Scarpellini (a cura di), Mondo democristiano, mon- do cattolico nel secondo Novecento italiano

47. Stefano Alessandri, Sintesi e discussioni su temi di chimica generale

48. Gianni Galeota (a cura di), Traslocare, riaggregare, rifondare. Il caso della Biblioteca di Scienze Sociali dell'Università di Firenze

49. Gianni Cavallina, Nuove città antichi segni. Tre esperienze didattiche

50. Bruno Zanoni, Tecnologia alimentare 1. La classe delle operazioni unitarie di disidratazione per la conservazione dei prodotti alimentari

51. Gianfranco Martiello, La tutela penale del capitale sociale nelle società per azioni

52. Salvatore Cingari (a cura di), Cultura democratica e istituzioni rappresentative. Due esempi a confronto: Italia e Romania

53. Laura Leonardi (a cura di), Il distretto delle donne

54. Cristina Delogu (a cura di), Tecnologia per il web learning. Realtà e scenari

55. Luca Bagnoli (a cura di), La lettura dei bilanci delle Organizzazioni di Volontariato toscane nel biennio 2004-2005

56. Lorenzo Grifone Baglioni (a cura di), Una generazione che cambia. Civismo, solidarietà e nuove incertezze dei giovani della provincia di Firenze

57. Monica Bolognesi, Laura Donati, Gabriella Granatiero, Acque e territorio. Progetti e regole per la qualità dell'abitare

58. Carlo Natali, Daniela Poli (a cura di), Città e territori da vivere oggi e domani. Il contributo scientifico delle tesi di laurea

59. Riccardo Passeri, Valutazioni imprenditoriali per la successione nell'impresa familiare

60. Brunetto Chiarelli, Alberto Simonetta, Storia dei musei naturalistici fiorentini

61. Gianfranco Bettin Lattes, Marco Bontempi (a cura di), Generazione Erasmus? L'identità europea tra vissuto e istituzioni 
62. Paolo Emilio Pecorella, Raffaella Pierobon Benoit, Tell Barri / Kahat. La campagna del 2003

63. Fabrizio F.V. Arrigoni, Il cervello delle passioni. Dieci tesi di Adolfo Natalini

64. Saverio Pisaniello, Esistenza minima. Stanze, spazî della mente, reliquiario

65. Maria Antonietta Rovida (a cura di), Fonti per la storia dell'architettura, della città, del territorio

66. Ornella De Zordo, Saggi di anglistica e americanistica. Temi e prospettive di ricerca

67. Chiara Favilli, Maria Paola Monaco, Materiali per lo studio del diritto antidiscriminatorio

68. Paolo Emilio Pecorella, Raffaella Pierobon Benoit, Tell Barri / Kahat. La campagna del 2004

69. Emanuela Caldognetto Magno, Federica Cavicchio, Aspetti emotivi e relazionali nell'e-learning

70. Marco Masseti, Uomini e (non solo) topi ( $2^{\mathrm{a}}$ edizione)

71. Giovanni Nerli, Marco Pierini, Costruzione di macchine

72. Lorenzo Viviani, L'Europa dei partiti. Per una sociologia dei partiti politici nel processo di integrazione europea

73 Teresa Crespellani, Terremoto e ricerca. Un percorso scientifico condiviso per la caratterizzazione del comportamento sismico di alcuni depositi italiani

74 Fabrizio F.V. Arrigoni, Cava. Architettura in "ars marmoris"

75. Ernesto Tavoletti, Higher Education and Local Economic Development
76. Carmelo Calabrò, Liberalismo, democrazia, socialismo. L'itinerario di Carlo Rosselli (1917-1930)

77. Luca Bagnoli, Massimo Cini (a cura di), La cooperazione sociale nell'area metropolitana fiorentina. Una lettura dei bilanci d'esercizio delle cooperative sociali di Firenze, Pistoia e Prato nel quadriennio 2004-2007

78. Lamberto Ippolito, La villa del Novecento

79. Cosimo Di Bari, A passo di critica. Il modello di Media Education nell'opera di Umberto Eco

80. Leonardo Chiesi (a cura di), Identità sociale e territorio. Il Montalbano

81. Piero Degl'Innocenti, Cinquant'anni, cento chiese. L'edilizia di culto nelle diocesi di Firenze, Prato e Fiesole (1946-2000)

82. Giancarlo Paba, Anna Lisa Pecoriello, Camilla Perrone, Francesca Rispoli, Partecipazione in Toscana: interpretazioni e racconti

83. Alberto Magnaghi, Sara Giacomozzi (a cura di), Un fiume per il territorio. Indirizzi progettuali per il parco fluviale del Valdarno empolese

84. Dino Costantini (a cura di), Multiculturalismo alla francese?

85. Alessandro Viviani (a cura di), Firms and System Competitiveness in Italy

86. Paolo Fabiani, The Philosophy of the Imagination in Vico and Malebranche

87. Carmelo Calabrò, Liberalismo, democrazia, socialismo. L'itinerario di Carlo Rosselli 
PRISMA S.p.A.

Via Marziale, $\mathrm{n} 13$

04023 Formia (LT) 